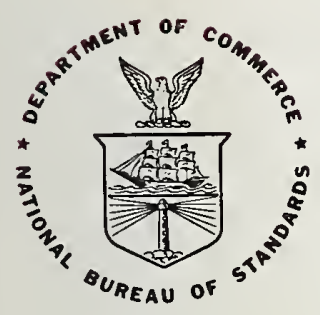

NBS TECHNICAL NOTE 1189

U.S. DEPARTMENT OF COMMERCE/National Bureau of Standards

\title{
A Thermodynamic Surface for the Critical Region of Ethylene
}




\section{NATIONAL BUREAU OF STANDARDS}

The National Bureau of Standards' was established by an act of Congress on March 3, 1901. The Bureau's overall goal is to strengthen and advance the Nation's science and technology and facilitate their effective application for public benefit. To this end, the Bureau conducts research and provides: (1) a basis for the Nation's physical measurement system, (2) scientific and technological services for industry and government, (3) a technical basis for equity in trade, and (4) technical services to promote public safety. The Bureau's technical work is performed by the National Measurement Laboratory, the National Engineering Laboratory, and the Institute for Computer Sciences and Technology.

THE NATIONAL MEASUREMENT LABORATORY provides the national system of physical and chemical and materials measurement; coordinates the system with measurement systems of other nations and furnishes essential services leading to accurate and uniform physical and chemical measurement throughout the Nation's scientific community, industry, and commerce; conducts materials research leading to improved methods of measurement, standards, and data on the properties of materials needed by industry, commerce, educational institutions, and Government; provides advisory and research services to other Government agencies; develops, produces, and distributes Standard Reference Materials; and provides calibration services. The Laboratory consists of the following centers:

\section{Absolute Physical Quantities ${ }^{2}$ - Radiation Research - Chemical Physics - Analytical Chemistry - Materials Science}

THE NATIONAL ENGINEERING LABORATORY provides technology and technical services to the public and private sectors to address national needs and to solve national problems; conducts research in engineering and applied science in support of these efforts; builds and maintains competence in the necessary disciplines required to carry out this research and technical service; develops engineering data and measurement capabilities; provides engineering measurement traceability services; develops test methods and proposes engineering standards and code changes; develops and proposes new engineering practices; and develops and improves mechanisms to transfer results of its research to the ultimate user. The Laboratory consists of the following centers:

Applied Mathematics - Electronics and Electrical Engineering ${ }^{2}$ - Manufacturing Engineering - Building Technology - Fire Research - Chemical Engineering ${ }^{2}$

THE INSTITUTE FOR COMPUTER SCIENCES AND TECHNOLOGY conducts research and provides scientific and technical services to aid Federal agencies in the selection, acquisition, application, and use of computer technology to improve effectiveness and economy in Government operations in accordance with Public Law 89-306 (40 U.S.C. 759), relevant Executive Orders, and other directives; carries out this mission by managing the Federal Information Processing Standards Program, developing Federal ADP standards guidelines, and managing Federal participation in ADP voluntary standardization activities; provides scientific and technological advisory services and assistance to Federal agencies; and provides the technical foundation for computer-related policies of the Federal Government. The Institute consists of the following centers:

Programming Science and Technology - Computer Systems Engineering.

'Headquarters and Laboratories at Gaithersburg, MD, unless otherwise noted; mailing address Washington, DC 20234.

${ }^{2}$ Some divisions within the center are located at Boulder, CO 80303. 


\section{A Thermodynamic Surface for the Critical Region of Ethylene}

J.M.H. Levelt Sengers', G.A. Olchowy'2, B. Kamgar-Parsi', and J.V. Sengers ${ }^{1,2}$

1. Thermophysics Division Center for Chemical Engineering

National Engineering Laboratory

National Bureau of Standards

Washington, DC 20234

2. Institute for Physical Science and Technology

University of Maryland

College Park, MD 20742

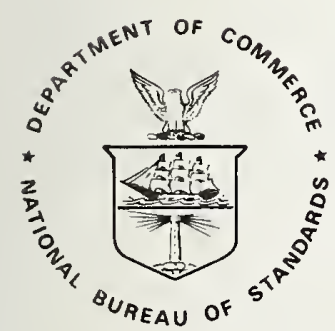

U.S. DEPARTMENT OF COMMERCE, Malcolm Baldrige, Secretary NATIONAL BUREAU OF STANDARDS, Ernest Ambler, Director Issued May 1984 


\section{National Bureau of Standards Technical Note 1189}

Natt. Bur. Stand. (U.S.), Tech. Note 1189, 95 pages (May 1984) CODEN: NBTNAE

\section{U.S. Government Printing Office}

\section{Washington: 1984}


Table of Contents

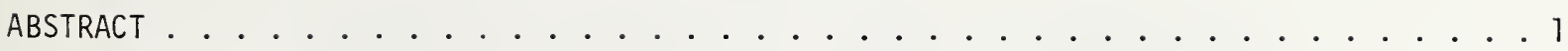

1. Introduction . . . . . . . . . . . . . . . . . 2

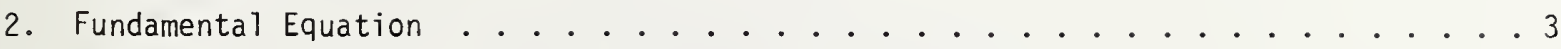

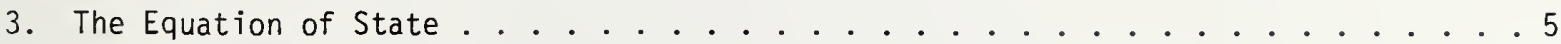

4. Critical Parameters ..................... 6

5. Coexistence Properties. . . . . . . . . . . . . . . . . . . 7

6. Speed of Sound ....................... . . 7

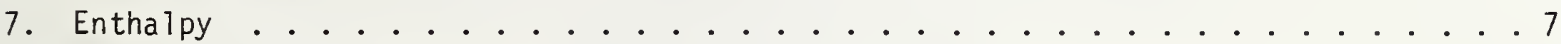

8. Other Formulations ..................... . . . 8

9. The Effect of Pressure and Temperature Errors on Density . . . . . . . . . 9

10. The Effect of Impurity on Density . . . . . . . . . . . . . . . 10

11. Tabies of Thermodynamic Properties . . . . . . . . . . . . . . . . 11

12. References. . . . . . . . . . . . . . . . . . . 11

APPENDIX A Revised and Extended Scaling Equations for the

Thermodynamic Properties of Fluids. . . . . . . . . . . . . . 14

APPENDIX B Comparison with Experiment . . . . . . . . . . . . . . . 20

APPENDIX C Tables of Thermodynamic Properties . . . . . . . . . . . . . . . 33

APPENDIX 0 Listing of Computer Program for Table Generation . . . . . . . . . . 58

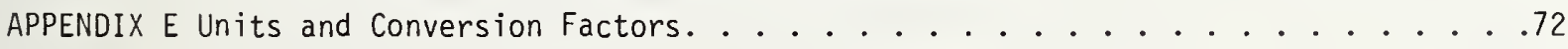

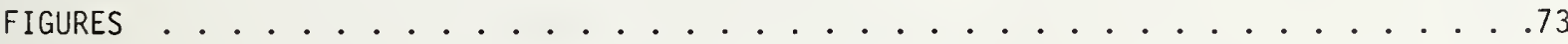



A Thermodynamic Surface for the Critical Region of Ethylene?

by

\author{
J.M.H. Levelt Sengers ${ }^{a}$, G.A. O1chowy ${ }^{b}$, B. Kamgar-Parsi $i^{b}$, \\ and J.V. Sengers ${ }^{a, b}$ \\ a. Thermophysics Division \\ Center for Chemical Engineering \\ National Engineering Laboratory \\ National Bureau of Standards \\ Washington, DC 20234 \\ b. Institute for Physical Science and Technology \\ University of Maryland \\ College Park, MD 20742
}

Tables are presented of thermodynamic properties of ethylene in the range 279-300 $\mathrm{K}$ in temperature, $5.75-10.5 \mathrm{~mol} / \mathrm{dm}^{3}$ in density, which range includes the critical point. The tables presented here are based on the critical-point scaling laws and incorporate the critical anomalies as presently known from renormalization-group theory. The tables complement the formulation of the equation of state of fluid ethylene by McCarty and Jacobsen (NBS Tech. Note 1045, 1981) which does not claim accuracy near the critical point. The predictions of the present formulation are compared with four sets of recent PVT data, and with speed-of-sound and enthalpy data. Tables are presented of pressure, energy, enthalpy, entropy, specific heats and speed of sound as function of temperature along finely-spaced isochores. The computer program required for table generation is included. Even if the surface were perfect, the reliability of densities calculated at experimental pressures and temperatures of limited accuracy declines rapidly as the critical point is approached. Contour plots in P-T space are presented of regions to be avoided in custody transfer for given uncertainties in pressure, temperature and sample composition.

Key words: critical region; custody transfer; density; enthalpy; ethylene; equation of state; impurity; scaling laws, specific heat; speed of sound; supercritical extraction; thermodynamic properties.

\footnotetext{
This work was part of a Joint Industry - Government project under the sponsorship of Celanese Chemical Company, Cities Service Company, Continental 0il Company, Gulf Research and Development, Mobil Chemical Company, Monsanto Polymers and Petrochemicals Company, Phillips Chemical Company, Union Carbide Corp. and the National Bureau of Standards.
} 


\section{Introduction}

A formulation is presented of the thermodynamic behavior of ethylene in a region around the critical point extending from 5.75 to $10.5 \mathrm{~mol} / \mathrm{dm}^{3}$ in density and from 279 to $300 \mathrm{~K}$ in temperature. A correct formulation of this near-critical region is more urgent for ethylene than for most other fluids, because in the climatic conditions of the United States the fluid transported in pipes can assume states located in this region. The present formulation will also be useful as a characterization of ethylene as a supercritical solvent. It is well recognized that most engineering equations of state are deficient in representing near-critical states [1-4]. Our formulation of the thermodynamic behavior of ethylene derives from a thermodynamic potential which obeys the critical-point scaling laws; it contains a first correction to scaling and also a revision to scaling designed to accommodate the lack of symmetry of the gas-liquid phase transition. The adjustable parameters in the potential were obtained by a fit to the PVT data of Hastings et al. [5] and to the speed-of-sound data of Gammon [6]. The advantage of our formulation is that the critical anomalies that are common to all fluids are properly incorporated. For instance, the flatness of the critical isotherm, which is hard to describe by analytic equations of state, is built into our potential. Its strength is particularly striking when it comes to presenting the specific heat at constant volume, $c_{V}$, and the speed of sound, $c_{s}$. The weak divergence of $C_{V}$ cannot be described by analytic free energy surfaces. This divergence, however, causes the speed of sound to approach the value zero at the critical point, a feature properly incorporated in our potential. The excellent speed-of-sound data of Gammon therefore form a crucial test for the adequacy of our thermodynamic surface. We stress that, by the use of so-called parametric variables, we have closed-form expressions for all thermodynamic properties.

We will describe how our potential is constructed, and proceed to compare it with experimental PVT data from four sources; with vapor pressures and coexisting densities from two sources and with recent enthalpy data. Comparisons are also made with the predictions of the global equation of state of ethylene recently published by McCarty and Jacobsen [7]. From these comparisons it will be evident that our potential will serve as a useful and necessary supplement in a region where the global equation is deficient. In appendices, we present the equations defining our potential and a comparison of all data points mentioned above with the predictions of our surface. A table of the thermodynamic properties pressure, energy, entropy, enthalpy, specific heats $C_{p}$ and $C_{V}$ and speed of sound $C_{s}$, is presented along finely-spaced isochores with temperature as an entry. Tables of saturation properties are also given. The computer programs for generation of these tables are included. They contain an inversion routine which permits transformation from pressure to density as an independent variable.

For the convenience of the user concerned with custody transfer, we conclude the main body of the paper with a discussion of the uncertainties introduced in the density calculated from our surface due to limited precision in the measurement of pressure and temperature, and due to the presence of impurity. Contour plots indicating regions in P-T space to be avoided if a certain precision in density is desired, are included in this paper. 
Our work has been part of a joint industry-U.S. Government project to determine the thermodynamic properties of ethylene.

\section{Fundamental Equation}

At a critical point, thermodynamic behavior is anomalous. The critical point is characterized by the facts that the compressibility $K_{T}=-(1 / V)(\partial V / \partial P)_{T}$ and the specific heat $C_{p}$ diverge and the coexisting densities $\rho_{\ell}, \rho_{V}$ become equal. We write, asymptotically near the critical point,

$$
\begin{aligned}
& K \star T=\Gamma\left|\Delta T^{*}\right|^{-\gamma}, \\
& \Delta \rho^{*}=B\left|\Delta T^{*}\right|^{\beta} .
\end{aligned}
$$

Here asterisks denote reduced properties, while $\Delta T^{*} \equiv\left(T-T_{C}\right) / T_{C}$ and $\Delta \rho^{\star} \equiv\left(\rho_{\ell^{-}} \rho_{v}\right) / 2 \rho_{C}$. For classical equations of state of the type of van der Waals' equation, the critical exponent $Y$ equals $1, \beta$ equals $1 / 2$. In real fluids, $\gamma$ equals about 1.24 and $B$ is of the order 1/3. The critical-point scaling laws are constructed so that they yield the correct critical exponents; although one can define as many critical exponents as one sees fit, only two of them are independent. Their values are given by theory and they are the same for all fluids $[3,4]$.

Our fundamental equation incorporates the scaling laws. The thermodynamic potential used in our work is that introduced by Ley-Koo and Green [8]. It is the dependent variable $\tilde{P}=P \star / T^{*}$ as a function of $\tilde{\mu}=\mu^{\star} / T^{\star}$ and $\tilde{T}=-1 / T^{\star}$. Here $P$ is the pressure, $\mu$ is the chemical potential and $T$ the absolute temperature. Asterisks denote reduced properties: $P^{*}=P / P_{C}, T^{*}=T / T_{C}$ and $\mu^{*}=\mu p_{c} / P_{C}$, where $P_{C}$ is the critical pressure, $T_{c}$ the critical temperature and $\rho_{C}$ the critical density. In terms of this potential, we have

$$
\begin{aligned}
& (\partial \tilde{P} / \partial \tilde{T})_{\tilde{\mu}}=\tilde{U}, \\
& (\partial \tilde{P} / \partial \tilde{\mu})_{\tilde{T}}=\tilde{\rho},
\end{aligned}
$$

where $\tilde{U}$ is the reduced energy density $\tilde{U}=U * / V^{*}$ and $\tilde{\rho}$ the reduced mass density, while the other thermodynamic functions: Helmholtz free energy density $\tilde{A}=A^{*} / V^{*}$, enthalpy density $\tilde{H}=H * / V *$ and entropy density $\tilde{S}=S * / V *$ follow from

$$
\begin{aligned}
& \tilde{A}=\tilde{\rho}-\tilde{\mu}-\tilde{P}, \\
& \tilde{H}=\tilde{P}-\tilde{U} \tilde{T}, \\
& \tilde{S}=\tilde{H}-\tilde{\rho} \tilde{\mu} .
\end{aligned}
$$

Since all variables are intensive, the coexistent phases collapse onto a single curve, which, in the $\tilde{\mu}, \tilde{T}$ plane, can be parametrized by the analytic relation

$$
\tilde{\mu}_{0}(\tilde{T})=\tilde{\mu}_{c}+\Sigma \tilde{\mu}_{j}(\Delta \tilde{T})^{j} \text {, }
$$

where $\Delta \tilde{T}=\left(T-T_{C}\right) / T$. In scaling, the physical variables $\tilde{\mu}, \tilde{T}$ are transformed to a new 
coordinate system, one axis of which is the curve $\tilde{\mu}_{0}(\tilde{T})$, while the other intersects the first at the critical point. The two scaling variables are linear combinations of $\tilde{\mu}_{\tilde{\mu}} \tilde{\mu}_{0}$ and $\Delta \tilde{T}$ :

$$
\begin{aligned}
& u_{\mu}=\tilde{\mu}(\tilde{T})-\tilde{\mu}_{0}(\tilde{T})=\Delta \tilde{\mu} / a, \\
& u_{t}=\Delta \tilde{T}+c \Delta \tilde{\mu},
\end{aligned}
$$

where a is a constant related to the scale of $\tilde{\mu}$. The term $c \Delta \tilde{\mu}$ breaks the liquid-vapor symmetry.

The potential $\tilde{\mathrm{P}}$ is decomposed into two parts, one part, $\tilde{\mathrm{P}}_{\text {reg }}$, that is regular in the scaling variables and one, $\Delta \tilde{P}$, that contains the critical anomalies with correct power-law dependence. He have assumed for $\tilde{P}_{\text {reg }}$ :

$$
\begin{aligned}
& \tilde{P}_{\text {reg }}=\tilde{P}_{0}(\tilde{T})+\Delta \tilde{\mu}+\tilde{P}_{11} \Delta \tilde{\mu} \Delta \tilde{T}, \\
& \tilde{P}_{0}(\tilde{T})=1+\sum_{j=1} \tilde{P}_{j}(\Delta \tilde{T})^{j} .
\end{aligned}
$$

$\Delta \tilde{P}$, in scaled form, is given by

$$
\Delta \tilde{P}=a k_{0}\left|u_{t}\right|^{\beta(\delta+1)} g_{0}\left(\frac{u_{\mu}}{\left|u_{t}\right|^{\beta \delta}}\right)+a k_{1}\left|u_{t}\right|^{\beta(\delta+1)+\Delta_{1}} g_{1}\left(\frac{u_{\mu}}{\left|u_{t}\right|^{\beta \delta}}\right) .
$$

The first term represents asymptotic scaling, the second a correction to scaling. The critical exponents $\beta$ and $\delta$ are those characterizing the shape of the coexistence curve and the shape of the critical isotherm, respectively. The leading term contains one more adjustable constant $k_{0}$, while the function $g_{0}$ is universal, i.e., the same for all fluids. The correction term contains a new independent critical exponent $\Delta_{1}$, also given by theory, and a new free constant $k_{1}$. The function $g_{7}$ is the same for all fluids.

All thermodynamic functions can be given in algebraically closed form by the device of transformation to parametric variables [9]

$$
\begin{aligned}
\Delta \tilde{\mu}=a u_{\mu} & =r^{\beta \delta} a \theta\left(1-\theta^{2}\right), \\
u_{t} & =r\left(1-b^{2} \theta^{2}\right) .
\end{aligned}
$$

The variable $r$ measures "distance from the critical point", while $\theta$ is measured along a contour of constant $r$ and reaches the values +1 on the phase boundary; $b^{2}$ is a constant predicted to be universal. The critical anomalies are all imbedded in the $r$-dependence while the dependence of the thermodynamic functions on $\theta$ is always in the form of simple polynomials or their ratios. All relevant equations are given in Appendix $A$. Note that the functions are listed separately for the one- and two-phase regions.

The constants defining our potential were obtained as follows. The critical exponent values $\beta, \delta, \Delta_{1}$ are those predicted by theory [10]. The critical parameters, the four 
parameters in the scaling function: $a, k_{0}, k_{1}, c$ and the three "background" parameters $\tilde{P}_{1}$, $\tilde{P}_{2}$, and $\tilde{P}_{11}$ were determined by a fit to the PVT data of Hastings et al. [5]* while we imposed the condition that the slope of the vapor pressure curve at the critical point be that of the vapor pressure data of Levelt Sengers and Hastings [11], that the critical point 1 ie on the yapor pressure curve and that the diameter of the coexistence curve be that measured by Douslin and Harrison [12]. This implies that the values of $T_{C}$ and $P_{c}$ are constrained to be on the vapor pressure curve and cannot be fitted independently. The constant $b^{2}$, which is expected to be universal, was set equal to that found for steam [4], consistent with the theoretical value to within the latter's uncertainty. This value of $b^{2}$ (Table I) is, however, different from that used in [5]. As a consequence, all adjustable parameters except the critical constants $\rho_{C}, P_{C}$ and $T_{C}$, differ from those reported in [5]. Two background parameters $\tilde{\mu}_{2}, \tilde{\mu}_{3}$ were determined by a fit to the speed-of-sound data of Gammon [6]. The other two, $\tilde{\mu}_{c}$ and $\tilde{\mu}_{1}$, are related to the zeros for energy, entropy and enthalpy. They were fixed by equating the energy and enthalpy at $288.15 \mathrm{~K}$ and $7 \mathrm{~mol} / \mathrm{dm}^{3}$ to those derived from their PVT data [12] by Harrison and Douslin [13]. The parameters characterizing our potential are listed in Table I. The range of validity of the potential is indicated in Fig. 1.

\section{The Equation of State}

We compare the predictions of our potential for the equation of state of ethylene with experiment. The experimental data are those of Hastings et al. [5] for a $99.993 \%$ pure sample, to which the potential was fitted, those of Douslin and Harrison [12], Trappeniers et al. [14] and Thomas and Zander [15,16]. Older data have been adequately reviewed in the IUPAC formulation of Angus et al. [17], and in the reviews of Date et al. [18] and Vashchenko et al. [19].

The potential represents the data of Hastings et a1. with a reduced chi-square of 3.6 if the uncertainties are estimated as $5 \times 10^{-5} \mathrm{MPa}$ in pressure, $3 \times 10^{-3} \mathrm{~mol} / \mathrm{dm}^{3}$ in density and $5 \times 10^{-4} \mathrm{~K}$ in temperature. It is to be kept in mind that our data were fitted to about twice these close tolerances even though the deviation plots of Fig. 2 show occasionally large density deviations. The percentage differences in density, $100\left(\rho\right.$ exp. ${ }^{-\rho}$ calc. ${ }^{/ / \rho} \exp$. , between the data, $\rho_{\text {exp. }}$, and our surface, $\rho_{\text {calc. }}$, assuming the pressures to be the same, and, alternatively, the percentage differences in pressure $100\left(P_{\text {exp. }}{ }^{-P_{c a l c}}\right) / P_{\text {exp. }}$, assuming the densities to be the same, are shown for the various isotherms in Figs. 2 and 3 . At $298.15 \mathrm{~K}$ the surface represents our own data to well within $0.1 \%$ in density. The agreement between the data sets of [5], [12] and [14] is almost within the $0.1 \%$ tolerance originally set as a goal for the U.S. Government - Industry joint project on properties of ethylene.

\footnotetext{
*The bulk of the experimental PVT data were taken near, but not necessarily exactly at, ihe temperature values of $30^{\circ} \mathrm{C}, 25^{\circ} \mathrm{C}, 20^{\circ} \mathrm{C}, 16^{\circ} \mathrm{C}, 12.5^{\circ} \mathrm{C}$ and $10^{\circ} \mathrm{C}$. In Ref. [5], the temperatures were reported at these round values, and small adjustments in the experimental pressures had been made accordingly. The fit presented here, however, is to the data as measured (except for the four near-critical isochores that had been reported earlier [11]); these unadjusted data are listed in Table B1.
} 
The data set of Thomas and Zander $[15,16]$ shows more scatter than the other three sets, without, however, showing systematic departures from our formulation. The pressure deviations are similarly small at this temperature, with a tendency to grow in the highly compressed fluid, as is to be anticipated (Fig. 3). When the critical point is approached, the density departures between the data sets, and their departures from the surface, increase dramatically, while the pressure deviations remain more or less the same. At the temperatures from 0.7-3.7 $\mathrm{K}$ above the critical temperature, the density departures reach values larger than $0.5 \%$ and on isotherms spanning the critical the largest departure is $15 \%$. The pressure deviations, however, remain as well-behaved as at $298.15 \mathrm{~K}$ if not better, the departures being of the order of $0.02 \%$ in the range around critical (Fig. 3 ). This is a good illustration of the difference between "density" and "field" variables in the vicinity of a critical point. ("Density" variables are different in coexisting phases while "field" variables are the same.) Due to the strong divergences of the compressibility and expansion coefficients, small systematic differences in temperature and pressure scales between the various laboratories are amplified when the densities are compared. Furthermore, the effect of impurity is largest at the critical point, and small differences in sample composition can lead to large variations in density.

A study of the plots in Fig. 2 will convince the reader that most of the region covered in this report should be avoided if an accuracy of $0.1 \%$ in density is desired in custody transfer. Even if our surface were perfect, the finite precision of industrial pressure and temperature measurement will result in large errors if the density is calculated from the equation of state.

Appendix B. Table BI, contains the deviations of the PVT data sets [5], [12], [14], [15] and [16] from the present formulation.

\section{Critical Parameters}

The critical temperature and density of ethylene were measured by Moldover [20] by the method of visual observation of the meniscus disappearance. His results are given in Table II. The pressure given in his paper was a result of early vapor pressure measurements in the apparatus of Hastings et a1. Later, but prior to publications [11], [5], a small temperature calibration error was found in that work. The critical pressure reported by Moldover should therefore be disregarded.

The critical parameters reported by Douslin and Harrison [12] and those determined by us are also listed in Table II. These parameters are results of an analysis of PVT data. As a general rule, such analyses give set of $P_{C}$, $T_{C}$ values that are consistent with the vapor pressure curve but do not determine the value of $T_{C}$ sharply. A more accurate value of $T_{c}$ can be obtained by analyzing coexistence - curve data [12]. Coexistence curve data resulting from PVT data, however, rapidly decline in accuracy as the critical point is approached. This is true for both the data of Douslin and Harrison, and those of Hastings et al. It is therefore gratifying to see how well the critical temperatures and densities obtained by PVT data analysis agree with the direct observation by Moldover. 


\section{Coexistence Properties}

Vapor pressures from 279.15 to the critical temperature are compared in Fig. 4 . The quantity $\Delta P$ equals $P_{\text {exp. }}{ }^{-P}$ calc. Data sources are those of Hastings and Levelt Sengers [11] and those of Douslin and Harrison [12]. These two data sets were shown to agree within combined error of $10^{-4} \mathrm{MPa}$ at temperatures below $240 \mathrm{~K}$ [11]; the departures are systematic at higher temperatures, and reach a maximum of $7 \times$ $10^{-4} \mathrm{MPa}$ at the critical point. A satisfactory explanation for the difference has not been given. It is larger than expected on the basis of the agreement of pressure scales $(1$ in 20,000) and temperature scales (1 mK) and not quite of the form expected for a difference in purity [5]. Nevertheless, the agreement between the data sets [11] and [12], better than 2 parts in $10^{4}$, is an order better than that between these sets and the older data (cf. [12], p. 311). Table BII in Appendix B contains the deviations of vapor pressure data sets [11] and [12] from our formulation.

Coexisting densities of Refs. [5] and [12] are compared with our formulation in Fig. 5 and in Table BIII. The quantity $\Delta p$ equals $\rho_{\text {exp. }}{ }^{-p}$ calc. . The agreement between the two data sets and the formulation, to $\pm 0.02 \mathrm{~mol} / \mathrm{dm}^{3}$, is satisfactory in this region close to the top of the coexistence dome.

6. Speed of sound

As we mentioned before, we determined the parameters $\tilde{\mu}_{2}, \tilde{\mu}_{3}$ in our potential from a fit to Gammon's speed-of-sound data [6], accepting his density and temperature values as independent variables. It should be stressed that the anomalous contribution to the speed of sound that drives it to zero at the critical point has been completely predicted from the part of the potential that was determined by the PVT data alone. A substantial loss of accuracy is to be expected when second derivatives are calculated from the potential. We therefore consider the result of our procedure, a prediction of the speed of sound that is generally well within $1 \%$ from experiment, as very satisfactory. A global view of the speed of sound within the range of our correlation is shown in Fig. 6. Fig. 7 shows the percentage departures of the speed of sound, 100 ( $\left.C_{\text {exp. }}{ }^{-} C_{\text {calc. }}\right) / C_{\text {exp. }}$, from our surface. It should be mentioned that we had to omit a few of Gammon's data points because our surface predicted them to be inside the two-phase region. Also, we omitted the data points on the critical isotherm within $\pm 10 \%$ from the critical density, these points being heavily affected by dispersion.

The prediction of the speed of sound is about an order of magnitude less accurate than the precision of the data as estimated from Gammon's assessment of uncertainties in density, temperature and speed of sound. Permitting more than two of the model parameters to be freely adjustable did not improve the precision of the fit by more than a factor of 2 .

Table BIV in Appendix B contains a listing of the departures of the experimental data from the present formulation.

\section{Enthalpy}

Fan [21] recently obtained for ethylene values of the enthalpy and specific heat at constant pressure in a range around the critical point. The data were obtained in a carefully designed flow calorimeter with considerable precision. The calorimeter measures enthalpy changes along selected paths, isothermal or isobaric. The independent variables are pressure and temperature. A number of isobaric enthalpy differences were obtained in the range of our formulation. We have calculated with our equation the densities corresponding to Fan's temperatures and pressures, and then predicted the enthalpy differences at isobaric conditions for the observed inlet and outlet temperatures. Experiment and prediction are compared in Table BV. The agreement is not good. The differences are of the order of $0.5 \mathrm{~J} / \mathrm{g}$ or 14 $\mathrm{J} / \mathrm{mol}$, whereas our predicted enthalpies agree with those calculated by Harrison and Douslin on the level 
of a few $\mathrm{J} / \mathrm{mol}$ (cf. Section 8). Of course, a small offset in temperature or pressure scales between the calorimetric experiment and the PVT experiments will have severe consequences for the density derived from the measured pressures and temperatures; this, in turn, may affect the predicted enthalpy differences. We have varied the pressures reported by Fan by amounts of the order of $0.1 \%$, and the temperatures by a few $0.01 \mathrm{~K}$. Neither variation improved the agreement of prediction and experiment significantly. We must, therefore, reluctantly conclude that the experimental enthalpy differences are not completely consistent with the PVT data. The value of the latent heat reported by Fan, $102.8 \mathrm{~J} / \mathrm{g}$ at $4.8212 \mathrm{MPa}$, however, agrees closely with our value of $102.8 \mathrm{~J} / \mathrm{g}$ and differs from that of Harrison and Douslin, 103.3 $\mathrm{J} / \mathrm{g}$ by an amount corresponding to $25 \mathrm{mK}$ in temperature.

\section{Other Formulations}

A recent comprehensive correlation of the properties of ethylene, in the form of an equation of state based on the same data sets considered in this paper, is that of McCarty and Jacobsen [7]. It was preceded by several other correlations, such as the IUPAC formulation by Angus et al. [17], which formulation is based on a part-graphical, part-analytic representation of the PVT properties of ethylene obtained prior to 1972; and the correlation obtained by graphical means by Harrison and Dous1in [13] of their own data. The dashed curves in Figs. 2 and 3 indicate the density and pressure departures of the McCarty-Jacobsen formulation from our own; here $\Delta \rho(\%)=100\left(\rho_{M J}-\rho_{c a l c}\right) / \rho_{M J}$, and analogously for $\triangle P(\%)$. Since the Helmholtz function of McCarty and Jacobsen is analytic, it has difficulty representing the flatness of near-critical isotherms, as was recognized by the authors. At $298.15 \mathrm{~K}$ the departures, though systematic, are 1 imited to a maximum of $0.3 \%$ in density, but as the temperature approaches the critical the departures become more severe. At temperatures within $1 \mathrm{~K}$ from critical, where our formulation agrees with all data to well within $0.1 \%$ in pressure, the McCarty-Jacobsen formulation departs by several tenths of a percent in pressure in a systematic fashion. These differences are magnified when derived properties are considered. An example is shown in Fig. 8 for the enthalpy of ethylene, where the departures of the McCarty-Jacobsen enthalpy from that predicted by our surface are shown along the 283.15 $\mathrm{K}, 288.15 \mathrm{~K}$, and $298.15 \mathrm{~K}$ isotherms. Towards completion of this work, a new classical thermodynamic surface was constructed by Jacobsen and Jahangiri. For comparison with our equation, we refer to that work [22]. This new classical surface is considerably more accurate than that of [7].

The graphical method of Harrison and Douslin, if applied with care to excellent data, should be expected to give better results that can be obtained with a fit to an empirical thermodynamic surface that is seldom quite true to the data. A possible exception is the near-critical regime: the anomalous curvature of isochores may be missed by graphical techniques and by the analytic surface alike, but is correctly incorporated in our potential. The intercomparison in Fig. 8, however, shows that the enthalpies calculated by Harrison and Douslin do not depart from our surface by more than a few $\mathrm{J} / \mathrm{mol}$ anywhere, while the departures of the analytical McCarty-Jacobsen surface peak at more than $20 \mathrm{~J} / \mathrm{mol}$ at nearcritical temperatures and densities.

The departures from our surface of the derived properties according to the IUPAC formulation show strong systematic trends. This formulation was based on older data and was never claimed to possess high quality in the critical region.

First and second derivatives according to the McCarty-Jacobsen formulation are compared with our own in Figs. 9,10; here $\Delta H=H_{M J}{ }^{-H}$ calc. and similarly for $U, S$, where calc. refers to our surface. The first derivatives $U, H$ and $S$ have somewhat different density dependence in the two formulations. Moreover, appreciable departures build up around the critical density and near the critical temperature. The departures of $C_{p}, C_{v}$ and the speed of sound are substantial; here $\Delta C_{v}(\%)=100\left(C_{v}{ }^{\prime} M J-C_{v, c a l c}\right) / C_{v, c a l c}$, 
and analogously for $\Delta C_{p}, \Delta C_{s}$, where calc. refers to our surface. They are about $1 \%$ at $298.15 \mathrm{~K}, 5-10 \%$ at $288.15 \mathrm{~K}$ and $20-40 \%$ at $283.15 \mathrm{~K}(0.8 \mathrm{~K}$ above critical). An analytic representation cannot describe the divergence of $C_{V}$ and the accompanying decline to zero of the (thermodynamic) speed of sound while the divergence of $C_{p}$ is not strong enough; so these large departures are not surprising. The authors themselves did not claim accuracy in the critical region. Since we have represented the speed-of-sound data to about $1 \%$ in accuracy, we are confident that our model represents thermodynamic behavior, such as a weakly diverging $C_{V}$, accurately.

A formulation of the thermodynamic properties of ethylene in the critical region was recently published by Nehzat et al. [23]. The formulation is a nonanalytic equation of state that incorporates some features of scaled equations, such as a near-cubic coexistence curve and a critical isotherm of degree near 5 . The curvatures of the isochores, and the specific heat $C_{V}$, however, do not exhibit the predicted weak divergence in the one-phase region. The equation has a higher-order anomaly at the critical isochore; it is not integrable in closed form so that the thermodynamic functions $H, G$ and $S$ must be calculated by numerical integration. The equation of state contains 16 adjustable parameters and claims validity in a density range of 4.5 to $11 \mathrm{~mol} / \mathrm{dm}^{3}$ and 280.15 to $284.15 \mathrm{~K}$. Additional constants in the thermodynamic functions were obtained by "matching" at each isotherm with the entropy and Gibbs function of Harrison and Douslin along the isochore at $4.5 \mathrm{~mol} / \mathrm{dm}^{3}$. For comparison, our equation of state contains 10 constants (4 more for determining the thermodynamic functions), while it is valid from 5.8 to $10.5 \mathrm{~mol} / \mathrm{dm}^{3}$ and from $279-300$ $K$. The equation of state of Nehzat et al. fits the PVT data of Dous 1 in in the $4 \mathrm{~K}$ strip to close tolerance.

As far as derived properties are concerned, we compare their tabular values of $C_{V}$ in the one- and two-phase region with our surface in Figs. 11 and 12. In the two-phase region the two formulations agree as to the values of $C_{V}$ as demonstrated in Fig. 12. In the one-phase region, however, the $C_{V}$ values implied by the equation of Nehzat et al. differ appreciably from the behavior predicted from our surface as illustrated in Fig. 11. In fact, along isotherms close to the critical temperature the $C_{V}$ of Nehzat et a1. has an anomalous jump at the critical density. We do not believe that the $C_{V}$ predictions of [23] correspond with physical reality. In the absence of experimental $C_{v}$ data for ethylene, we can only argue that there is substantial evidence for many fluids that $C_{v}$ diverges; furthermore, in the case of steam, where $C_{v}$ data are available, our scaled equation represents the data at the various isochores closely [4].

The only experimental data related to $C_{v}$ for ethylene are those of Gammon for the speed of sound [6]. Nehzat et al. predict this quantity on the coexistence curve. In Fig. 13, we compare their and our predictions for the speed of sound of liquid ethylene with the data of Gammon [6]. This comparison confirms that the second derivatives are not accurately represented by the equation of Nehzat et a1. [23].

9. The Effect of Pressure and Temperature Errors on Density

In custody transfer, the density of ethylene will be calculated from its measured pressure and temperature. Since these quantities are measured with limited accuracy, an error in density will result that is given by

or

$$
\frac{\delta \rho}{\rho}=\left(P K_{T}\right) \frac{\delta P}{P},
$$

$$
\frac{\delta \rho}{\rho}=-\alpha_{P} \delta T=-K_{T}\left(\frac{\partial P}{\partial T}\right)_{V} \delta T .
$$


In view of the strong divergence of the compressibility $\mathrm{K}_{\mathrm{T}}$ and the expansion coefficient $\alpha_{\mathrm{p}}$, the density error resulting from inaccuracy in the measurement of $P$ or $T$ is a maximum at the critical point. We show the region in P-T space that should be avoided if an accuracy of $0.1 \%$ in density is desired and the pressure is measured with an accuracy of $0.1,0.05$ and $0.02 \%$, respectively, (Fig. 14); or the temperature is measured to $0.02,0.01$ or $0.005 \mathrm{~K}$, respectively, (Fig. 15).

10. The Effect of Impurity on Density

The pressure formulation of the equation of state of ethylene is based on the PVT data obtained for 99.993\% pure ethylene, with ethane as the prime impurity. The formulation permits the calculation of the density of an ethylene sample from its pressure and temperature. If impurities are present in the sample of interest, the predicted density will differ from the actual one. It is of interest to know what effect a given impurity has on density, and how the effect varies with the temperature and pressure.

Under simplifying assumptions, this question can be answered. We will assume that the impure fluid is in corresponding states with the pure host, and that the impure system displays pseudo-critical constants that are shifted from those of the pure host by amounts linear in concentration:

$$
\begin{aligned}
& T_{x c}=T_{c}(1+a x), \\
& P_{x c}=P_{c}(1+b x), \\
& V_{x c}=V_{c}(1+(a-b) x) .
\end{aligned}
$$

Both assumptions are reasonable and readily justifiable in dilute mixtures not close to the critical line; the only difficulty with the model is the actual assignment of the values of a and b, parameters characteristic of the host-impurity interaction. They can be determined from the critical parameters of the pure components in the case that the critical points are connected by a continuous critical 1ine. For the sake of developing our estimate of the density error introduced by impurity, we will consider these parameters a,b as known.

An equation for the impurity-induced density error was derived by one of us [5]. It was obtained by applying the law of corresponding states to the pure host and the dilute mixture at the same pressure and temperature. The following result was obtained for the change in density:

$$
\begin{aligned}
\frac{\delta \rho}{\rho} & =x\left[(a-b)+b P K_{T}-a T \alpha_{P}\right] \\
& =x\left[(a-b)+b P K_{T}-a\left(P K_{T}\right)\left(\frac{T}{P} \frac{\partial P}{\partial T}\right)\right],
\end{aligned}
$$

where $K_{T}$ is the compressibility, $\alpha_{p}$ the expansion coefficient of the pure host at $T, P$. It is clear that Eq. (11) permits calculation of the impurity-induced error in density at any point in P-T space once the constants $a, b$ are known. The relation (11) should not be applied too close to the critical point since the coefficient of the term in $x$ diverges. More seriously, an expansion in $x$ has as little justification as one in $\rho$, which is known to be invalid at the critical point.

A scrutiny of Eq. (11) immediately reveals that impurity-induced density errors will be 1 argest near the critical point. This is a consequence of the fact that both $K_{T}$ and $a_{p}$ diverge strongly at this point. The precise nature of this density error will, of course, depend on the values of a and $b$. Since these values form the least-known part of our model, we will consider only a few extreme cases.

(1) $a=-0.5, b=0$ : a highly volatile impurity that depresses the critical the critical temperature of the mixture strongly without affecting the critical pressure. 
(2) $a=+0.5, b=0$ : a highly nonvolatile impurity that increases the critical temperature of the mixture strongly without affecting the critical pressure.

The cases (1) and (2) have opposite effects on the density. The impurity-induced density error is given by

$$
\frac{\delta \rho}{\rho}= \pm 0.5 \times\left[1-\left(P K_{T}\right)\left(\frac{T}{P} \frac{\partial P}{\partial T}\right)\right]
$$

where the + sign refers to case 1 , the sign to case 2 . In Fig. 16 , we show the region in P-T space that should be avoided in custody transfer if an accuracy in density of $0.1 \%$ is desired and if various amounts of an impurity very different in volatility from ethylene are present.

11. Tables of Thermodynamic Properties

In Appendix $C$ we present the thermodynamic properties of ethylene in the critical region. It was not possible to organize this table along isobars, as in [6]. The extremely rapid variation of the "density"-like properties $\rho, U, H, S$ with $P$ and T would preclude interpolation in such a table. We have therefore tabulated the properties along isochores, or at constant density, using temperature as the second entry (Table CI). In addition, we present tables of saturation properties first with temperature, then with pressure as an entry (Tables CII, CIII).

The computer program required for the generation of thermodynamic properties of ethylene is listed in Appendix D. Appendix $E$ contains units and conversion factors.

\section{Acknowledgements}

R.D. MicCarty, B.E. Gammon and Yu-Chun Fan provided us with results of their work prior to publication. Constructive observations by R.T. Jacobsen and M. Jahangiri at various stages of this work led to a number of improvements. We have benefitted from the assistance of J.S. Gallagher in an earlier stage of this project. Computer time for this project was provided by the Computer Science Center of the University of Maryland.

\section{References}

[1] Sengers, J.V. and Levelt Sengers, J.M.H., "The critical region", Chem. and Eng. News 46, $104,1968$.

[2] Levelt Sengers, J.M.H., "Scaling predictions for thermodynamic anomalies near the gas-liquid critical point", Ind. Eng. Fund. $\underline{9}, 470,1970$.

[3] Levelt Sengers, J.M.H., Morrison, G. and Chang, R.F., "Critical behavior in fluids and fluid mixtures", Fluid Phase Equilibria 14, 19, 1983.

[4] Levelt Sengers, J.M.H., Kamgar-Parsi, B. and Sengers, J.V., "Thermodynamic properties of steam in the critical region", J. Phys. Chem. Ref. Data, 12, 1, 1983.

[5] Hastings, J.R., Levelt Sengers, J.M.H. and Balfour, F.W., "The critical-region equation of state of ethylene and the effect of small impurities", J. Chem. Thermodynamics 12, 1009, 1980.

[6] Gammon, B.E., "Velocity of sound in ethylene", presented at the Advisory Committee Meeting of the Joint Industry-Government Ethylene Project, Boulder, Colorado (Dec. 6, 1978). 
[7] McCarty, R.D. and Jacobsen, R.T., "An equation of state for fluid ethylene", NBS Tech. Note 1045, (Nat'1 Bur. Stand., Washington, DC, 1981).

[8] Ley-Koo, M. and Green, M.S., "Consequences of the renormalization group for the thermodynamics of fluids near the critical point", Phys. Rev. A 23, 2650, 1981.

[9] Schofield, P., "Parametric representation of the equation of state near a critical point", Phys. Rev. Letters 22, 606, 1969.

[10] Le Guillou, J.C. and Zinn-Justin, J., "Critical exponents from field theory", Phys. Rev. B 21, 3976, 1980.

[11] Hastings, J.R., and Levelt Sengers, J.M.H., "Vapor pressure, critical pressure, and critical isochore of ethylene", in Proceedings 7 th Symposium on Thermophysical Properties, Cezairliyan, A., ed., (American Society of Mechanical Engineers, New York, 1977) p. 794.

[12] Douslin, D.R., and Harrison, R.H., "Pressure, volume, temperature relations of ethylene", J. Chem. Thermodynamics $\underline{8}, 301$ (1976).

[13] Harrison, R.H. and Douslin, D.R., "Derived thermodynamic properties of ethylene", J. Chem. Eng. Data 22, 241977.

[14] Trappeniers, N.J., Wassenaar, J., and Wolkers, G.J., "Isotherms and thermodynamic properties of ethylene at temperatures between 0 and $150 \mathrm{C}$ and at densities up to 500 amagat", Physica $82 \mathrm{~A}, 305$ (1976).

[15] Thomas, W., Zander, M., Quietzsch, G., and Hartmann, H., "Dichte des Aethylens fuer Temperaturen von $-30 \mathrm{C}$ bis $80 \mathrm{C}$ bei Drucken bis zu 100 bar," Herausgeber Hoechst AG, Frankfurt/M-Hoechst (1976).

[16] Thomas, W., and Zander, M., "Pressure-density-temperature measurements of ethylene", Int. J. Thermophysics 1, 383 (1980).

[17] Angus, S., Armstrong, B., de Reuck, K.M., Featherstone, W., and Gibson, M.R., International Thermodynamic Tables of the Fluid State, Ethylene, 1972, International Union of Pure and Applied Chemistry, Division of Physical Chemistry, Commission on Thermophysic and Thermochemistry, Thermodynamic Tables Project, Butterworths, London (1974).

[18] Date, K., Watanabe, J., and Uematsu, M., "Evaluation of P-V-T properties data, the most probable values of the compressibility factor of gaseous ethane and ethene", Review of Phys. Chem. Japan $\underline{43}$, 92 (1973).

[19] Vashchenko, D.M., Voinov Yu, F., Voityuk, B.V., Dregulyas, E.K., Kolomiets, Ya., A., Labinov, S.D., Morozov, A.A., Neduzhij, I.A., (Principal Author), Provotar, V.P., A. Yu. Soldatenko, Storozhenko, E.I., and Khmara, Yu. I., Thermodynamic and transport properties of ethylene and propylene (translated from the Russian as published by the state Committee of Standards of the Soviet Ministry, U.S.S.R., State Officer of Standards and Reference Data, Series: Monograph No. 8, 1979), Office of Standard Reference Data, National Bureau of Standards U.S. Department of Commerce, Washington, D.C.

[20] Moldover, M.R., "Visual observation of the criical temperature and density: $\mathrm{CO}_{2}$ and $\mathrm{C}_{2} \mathrm{H}_{4}$ ", $\mathrm{J}$. Chem. Phys. 미, 1766 (1979).

[21] Fan, Yu-Chun, "Accurate Calorimetric Determination in the Critical Region of Ethylene", Ph.D. Thesis, Dept. of Chem. Engineering, University of Michigan, Ann Arbor, Michigan, 1982.

[22] Jacobsen, R.T. and Jahangiri, M., to be published.

[23] Nehzat, M.S., Ha11, K.R. and Eubank, P.T., "Thermophysical Properties of Ethylene in the Critical Region", J. Chem. Eng. Data 28, 205 (1983).

[24] Waxman, M. and Davis, H.A., Adv. in Chem. Series 182, K.C. Chao and R.L. Robinson, Jr., editors, ACS Washington, p. 285 (1979). 
Table I. Parameters in the Thermodynamic Potential for Ethylene

\section{Critical Exponents}

$$
\begin{aligned}
\beta & =0.325 \\
\delta & =4.82 \\
\Delta_{1} & =0.50
\end{aligned}
$$

\section{Critical Parameters}

Pameters in

Scaling Function

Pressure background parameters

\section{Caloric background} parameters

$$
\begin{aligned}
& T_{c}=282.3452 \mathrm{~K} \\
& \rho_{c}=7.634 \mathrm{~mol} / \mathrm{dm}^{3} \\
& P_{c}=5.0403 \mathrm{MPa} \text { (derived) }
\end{aligned}
$$

$$
\begin{aligned}
& a_{0}=19.3214 \\
& k_{0}=1.12289 \\
& k_{1}=0.54617 \\
& c=-0.007811 \\
& b^{2}=1.3757
\end{aligned}
$$

$$
\begin{aligned}
& \tilde{P}_{1}=5.3350 \\
& \tilde{P}_{2}=-18.1475 \\
& \tilde{P}_{11}=-0.166687 \text { (derived) }
\end{aligned}
$$

Table II. Critical Parameters of Ethylene

Author

Data Source

$T_{C}, K$

$P_{C}, M P a$

$\rho_{\mathrm{c}}, \mathrm{mol} / \mathrm{dm}^{3}$

Moldover [20]

visual observation of $282.344 \pm 0.004$

$\tilde{\mu}_{0}=-36.4889$

$\tilde{\mu}_{1}=-27.3955$

$\tilde{\mu}_{2}=-12.2091$

$\tilde{\mu}_{3}=-11.8802$

meniscus disappearance

Doust in [12]

PVT [12]

282.35

5.0420

$7.650+0.021$

This work

PVT [5]

282.3452

5.0403

$7.635+0.006$

7.634 
APPENDIX A: Revised and Extended Scaling Equations for the Thermodynamic

Properties of Fluids.

\section{A.1 Reduced thermodynamic quantities}

$$
\begin{aligned}
& \tilde{T}=-\frac{T c}{T}, \quad \tilde{\mu}=\frac{\mu}{T} \cdot \frac{\rho_{c} T_{c}}{P_{c}}, \quad \tilde{P}=\frac{P}{T} \cdot \frac{T_{c}}{P_{c}}, \\
& \tilde{\rho}=\frac{\rho}{\rho_{c}}, \quad \tilde{U}=\frac{U}{V} \cdot \frac{1}{P_{c}}, \quad \tilde{S}=\frac{S}{V} \cdot \frac{T_{c}}{P_{c}}, \\
& \tilde{A}=\frac{A}{V T} \cdot \frac{T_{c}}{P_{c}}, \quad \tilde{H}=\frac{H}{V T} \cdot \frac{T c}{P_{c}}, \quad \tilde{X}_{T}=\left(\frac{\partial \tilde{\rho}}{\partial \tilde{\mu}}\right)_{T}, \\
& \tilde{C}_{V}=\frac{C_{v}}{V} \frac{T_{c}}{P_{c}}, \quad \tilde{C}_{P}=\frac{C}{V} \cdot \frac{T c}{P_{c}}
\end{aligned}
$$

(T is temperature, $\mu$ is chemical potential, $P$ is pressure, $\rho$ is density, $\mathrm{U}$ is energy, $\mathrm{S}$ is entropy, $\mathrm{A}$ is Helmholtz free energy, $\mathrm{H}$ is enthalpy, $V$ is volume, $C_{V}$ is heat capacity at constant $V, C_{p}$ is heat capacity at constant P).

\section{A.2 Thermodynamic relations}

$$
\begin{aligned}
& d \tilde{P}=\tilde{U} d \tilde{T}+\tilde{\rho} d \tilde{\mu} \\
& d \tilde{A}=-\tilde{U} d \tilde{T}+\tilde{\mu} d \tilde{\rho} \\
& d \tilde{H}=-\tilde{T} d \tilde{U}+\tilde{\rho} d \tilde{\mu} \\
& d \tilde{S}=-\tilde{T} d \tilde{U}-\tilde{\mu} d \tilde{\rho}
\end{aligned}
$$


with

$$
\begin{aligned}
& \tilde{A}=\tilde{\rho \mu}-\tilde{P} \\
& \tilde{H}=\tilde{P}-\tilde{T} \tilde{U} \\
& \tilde{S}=\tilde{H}-\tilde{\rho \mu}=-\tilde{T U}-\tilde{A}
\end{aligned}
$$

\section{A.3 Fundamental equations}

$$
\begin{aligned}
& \Delta \tilde{\mathrm{T}}=\tilde{\mathrm{T}}+1 \\
& \Delta \tilde{\mu}=\tilde{\mu}-\tilde{\mu}_{0}(\tilde{\mathrm{T}}) \\
& \tilde{\mathrm{P}}=\tilde{\mathrm{P}}_{0}(\tilde{\mathrm{T}})+\Delta \tilde{\mu}+\tilde{\mathrm{P}}_{11} \Delta \tilde{\mu} \Delta \tilde{\mathrm{T}}+\Delta \tilde{\mathrm{P}}
\end{aligned}
$$

with

$$
\begin{aligned}
& \tilde{\mu}_{0}(\tilde{T})=\tilde{\mu}_{c}+\sum_{j=1}^{3} \tilde{\mu}_{j}(\Delta \tilde{T})^{j} \\
& \tilde{P}_{0}(\tilde{T})=1+\sum_{j=1}^{2} \tilde{P}_{j}(\Delta \tilde{T})^{j}
\end{aligned}
$$

A.4 Derived thermodynamic quantities

$$
\begin{aligned}
& \tilde{\rho}=1+\tilde{\mathrm{P}}_{11} \Delta \tilde{\mathrm{T}}+\left(\frac{\partial \Delta \tilde{\mathrm{P}}}{\partial \Delta \tilde{\mu}}\right)_{\Delta \tilde{T}} \\
& \tilde{U}=\frac{{ }_{\mathrm{P}}}{\mathrm{dT}}-\frac{\tilde{d}_{0}}{\mathrm{~d} \tilde{\mathrm{T}}}+\tilde{\mathrm{P}}_{11} \Delta \tilde{\mu}+\left(\frac{\partial \Delta \tilde{\mathrm{P}}}{\partial \Delta \mathrm{T}}\right)_{\Delta \tilde{\mu}} \\
& \tilde{\mathrm{X}}_{\mathrm{T}}=\left(\frac{\partial^{2} \Delta \tilde{\mathrm{P}}}{\partial \Delta \tilde{\mu}^{2}}\right)_{\Delta \tilde{T}}
\end{aligned}
$$




$$
\begin{aligned}
& \left(\frac{\partial \tilde{P}}{\partial \tilde{T}}\right)_{\tilde{P}}=\frac{d \tilde{P}}{d \tilde{T}}+\tilde{P}_{11}\left[\Delta \tilde{\mu}-\frac{\tilde{\rho}}{\tilde{X}_{T}}\right]+\left(\frac{\partial \Delta \tilde{P}}{\partial \Delta \tilde{T}}\right)_{\Delta \tilde{\mu}}-\frac{\tilde{\rho}}{\tilde{X}_{T}} \frac{\partial^{2} \Delta \tilde{P}}{\partial \Delta \tilde{\mu} \partial \Delta \tilde{T}} \\
& \frac{\tilde{c}_{v}}{\tilde{T}^{2}}=\frac{d^{2} \tilde{p}_{o}}{d \tilde{T}^{2}}-\tilde{\rho} \frac{d^{2} \tilde{\mu}_{o}}{d \tilde{T}^{2}}-\frac{\tilde{p}_{11}^{2}}{\tilde{X}_{T}}+ \\
& +\left(\frac{\partial^{2} \Delta \tilde{P}}{\partial \Delta \tilde{T}^{2}}\right)_{\Delta \tilde{\mu}}-\frac{2 \tilde{P}}{\tilde{x}_{T}} \frac{\partial^{2} \Delta \tilde{P}}{\partial \Delta \tilde{\mu} \partial \Delta \tilde{T}}-\frac{1}{\tilde{x}_{T}}\left(\frac{\partial^{2} \Delta \tilde{P}}{\partial \Delta \tilde{\mu} \partial \Delta \tilde{T}}\right)^{2} \\
& \tilde{c}_{p}=\tilde{C}_{v}+\frac{\tilde{X}_{T}}{\tilde{\rho}^{2}}\left[\tilde{p}-\tilde{T}\left(\frac{\partial \tilde{P}}{\partial \tilde{T}}\right)_{\tilde{\rho}}\right]^{2}
\end{aligned}
$$

A.5 Critical exponents

$$
\begin{array}{ll}
\alpha_{0}=\alpha, & \alpha_{1}=\alpha-\Delta_{1} \\
\beta_{0}=\beta, & \beta_{1}=\beta+\Delta_{1} \\
\gamma_{0}=\gamma, & \gamma_{1}=\gamma-\Delta_{1}
\end{array}
$$

with

$$
2-\alpha=\beta(\delta+1), \quad \gamma=\beta(\delta-1)
$$

\section{A.6 Parametric equations for singular terms}

$$
\begin{aligned}
& \Delta \tilde{\mu}=r^{B \delta} a\left(1-\theta^{2}\right) \\
& \Delta \tilde{T}=r\left(1-b^{2} \theta^{2}\right)-c \Delta \tilde{\mu}
\end{aligned}
$$




$$
\begin{aligned}
& \Delta \tilde{\mathrm{P}}=\sum_{i=0}^{1} \mathbf{r}^{2-\alpha_{i}} \mathrm{k}_{i} p_{i}(\theta) \\
& \left(\frac{\partial \Delta \tilde{p}}{\partial \Delta \tilde{\mu}}\right)_{\Delta \tilde{T}}=\sum_{i=0}^{1}\left[r^{\beta_{i k}} k_{i} \theta+c I^{1-\alpha_{i a k_{i}} s_{i}(\theta)}\right] \\
& \left(\frac{\partial \Delta \tilde{p}}{\partial \Delta \tilde{T}}\right)_{\Delta \tilde{\mu}}=\sum_{i=0}^{1} x^{1-\alpha_{i_{a k}} s_{i}(\theta)} \\
& \left(\frac{\partial^{2} \Delta \tilde{p}}{\partial \Delta \tilde{\mu}^{2}}\right)_{\Delta \tilde{T}}=\sum_{i=0}^{1}\left[r^{-\gamma_{i} k_{i}} u_{i}(\theta)+2 c r^{\beta_{i}-1} k_{i} v_{i}(\theta)+c^{2}{ }^{2}-\alpha_{i_{a k}} w_{i} w_{i}(\theta)\right] \\
& \frac{\partial^{2} \Delta \tilde{p}}{\partial \Delta \tilde{\mu} \Delta \tilde{T}}=\sum_{i=0}^{1}\left[r^{\beta_{i}-1} k_{i} v_{i}(\theta)+c r^{-\alpha} \alpha_{a k_{i}} w_{i}(\theta)\right] \\
& \left(\frac{\partial^{2} \Delta \tilde{p}}{\partial \Delta \tilde{T}^{2}}\right)_{\Delta \tilde{\mu}}=\sum_{i=0}^{1} r^{-\alpha_{i a k_{i}} w_{i}(\theta)}
\end{aligned}
$$

A.7 Auxiliary functions

$$
\begin{aligned}
& p_{i}(\theta)=p_{0 i}+p_{2 i} \theta^{2}+p_{4 i} \theta^{4} \\
& s_{i}(\theta)=s_{0 i}+s_{2 i} \theta^{2}, \quad s_{i}^{\prime}(\theta)=2 s_{2 i} \theta \\
& q(\theta)=1+\left\{b^{2}(2 \beta \delta-1)-3\right\} \theta^{2}-b^{2}(2 B \delta-3) \theta^{4} \\
& u_{i}(\theta)=\left[1-b^{2}\left(1-2 \beta_{i}\right) \theta^{2}\right] / q(\theta) \\
& v_{i}(\theta)=\left[\beta_{i}\left(1-3 \theta^{2}\right) \theta-\beta \delta\left(1-\theta^{2}\right) \theta\right] / q(\theta)
\end{aligned}
$$

17 


$$
w_{i}(\theta)=\left[\left(1-\alpha_{i}\right)\left(1-3 \theta^{-}\right) s_{i}(\theta)-B \delta\left(1-\theta^{-}\right) \theta s_{i}(\theta)\right] / q(\theta)
$$

with

$$
\begin{aligned}
& p_{0 i}=+\frac{\beta \delta-3 \beta_{i}-b^{2} \alpha_{i} \gamma_{i}}{2 b^{4}\left(2-\alpha_{i}\right)\left(1-\alpha_{i}\right) \alpha_{i}} \\
& p_{2 i}=-\frac{\beta \delta-3 \beta_{i}-b^{2} \alpha_{i}(2 \beta \delta-1)}{2 b^{2}\left(1-\alpha_{i}\right) \alpha_{i}} \\
& p_{4 i}=+\frac{2 \beta \delta-3}{2 \alpha_{i}} \\
& s_{0 i}=\left(2-\alpha_{i}\right) p_{o i} \\
& s_{2 i}=-\frac{\beta \delta-3 \beta_{i}}{2 b^{2} \alpha_{i}}
\end{aligned}
$$

\section{A.8 Two-phase properties}

Variables:

$$
\begin{aligned}
\theta & = \pm 1 \\
\Delta \tilde{\mu} & =0 \\
\Delta \tilde{T} & =r\left(1-b^{2}\right)
\end{aligned}
$$

Vapor pressure:

$$
\tilde{P}_{\text {vap }}=\tilde{P}_{0}(T)+\sum_{i=0}^{1} r^{2-\alpha_{i}} \quad a k_{i} p_{i}(1)
$$


Coexisting Densities:

$$
\begin{aligned}
& \frac{\tilde{\rho}_{L+} \tilde{p}_{v}}{2}=1+\tilde{p}_{11} \Delta \tilde{T}+\sum_{i=0}^{1} c r^{1-a_{i} k_{i} s_{i}(1)} \\
& \frac{\tilde{\rho}_{L}-\tilde{p}_{v}}{2}=\sum_{i=0}^{1} r^{\beta_{i}} k_{i}
\end{aligned}
$$

Helmholtz free energy:

$$
\tilde{A}=\tilde{\rho} \tilde{u}_{0}(\tilde{T})-\tilde{P}_{0}(T)-\sum_{i=0}^{1} r^{2-\alpha_{i} \cdot a k_{i} p_{i}(1)}
$$

Energy :

$$
\tilde{v}=\frac{d \tilde{P}_{0}}{d \tilde{T}}-p \frac{d \tilde{\mu}_{0}(T)}{d \tilde{T}}+\frac{1}{1-b^{2}} \sum_{i=0}^{1}\left(2-\alpha_{i}\right) r^{1-\alpha_{i}}{a k_{i}}_{i} P_{i}(1)
$$

Entropy:

$$
\begin{aligned}
\tilde{s} & =-\tilde{\rho} \tilde{\mu}_{0}(\tilde{T})+\tilde{P}_{0}(\tilde{T})-\tilde{T}\left[\frac{d \tilde{P}_{0}}{d \tilde{T}}-\tilde{\rho} \frac{d \tilde{\mu}_{0}}{d \tilde{T}}\right] \\
& +\sum_{i=0}^{1} r^{2-\alpha_{i}} a k_{i} p_{i}(1)-\frac{\tilde{T}}{1-b^{2}} \sum_{i=0}^{1}\left(2-\alpha_{i}\right) r^{1-\alpha_{i}}{a k_{i} p_{i}(1) \quad \text { (A.36) }}
\end{aligned}
$$

Specific Heat $\mathrm{C}_{\mathrm{y}}$ :

$$
\frac{\tilde{c}}{\tilde{T}^{2}}=\frac{d^{2} \tilde{p}_{o}}{d \tilde{T}^{2}}-\tilde{\rho} \frac{d^{2} \tilde{u}_{o}}{d \tilde{T}^{2}}+\left(\frac{1}{1-b^{2}}\right)^{2} \sum_{i=0}^{1}\left(2-\alpha_{i}\right)\left(1-\alpha_{i}\right) r^{-\alpha_{1}} a k_{i} p_{i}(1)
$$


In Table B I, the experimental PVT data are compared with the formulation (a) as pressure differences under the assumption that the temperatures and densities are exact and (b) as density differences under the assumption that the temperatures and pressures are exact. In Table B II, saturation pressures from two sources are compared with the formulation and in Table B III the saturation densities. In Table B IV, the speed-of-sound data [6] are compared with the theoretical predictions, and in Table $B V$ the enthalpy data. The legend for the various data sources is as follows

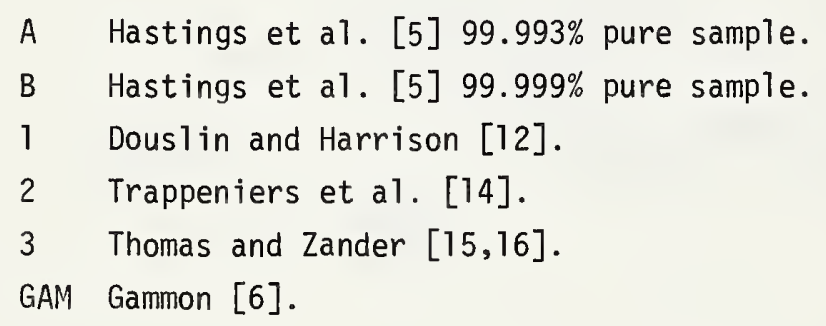


TABLE-BI. Comparison of the experimental PVT data with the formulation.

\begin{tabular}{|c|c|c|c|c|c|c|c|c|c|}
\hline \multirow[t]{3}{*}{ EXP.ID } & \multicolumn{2}{|c|}{ RATURE } & \multicolumn{3}{|c|}{ PFESSURE } & \multicolumn{4}{|c|}{ DENSITY } \\
\hline & \multirow[t]{2}{*}{$\kappa$} & \multicolumn{4}{|c|}{ MPA } & \multicolumn{4}{|c|}{ MOL/LIM3 } \\
\hline & & EXP & CALC & IIIFF & \%DIFF & EXP & CALC & IIFF & \%IIFF \\
\hline A & 298.1386 & 8.32147 & 8.32105 & .00042 & .005 & 10.542 & 10.543 & -.000 & -.00 \\
\hline A & 298.1386 & 8.32149 & 8.32105 & .00044 & .005 & 10.542 & 10.543 & -.000 & -.00 \\
\hline A & 293.1388 & 7.32940 & 7.32704 & .00236 & .032 & 10.545 & 10.548 & -.003 & -.03 \\
\hline A & 289.1390 & 6.54643 & 6.54307 & .00336 & .051 & 10.547 & 10.552 & -.006 & -.05 \\
\hline A & 283.6392 & 5.49244 & 5.48964 & .00280 & .051 & 10.550 & 10.557 & -.008 & -.07 \\
\hline A & 283.6392 & 5.49234 & 5.48964 & .00270 & .049 & 10.550 & 10.557 & -.007 & -.07 \\
\hline A & 282.3333 & 5.24755 & 5.24556 & .00199 & .038 & 10.550 & 10.557 & -.006 & -.06 \\
\hline A & 281.1393 & 5.02650 & 5.02529 & .00121 & .024 & 10.551 & 10.555 & -.005 & -.04 \\
\hline A & 280.6394 & 4.93481 & 4.93404 & .00077 & .016 & 10.551 & 10.554 & -.003 & -.03 \\
\hline A & 280.3394 & 4.88010 & 4.87961 & .00049 & .010 & 10.551 & 10.553 & -.002 & -.02 \\
\hline A & 280.0394 & 4.82562 & 4.82543 & .00019 & .004 & 10.551 & 10.552 & -.001 & -.01 \\
\hline A & 279.7394 & 4.77146 & 4.77154 & -.00008 & -.002 & 10.551 & 10.551 & .000 & .00 \\
\hline A & 298.1386 & 7.89536 & 7.90041 & -.00505 & -.064 & 10.024 & 10.017 & .007 & .07 \\
\hline A & 298.1386 & 7.89545 & 7.90041 & -.00496 & -.063 & 10.024 & 10.017 & .007 & .07 \\
\hline A & 285.6391 & 5.68452 & 5.68402 & .00050 & .009 & 10.030 & 10.032 & -.002 & -.02 \\
\hline A & 283.6392 & 5.34404 & 5.34389 & .00015 & .003 & 10.031 & 10.032 & -.001 & -.01 \\
\hline A & 282.3333 & 5.12547 & 5.12580 & -.00033 & -.006 & 10.031 & 10.029 & .002 & .02 \\
\hline A & 281.1393 & 4.92924 & 4.93023 & -.00099 & -.020 & 10.032 & 10.024 & .009 & .08 \\
\hline A & 280.8393 & 4.88062 & 4.88185 & -.00123 & -.025 & 10.032 & 10.020 & .012 & .12 \\
\hline A & 280.7393 & 4.86455 & 4.86581 & -.00126 & -.026 & 10.032 & 10.020 & .013 & .13 \\
\hline A & 298.1386 & 7.46041 & 7.46406 & -.00365 & -.049 & 9.285 & 9.278 & .007 & .08 \\
\hline A & 298.1386 & 7.46049 & 7.46406 & -.00357 & -.048 & 9.285 & 9.278 & .007 & .08 \\
\hline A & 293.1388 & 6.67996 & 6.68064 & -.00068 & -.010 & 9.288 & 9.286 & .002 & .02 \\
\hline A & 289.1390 & 6.06498 & 6.06419 & .00079 & .013 & 9.289 & 9.293 & -.003 & -.04 \\
\hline A & 285.6391 & 5.53737 & 5.53628 & .00109 & .020 & 9.291 & 9.299 & -.008 & -.09 \\
\hline A & 283.6392 & 5.24276 & 5.24217 & .00059 & .011 & 9.292 & 9.299 & -.007 & -.08 \\
\hline A & 282.3333 & 5.05492 & 5.05501 & -.00009 & -.002 & 9.292 & 9.290 & .002 & .02 \\
\hline A & 281.8393 & 4.98547 & 4.98586 & -.00039 & -.008 & 9.293 & 9.279 & .013 & .14 \\
\hline A & 281.7893 & 4.97849 & 4.97894 & -.00045 & -.009 & 9.293 & 9.277 & .016 & .17 \\
\hline A & 298.1386 & 7.29300 & 7.29536 & -.00236 & -.032 & 8.911 & 8.905 & .006 & .06 \\
\hline A & 298.1386 & 7.29308 & 7.29536 & -.00228 & -.031 & 8.911 & 8.905 & .006 & .06 \\
\hline A & 293.1388 & 6.56382 & 6.56372 & .00010 & .001 & 8.913 & 8.913 & -.000 & -.00 \\
\hline A & 289.1390 & 5.98874 & 5.98747 & .00127 & .021 & 8.914 & 8.921 & -.007 & -.08 \\
\hline A & 285.6391 & 5.49490 & 5.49353 & .00137 & .025 & 8.916 & 8.930 & -.014 & -.16 \\
\hline A & 283.6392 & 5.21907 & 5.21834 & .00073 & .014 & 8.917 & 8.932 & -.015 & -.17 \\
\hline A & 282.3333 & 5.04370 & 5.04370 & -.00000 & -.000 & 8.917 & 8.917 & .000 & .00 \\
\hline A & 282.1893 & 5.02483 & 5.02489 & -.00006 & -.001 & 8.917 & 8.913 & .004 & .05 \\
\hline A & 282.0893 & 5.01180 & 5.01191 & -.00011 & -.002 & 8.917 & 8.907 & .010 & .11 \\
\hline A & 282.0993 & 5.01308 & 5.01321 & -.00013 & -.003 & 8.917 & 8.906 & .011 & .12 \\
\hline A & 282.0893 & 5.01180 & 5.01191 & -.00011 & -.002 & 8.917 & 8.907 & .010 & .11 \\
\hline A & 282.0943 & 5.01245 & 5.01256 & -.00011 & -.002 & 8.917 & 8.908 & .010 & .11 \\
\hline A & 282.0893 & 5.01182 & 5.01191 & -.00009 & -.002 & 8.917 & 8.909 & .008 & .09 \\
\hline A & 282.0949 & 5.01251 & 5.01264 & -.00013 & -.003 & 8.917 & 8.906 & .011 & .13 \\
\hline A & 282.0946 & 5.01245 & 5.01260 & -.00015 & -.003 & 8.917 & 8.904 & .013 & .15 \\
\hline A & 282.0942 & 5.01246 & 5.01255 & -.00009 & -.002 & 8.917 & 8.910 & .008 & .08 \\
\hline A & 282.0936 & 5.01233 & 5.01247 & -.00014 & -.003 & 8.917 & 8.905 & .012 & .14 \\
\hline A & 282.0891 & 5.01176 & 5.01189 & -.00013 & -.003 & 8.917 & 8.906 & .011 & .13 \\
\hline A & 282.0877 & 5.01156 & 5.01171 & -.00015 & -.003 & 8.917 & 8.904 & .013 & .15 \\
\hline A & 298.1386 & 7.23223 & 7.23416 & -.00193 & -.027 & 8.758 & 8.753 & .005 & .06 \\
\hline A & 298.1386 & 7.23231 & 7.23416 & -.00185 & -.026 & 8.758 & 8.753 & .005 & .05 \\
\hline A & 283.6392 & 5.21245 & 5.21172 & .00073 & .014 & 8.764 & 8.782 & -.018 & -.21 \\
\hline A & 282.2193 & 5.02695 & 5.02703 & -.00008 & -.002 & 8.764 & 8.755 & .009 & .10 \\
\hline A & $282 \cdot 3333$ & 5.04151 & 5.04152 & -.00001 & -.000 & 8.764 & 8.764 & .000 & .01 \\
\hline A & 282.1893 & 5.02316 & 5.02323 & -.00007 & -.001 & 8.764 & 8.755 & .009 & .11 \\
\hline A & 282.1893 & 5.02316 & 5.02323 & -.00007 & -.001 & 8.764 & 8.755 & .009 & .11 \\
\hline A & 282.3960 & 5.04961 & 5.04952 & .00009 & .002 & 8.764 & 8.771 & -.007 & -.08 \\
\hline A & 282.1708 & 5.02097 & 5.02090 & .00007 & .001 & 8.764 & 8.774 & -.010 & -.11 \\
\hline A & 282.1915 & 5.02346 & 5.02351 & -.00005 & -.001 & 8.764 & 8.758 & .006 & .07 \\
\hline A & 282.1909 & 5.02335 & 5.02343 & -.00008 & -.002 & 8.764 & 8.753 & .011 & .12 \\
\hline
\end{tabular}




\begin{tabular}{|c|c|c|c|c|c|c|c|c|c|}
\hline \multirow[t]{3}{*}{$E</ F \cdot I I I$} & TEMFEFIATUFIE & \multicolumn{4}{|c|}{ F'RESSURE } & \multicolumn{4}{|c|}{ IIENSITY } \\
\hline & $\kappa$ & \multirow[b]{2}{*}{$E \times F$} & \multicolumn{2}{|c|}{$M F A$} & \multirow[b]{2}{*}{ \%IFF } & \multicolumn{4}{|c|}{ MOL/DM3 } \\
\hline & & & CALC & IIIFF & & $E \times F$ & CALC & IIIFF & \%IIIFF \\
\hline A & 282.1911 & 5.02344 & 5.02346 & -.00002 & -.000 & 8.764 & 8.762 & .002 & .03 \\
\hline A & 232.1916 & 5.02346 & 5.02352 & -.00006 & -.001 & 8.764 & 8.755 & .008 & .09 \\
\hline A & 303.1500 & 7.47291 & $7.4740 \mathrm{~s}$ & -.00125 & -.017 & 7.672 & 7.669 & .003 & .04 \\
\hline A & 299.1500 & 6.88253 & 6.88293 & -.00037 & -.005 & 7.674 & 7.673 & .001 & .02 \\
\hline A & 298.1500 & 6.88257 & 6.88293 & -.000 .36 & -.005 & 7.674 & 7.673 & .001 & .02 \\
\hline A & 293.1500 & 6.29461 & 6.29429 & .00032 & .005 & 7.676 & 7.678 & -.002 & -.02 \\
\hline A & 289.1500 & 5.82681 & 5.82615 & .00066 & .011 & 7.677 & 7.604 & -.006 & -.00 \\
\hline A & 285.6500 & 5.42011 & 5.41960 & .00051 & .010 & 7.678 & 7.690 & -.012 & -.15 \\
\hline A & 285.6500 & 5.42003 & 5.41960 & .00043 & .008 & 7.678 & 7.689 & -.010 & -.13 \\
\hline A & 284.6500 & 5.30458 & 5.30419 & .00039 & .007 & 7.679 & 7.693 & -.014 & -.18 \\
\hline A & 284.6500 & 5.30469 & 5.30419 & .00050 & .009 & 7.679 & 7.697 & -.018 & -.23 \\
\hline A & 283.3500 & 5.18952 & 5.18925 & .00027 & .005 & 7.679 & 7.699 & -.019 & -.25 \\
\hline A & 283.1500 & 5.13217 & 5.13200 & .00017 & .003 & 7.679 & 7.702 & -.022 & -.29 \\
\hline A & 283.1500 & 5.13210 & 5.13200 & .00010 & .002 & 7.679 & 7.693 & -.013 & -.18 \\
\hline A & 282.6500 & 5.0750 .3 & 5.07494 & .00009 & .002 & 7.680 & 7.719 & -.039 & -.51 \\
\hline A & 282.6500 & 5.07495 & 5.07494 & .00001 & .000 & 7.680 & 7.685 & -.005 & -.07 \\
\hline A & 232.6500 & 5.07495 & 5.07494 & .00002 & .000 & 7.680 & 7.689 & -.010 & -.13 \\
\hline A & 282.5000 & 5.05789 & 5.05737 & .00002 & .000 & 7.680 & 7.698 & -.019 &.- .24 \\
\hline A & 303.1500 & 7.45336 & 7.45446 & -.00110 & -.015 & 7.622 & 7.619 & .003 & .04 \\
\hline A & 298.1500 & 6.86844 & 6.868373 & -.00029 & -.004 & 7.624 & 7.623 & .001 & .01 \\
\hline A & 298.1500 & 6.86837 & 6.86873 & -.00036 & -.005 & 7.624 & 7.622 & .001 & .02 \\
\hline A & 293.1500 & 6.28553 & 6.28525 & .00028 & .005 & 7.625 & 7.627 & -.002 & -.02 \\
\hline A & 289.1500 & 5.82149 & 5.82094 & .00055 & .009 & 7.627 & 7.632 & -.005 & -.07 \\
\hline A & 285.6500 & 5.41783 & 5.41742 & .00041 & .008 & 7.628 & 7.638 & -.010 & -.13 \\
\hline A & 284.6500 & 5.30310 & 5.30279 & .00031 & .006 & 7.628 & 7.640 & -.011 & -.15 \\
\hline A & 283.6500 & 5.18875 & 5.18855 & .00020 & .004 & 7.629 & 7.643 & -.015 & -.19 \\
\hline A & 283.1500 & 5.13169 & 5.13161 & .00008 & .002 & 7.629 & 7.640 & -.011 & -.14 \\
\hline A & 282.6500 & 5.07481 & 5.07482 & -.00001 & -.000 & 7.629 & 7.625 & .004 & .05 \\
\hline A & 303.1500 & 7.43832 & 7.43939 & -.00107 & -.014 & 7.583 & 7.580 & .003 & .04 \\
\hline A & 293.1500 & 0.85749 & 6.35782 & -.00033 & -.005 & 7.585 & 7.583 & .001 & .02 \\
\hline A & 298.1500 & 6.85754 & 6.85782 & -.00028 & -.004 & 7.585 & 7.584 & .001 & .01 \\
\hline A & 293.1500 & 6.27057 & 6.27828 & .00029 & .005 & 7.506 & 7.588 & -.002 & -.02 \\
\hline A & 289.1500 & 5.81749 & 5.81643 & .00056 & .010 & 7.588 & 7.593 & -.005 & -.07 \\
\hline A & 285.6500 & 5.41617 & 5.41574 & .00043 & .008 & 7.589 & 7.599 & -.010 & -.13 \\
\hline A & 284.6500 & 5.30193 & 5.30171 & .00027 & .005 & 7.589 & 7.599 & -.010 & -.13 \\
\hline A & 283.6500 & 5.18017 & 5.18801 & .00016 & .003 & 7.590 & 7.601 & -.012 & -.15 \\
\hline A & 283.1500 & 5.13139 & 5.13131 & .00008 & .002 & 7.590 & 7.600 & -.011 & -.14 \\
\hline A & 232.6500 & 5.07473 & 5.07473 & .00000 & .000 & 7.590 & 7.590 & -.000 & -.00 \\
\hline A & 303.1500 & 7.40673 & 7.40746 & -.00073 & -.010 & 7.499 & 7.497 & .002 & .03 \\
\hline A & 293.1500 & 6.83445 & 6.83468 & -.00023 & -.003 & 7.501 & 7.500 & .001 & .01 \\
\hline A & 293.1500 & 6.26 .383 & 6.26354 & .00029 & .005 & 7.503 & 7.505 & -.002 & -.02 \\
\hline A & 289.1500 & 5.80890 & 5.80344 & .00046 & .008 & 7.504 & 7.509 & -.005 & -.06 \\
\hline A & 285.6500 & 5.41252 & 5.41219 & .00033 & .006 & 7.506 & 7.513 & -.008 & -.10 \\
\hline$\hat{A}$ & $29+.6500$ & 5.29966 & 5.29941 & .00025 & .005 & 7.506 & 7.515 & -.009 & -.12 \\
\hline A & 283.6500 & 5.18690 & 5.18586 & .00012 & .002 & 7.506 & 7.515 & -.009 & -.12 \\
\hline A & 283.1500 & 5.13070 & 5.13067 & .00003 & .001 & 7.506 & 7.510 & -.004 & -.05 \\
\hline A & 282.6500 & 5.07449 & 5.07453 & -.00004 & -.001 & 7.507 & 7.483 & .019 & .25 \\
\hline A & 298.1334 & 6.53431 & 6.58350́ & .00075 & .011 & 6.580 & 6.532 & -.00 .3 & -.04 \\
\hline$\hat{H}$ & 290.1386 & 6.58443 & 6.53356 & .00037 & .013 & 6.580 & 6.583 & $\cdots .003$ & -.05 \\
\hline A & 273.1368 & 6.10011 & 6.09990 & .00021 & .003 & 6.551 & 6.582 & -.001 & -.02 \\
\hline A & $20 \% \cdot 1390$ & 5.71065 & 5.71073 & -.00013 & .002 & 5.533 & 6.581 & .001 & .02 \\
\hline A & $285 \cdot 6.571$ & 5.36 .25 & 5.30758 & -.000 .33 & -.006 & 6.534 & 6.578 & .008 & .09 \\
\hline A & 203.6392 & 5.10009 & 5.16922 & -.00033 & -.006 & 6.5134 & 6.572 & .012 & .19 \\
\hline A & $=82.3333$ & 5.0 .3721 & $5.037 \div 1$ & -.00030 & $-.00 \dot{c}$ & u. 585 & 6.543 & .041 & .83 \\
\hline A & 282.2193 & 5.02516 & 5.02580 & -.00054 & -.013 & 6.585 & 3.470 & .105 & 1.61 \\
\hline
\end{tabular}


Table BI. (Continued)

\begin{tabular}{|c|c|c|c|c|c|c|c|c|c|}
\hline \multirow[t]{3}{*}{ EXF', II } & TEMFEFIATUFE & \multicolumn{4}{|c|}{ FFIESSUFE } & \multicolumn{4}{|c|}{ LIENSITY } \\
\hline & \multirow[t]{2}{*}{$\kappa$} & \multicolumn{4}{|c|}{ MFA } & \multicolumn{4}{|c|}{ MOL/DM3 } \\
\hline & & $E \times F$ & CALC & IIFF & $\%$ LIIFF & EXF & CALC & IIIFF & $\%$ WIFF \\
\hline A & 282.1893 & 5.02186 & 5.02270 & -.00084 & -.017 & 6.585 & 6.443 & .142 & 2.16 \\
\hline A & 202.0893 & 5.01081 & 5.01150 & -.00069 & -.014 & 6.585 & 6.322 & .263 & 3.99 \\
\hline A & 293.1386 & $6.386,68$ & 6.38502 & .00166 & .026 & 5.899 & 5.904 & -.005 & -.09 \\
\hline A & 298.1336 & 6.38663 & 6.38502 & .00161 & .025 & 5.899 & 5.904 & -.005 & -.09 \\
\hline$A$ & 282.3333 & 5.02445 & 5.02435 & -.00020 & -.004 & 5.903 & 5.898 & .005 & .09 \\
\hline A & 202.0393 & 4.99780 & 4.99800 & -.00020 & -.004 & 5.903 & 5.897 & .006 & .11 \\
\hline A & 231.6893 & 4.95445 & 4.96603 & -.00158 & -.032 & 5.904 & 5.842 & .062 & 1.04 \\
\hline E & 298.1385 & 8.39404 & 8.39185 & .00219 & .026 & 10.617 & 10.619 & -.002 & -.02 \\
\hline E & 298.1386 & 8.39409 & 8.37185 & .00224 & .027 & 10.617 & 10.620 & -.002 & -.02 \\
\hline $\mathrm{E}$ & 280.0394 & 4.84374 & 4.84224 & .00150 & .031 & 10.626 & 10.633 & -.006 & -.06 \\
\hline $\mathrm{E}$ & 279.5394 & 4.75176 & 4.75086 & .00090 & .019 & 10.627 & 10.631 & -.004 & -.04 \\
\hline$B$ & 298.1306 & 7.22930 & 7.23118 & -.00188 & -.026 & 8.750 & 8.745 & .005 & .06 \\
\hline E & 298.1386 & 7.22931 & 7.23118 & -.00187 & -.026 & 8.750 & 8.745 & .005 & .06 \\
\hline $\mathrm{H}$ & 282.3593 & 5.04648 & 5.04602 & .00046 & .009 & 8.757 & 8.794 & -.037 & -.42 \\
\hline E & 282.3333 & 5.04177 & 5.04143 & .00034 & .007 & 8.757 & 8.786 & -.030 & -.34 \\
\hline E & 282.2143 & 5.02663 & 5.02633 & .00030 & .006 & 8.757 & 8.791 & -.034 & -.39 \\
\hline E & 282.1993 & 5.02345 & 5.02317 & .00028 & .006 & 8.757 & 8.791 & -.034 & -.39 \\
\hline $\mathrm{E}$ & 282.1893 & 5.02348 & 5.02317 & .00031 & .006 & 8.757 & 8.794 & -.038 & -.43 \\
\hline E & 282.1893 & 5.02345 & 5.02317 & .00020 & .006 & 8.757 & 8.791 & -.034 & -.39 \\
\hline E & 303.1500 & 7.44551 & 7.44692 & -.00141 & -.019 & 7.602 & 7.599 & .004 & .05 \\
\hline H & 293.1500 & 6.86299 & 6.85327 & -.00028 & -.004 & 7.604 & 7.603 & .001 & .01 \\
\hline E & 298.1500 & 6.86292 & 6.86327 & -.00035 & -.005 & 7.604 & 7.603 & .001 & .02 \\
\hline $\mathrm{H}$ & 293.1500 & 6.28229 & 6.28176 & .00053 & .008 & 7.606 & 7.609 & -.003 & -.04 \\
\hline B & 289.1500 & 5.81975 & 5.81893 & .00082 & .014 & 7.507 & 7.615 & -.008 & -.10 \\
\hline B & 285.6500 & 5.41734 & 5.41658 & .00076 & .014 & 7.609 & 7.626 & -.018 & -.23 \\
\hline E & 284.6500 & 5.30307 & 5.30225 & .00082 & .016 & 7.609 & 7.639 & -.030 & -.39 \\
\hline $\mathrm{B}$ & 283.6500 & 5.18886 & 5.18828 & .00058 & .011 & 7.609 & 7.651 & -.042 & -.55 \\
\hline E & 283.1500 & 5.13189 & 5.13146 & .00043 & .008 & 7.609 & 7.666 & -.056 & -.74 \\
\hline E & 298.1386 & 6.56543 & 6.56455 & .00088 & .013 & 6.511 & 6.514 & -.003 & -.05 \\
\hline E & 298.1385 & 6.56551 & 6.56455 & .00096 & .015 & 6.511 & 6.515 & -.003 & -.05 \\
\hline $\mathrm{E}$ & 293.1388 & 6.08762 & 6.08714 & .00048 & .008 & 6.513 & 6.515 & -.003 & -.04 \\
\hline E & 289.1390 & 5.70295 & 5.70280 & .00015 & .003 & 6.514 & 6.515 & -.001 & -.02 \\
\hline E & 285.6391 & 5.36362 & 5.36356 & .00006 & .001 & 6.515 & 6.516 & -.001 & -.02 \\
\hline E & $283.63 \% 2$ & 5.16741 & 5.16734 & .00007 & .001 & 6.516 & 6.518 & -.002 & -.04 \\
\hline E & 282.3333 & 5.03714 & 5.03690 & .00015 & .003 & 6.516 & 6.534 & -.018 & -.28 \\
\hline $\mathrm{H}$ & 282.2393 & 5.02761 & 5.02745 & .00016 & .003 & 6.516 & 6.540 & -.024 & -.37 \\
\hline E & 282.1893 & 5.02242 & 5.02230 & .00005 & .001 & 6.516 & 6.526 & -.010 & -.16 \\
\hline B & 298.1386 & 6.38518 & 0.38351 & .00167 & .026 & 5.894 & 5.900 & -.005 & -.09 \\
\hline E & 293.1386 & 6.38512 & 6.3835 .11 & .00161 & .025 & 5.894 & 5.099 & -.005 & -.09 \\
\hline E & 293.1388 & 5.95215 & 5.96127 & .00088 & .015 & 5.896 & 5.899 & -.004 & -.07 \\
\hline E & 289.1390 & 5.62038 & 5.31984 & .00054 & .010 & 5.897 & 5.900 & -.004 & -.06 \\
\hline$E$ & 285.6391 & 5.31734 & 5.31702 & .00032 & .006 & 5.899 & 5.901 & -.003 & -.06 \\
\hline EI & 283.6392 & 5.14149 & 5.14121 & .00023 & .006 & 5.890 & 5.903 & -.005 & -.08 \\
\hline B & 282.3333 & 5.02474 & 5.02447 & .00027 & .005 & 5.898 & 5.906 & -.007 & -.13 \\
\hline E & 282.2393 & $5.01 \leq 24$ & 5.01598 & .00026 & .005 & 5.899 & 5.906 & -.008 & -.13 \\
\hline E & 282.1393 & $5.00>20$ & 5.00692 & .00028 & .005 & 5.899 & 5.907 & -.008 & -.14 \\
\hline E & 281.9393 & 4.98901 & 4.98875 & .00025 & .005 & 5.899 & 5.907 & -.009 & -.15 \\
\hline E & 201.3393 & 4.97992 & 4.97964 & .00028 & .006 & 5.899 & 5.909 & -.011 & -.18 \\
\hline E & 281.7393 & 4.97071 & 4.97049 & .00022 & .004 & 5.899 & 5.907 & -.009 & -.15 \\
\hline $\mathrm{E}$ & 281.6893 & 4.96579 & 4.95591 & -.00012 & -.002 & 5.899 & 5.894 & .005 & .09 \\
\hline E & 281.6373 & 4.96035 & 4.96132 & -.00097 & -.020 & 5.899 & 5.853 & .040 & .68 \\
\hline
\end{tabular}


TABLE BI. (Continued)

\begin{tabular}{|c|c|c|c|c|c|c|c|c|c|}
\hline \multirow[t]{3}{*}{ EXF' • I I I } & TEMFERATUKE & \multicolumn{4}{|c|}{ FRESSURE } & \multicolumn{4}{|c|}{ DENSITY } \\
\hline & \multirow[t]{2}{*}{$\kappa$} & \multicolumn{4}{|c|}{ MF'A } & \multicolumn{4}{|c|}{ MOL/IMB } \\
\hline & & EXF & CALC & IIFF & XIIIFF & EXF & CALC & UIFF & XIIIFF \\
\hline 1 & 282.3500 & 5.04197 & 5.04084 & .00113 & .022 & 7.635 & 8.563 & -.928 & -12.15 \\
\hline 1 & 282.3600 & 5.04288 & 5.04197 & .00091 & .018 & 7.635 & 8.499 & -.864 & -11.32 \\
\hline 1 & 282.3700 & 5.04403 & 5.04311 & .00092 & .018 & 7.635 & 8.483 & -.848 & $-11,11$ \\
\hline 1 & 282.3800 & 5.04518 & 5.04424 & .00094 & .019 & 7.635 & 8.467 & -.832 & -10.90 \\
\hline 1 & 282.3900 & 5.04626 & 5.04537 & .00089 & .018 & 7.635 & 8.433 & -.798 & -10.46 \\
\hline 1 & 282.4000 & 5.04744 & 5.04650 & .00094 & .019 & 7.635 & 8.425 & -.790 & -10.34 \\
\hline 1 & 282.4100 & 5.04858 & 5.04763 & .00095 & .019 & 7.635 & 8.406 & -.771 & -10.10 \\
\hline 1 & 282.4200 & 5.04978 & 5.04876 & .00102 & .020 & 7.635 & 8.403 & -.768 & -10.06 \\
\hline 1 & 282.4300 & 5.05083 & 5.04990 & .00093 & .018 & 7.635 & 8.360 & -.725 & -9.50 \\
\hline 1 & 282.4500 & 5.05324 & 5.05216 & .00108 & .021 & 7.635 & 8.359 & -.724 & -9.49 \\
\hline 1 & 282.5500 & 5.06452 & 5.06349 & .00103 & .020 & 7.635 & 8.159 & -.524 & -6.87 \\
\hline 1 & 282.6500 & 5.07586 & 5.07483 & .00103 & .020 & 7.635 & 8.019 & -.384 & -5.02 \\
\hline 1 & 282.7500 & 5.08723 & 5.08618 & .00105 & .021 & 7.635 & 7.930 & -.295 & -3.86 \\
\hline 1 & 282.8500 & 5.09858 & 5.09754 & .00104 & .020 & 7.635 & 7.865 & -.230 & -3.01 \\
\hline 1 & 282.9500 & 5.10994 & 5.10890 & .00104 & .020 & 7.635 & 7.821 & -.186 & -2.44 \\
\hline 1 & 283,0500 & 5.12136 & 5.12028 & .00108 & .021 & 7.635 & 7.798 & -.163 & -2.13 \\
\hline 1 & 283.1500 & 5.13277 & 5.13165 & .00112 & .022 & 7.635 & 7.778 & -.143 & -1.87 \\
\hline 1 & 283.3500 & 5.15567 & 5.15443 & .00124 & .024 & 7.635 & 7.757 & -.122 & -1.59 \\
\hline 1 & 284.1500 & 5.24722 & 5.24574 & .00148 & .028 & 7.635 & 7.706 & -.071 & -.93 \\
\hline 1 & 285.1500 & 5.36197 & 5.36030 & .00167 & .031 & 7.635 & 7.682 & -.047 & -.62 \\
\hline 1 & 286.1500 & 5.47701 & 5.47522 & .00179 & .033 & 7.635 & 7.670 & -.035 & -.46 \\
\hline 1 & 287.1500 & 5.59247 & 5.59047 & .00200 & .036 & 7.635 & 7.664 & -.029 & -.39 \\
\hline 1 & 288,1500 & 5.70808 & 5.70600 & .00208 & .036 & 7.635 & 7.659 & -.024 & -.32 \\
\hline 1 & 293.1500 & 6.28886 & 6.28697 & .00189 & .030 & 7.635 & 7.646 & -.011 & -.14 \\
\hline 1 & 298.1500 & 6.87365 & 6.87194 & .00171 & .025 & 7.635 & 7.641 & -.006 & -.08 \\
\hline 1 & 303,1500 & 7.46149 & 7.45959 & .00190 & .025 & 7.635 & 7.640 & -.005 & -.06 \\
\hline 1 & 303.1500 & 6.84890 & 6.84568 & .00322 & .047 & 6.000 & 6.008 & -.008 & -.14 \\
\hline 1 & 303.1500 & 7.03963 & 7.03704 & .00259 & .037 & 6.500 & 6.507 & -.007 & -.11 \\
\hline 1 & 303,1500 & 7.22319 & 7.22142 & .00177 & .025 & 7.000 & 7.005 & -.005 & -.07 \\
\hline 1 & 303.1500 & 7.40872 & 7.40769 & .00103 & .014 & 7.500 & 7.503 & -.003 & -.04 \\
\hline 1 & 303.1500 & 7.60625 & 7.60600 & .00025 & .003 & 8.000 & 8.001 & -.001 & -.01 \\
\hline 1 & 303.1500 & 7.82674 & 7.82818 & -.00144 & -.018 & 8.500 & 8.497 & .003 & .04 \\
\hline 1 & 303.1500 & 8.08490 & 8.08845 & -.00355 & -.044 & 9.000 & 8.994 & .006 & .07 \\
\hline 1 & 303.1500 & 8.39804 & 8.40388 & -.00584 & -.069 & 9.500 & 9.492 & .008 & .09 \\
\hline 1 & 303.1500 & 8.79026 & 8.79482 & -.00456 & -.052 & 10.000 & 9.995 & .005 & .05 \\
\hline 1 & 303.1500 & 9.28873 & 9.28491 & .00382 & .041 & 10.500 & 10.503 & -.003 & -.03 \\
\hline 1 & 298.1500 & 6.42028 & 6.41712 & .00316 & .049 & 6.000 & 6.010 & -.010 & -.17 \\
\hline 1 & 298.1500 & 6.56526 & 6.56251 & .00275 & .042 & 6.500 & 6.510 & -.010 & -.15 \\
\hline 1 & 298.1500 & 6.70124 & 6.69887 & .00237 & .035 & 7.000 & 7.009 & -.009 & -.13 \\
\hline 1 & 298.1500 & 6.83652 & 6.83436 & .00216 & .032 & 7.500 & 7.508 & -.008 & -.10 \\
\hline 1 & 298.1500 & 6.98029 & 6.97806 & .00223 & .032 & 8.000 & 8.007 & -.007 & -.09 \\
\hline 1 & 298.1500 & 7.14174 & 7.14057 & .00117 & .016 & 8.500 & 8.503 & -.003 & -.04 \\
\hline 1 & 298.1500 & 7.33468 & 7.33480 & -.00012 & -.002 & 9.000 & 9.000 & .000 & .00 \\
\hline 1 & 298.1500 & 7.57508 & 7.57669 & -.00161 & -.021 & 9.500 & 9.497 & .003 & .03 \\
\hline 1 & 298.1500 & 7.88281 & 7.88568 & -.00287 & -.036 & 10.000 & 9.996 & .004 & .04 \\
\hline 1 & 298.1500 & 8.29048 & 8.28482 & .00566 & .068 & 10.500 & 10.506 & -.006 & -.06 \\
\hline 1 & 293.1500 & 5.98672 & 5.98523 & .00149 & .025 & 6.000 & 6.007 & -.007 & -.12 \\
\hline 1 & 293.1500 & 6.08774 & 6.08581 & .00193 & .032 & 6.500 & 6.510 & -.010 & -.16 \\
\hline 1 & 293.1500 & 6.17782 & 6.17598 & .00184 & .030 & 7.000 & 7.011 & -.011 & -.15 \\
\hline 1 & 293.1500 & 6.26526 & 6.26302 & .00224 & .036 & 7.500 & 7.513 & -.013 & -.17 \\
\hline 1 & 293.1500 & 6.35743 & 6.35471 & .00272 & .043 & 8.000 & 8.014 & -.014 & -.17 \\
\hline 1 & 293.1500 & 6.46250 & 6.46012 & .00238 & .037 & 8.500 & 8.510 & -.010 & -.12 \\
\hline 1 & 293.1500 & 6.59227 & 6.59055 & .00172 & .026 & 9.000 & 9.006 & -.006 & -.06 \\
\hline 1 & 293.1500 & 6.76196 & 6.76051 & .00145 & .021 & 9.500 & 9.504 & -.004 & -.04 \\
\hline 1 & 293.1500 & 6.99048 & 6.98849 & .00199 & .028 & 10.000 & 10,004 & -.004 & -.04 \\
\hline 1 & 293.1500 & 7.30284 & 7.29694 & .00590 & .081 & 10.500 & 10.508 & -.008 & -.08 \\
\hline
\end{tabular}


Table BI. (Continued)

F'RESSURE

MFA

\section{IIENSITY}

MOL/IIMJ

\begin{tabular}{|c|c|c|c|c|c|c|c|c|c|c|}
\hline & & EXF & CALC & IIIFF & \#IIFF & EXF & CALC & IIIFF & \%[IIFF & \\
\hline 1 & $288 \cdot 1500$ & 5.54964 & 5.54851 & .00113 & .020 & 6.000 & 6.009 & -.009 & -.15 & \\
\hline 1 & 288.1500 & 5.60692 & 5.60585 & .00107 & .019 & 6.500 & 6.511 & -.011 & -.16 & \\
\hline $\begin{array}{l}1 \\
1\end{array}$ & $\begin{array}{l}288 \cdot 1500 \\
288 \cdot 1500\end{array}$ & $\begin{array}{l}5.65366 \\
5.69652\end{array}$ & $\begin{array}{l}5.65247 \\
5.69461\end{array}$ & $\begin{array}{l}.00119 \\
.00191\end{array}$ & $\begin{array}{l}.021 \\
.033\end{array}$ & $\begin{array}{l}7.000 \\
7.500\end{array}$ & $\begin{array}{l}7.014 \\
7.523\end{array}$ & $\begin{array}{l}-.014 \\
-.023\end{array}$ & $\begin{array}{l}-.20 \\
-.30\end{array}$ & \\
\hline 1 & $288+1500$ & 5.74088 & 5.73834 & .00254 & .044 & 8.000 & 8.027 & -.027 & -.34 & \\
\hline 1 & $288 \cdot 1500$ & 5.79342 & 5.79053 & .00289 & .050 & 8.500 & 8.524 & -.024 & -.28 & \\
\hline 1 & 288.1500 & 5.86313 & 5.86034 & .00279 & .048 & 9.000 & 9.017 & -.017 & -.19 & \\
\hline 1 & $288+1500$ & 5.96313 & 5.96052 & .00261 & .044 & 9.500 & 9.511 & -.011 & -.11 & 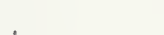 \\
\hline 1 & 288.1500 & 6.11098 & 6.10843 & .00255 & .042 & 10.000 & 10.007 & -.007 & -.07 & \\
\hline 1 & $288 \cdot 1500$ & 6.33296 & 6.32614 & .00682 & .108 & 10.500 & 10.513 & -.013 & -.12 & \\
\hline 1 & $288 \cdot 1500$ & 5.67503 & 5.67373 & .00130 & .023 & 7.250 & 7.266 & -.016 & -.21 & \\
\hline 1 & $288 \cdot 1500$ & 5.63106 & 5.63009 & .00097 & .017 & 6.750 & 6.760 & -.010 & -.15 & \\
\hline 1 & 284.1500 & 5.57352 & 5.56745 & .00607 & .109 & 10.500 & 10.516 & -.016 & -.16 & \\
\hline 1 & $284 \cdot 1500$ & 5.42568 & 5.42329 & .00239 & .044 & 10.000 & 10.011 & -.011 & -.11 & \\
\hline 1 & 284.1500 & 5.34153 & 5.33931 & .00222 & .042 & 9.500 & 9.518 & -.018 & -.19 & \\
\hline 1 & 284.1500 & 5.29548 & 5.29321 & .00227 & .043 & 9.000 & 9.033 & -.033 & -.37 & \\
\hline 1 & $284 \cdot 1500$ & 5.27042 & 5.26832 & .00210 & .040 & 8.500 & 8.555 & -.055 & -.65 & \\
\hline 1 & 284.1500 & 5.25551 & 5.25371 & .00180 & .034 & 8.000 & 8.074 & -.074 & -.92 & \\
\hline 1 & $284+1500$ & 5.24964 & 5.24814 & .00150 & .029 & 7.750 & 7.820 & -.070 & -.90 & \\
\hline 1 & $284 \cdot 1500$ & 5.24427 & 5.24298 & .00129 & .025 & 7.500 & 7.563 & -.063 & -.84 & \\
\hline 1 & $284 \cdot 1500$ & 5.23885 & 5.23777 & .00108 & .021 & 7.250 & 7.301 & -.051 & -.70 & \\
\hline 1 & 284.1500 & 5.23306 & 5.23207 & .00099 & .019 & 7.000 & 7.041 & -.041 & -.59 & \\
\hline 1 & 284.1500 & 5.22627 & 5.22539 & .00088 & .017 & 6.750 & 6.780 & -.030 & -.45 & \\
\hline 1 & 284.1500 & 5.21797 & 5.21715 & .00082 & .016 & 6.500 & 6.522 & -.022 & -.34 & \\
\hline 1 & 284.1500 & 5.19400 & 5.19304 & .00096 & .018 & 6.000 & 6.016 & -.016 & -.26 & \\
\hline 1 & $284 \cdot 1500$ & 5.15364 & 5.15266 & .00098 & .019 & 5.500 & 5.510 & -.010 & -.17 & \\
\hline 1 & $283+1500$ & 5.10345 & 5.10259 & .00086 & .017 & 6.000 & 6.020 & -.020 & -.33 & \\
\hline 1 & $283 \cdot 1500$ & 5.11928 & 5.11850 & .00078 & .015 & 6.500 & 6.537 & -.037 & -.57 & \\
\hline 1 & 283.1500 & 5.12707 & 5.12620 & .00087 & .017 & 7.000 & 7.083 & -.083 & -1.19 & \\
\hline 1 & $283+1500$ & 5.13169 & 5.13062 & .00107 & .021 & 7.500 & 7.640 & -.140 & -1.86 & \\
\hline 1 & $283+1500$ & 5.13594 & 5.13469 & .00125 & .024 & 8.000 & 8.125 & -.125 & -1.56 & \\
\hline 1 & 283.1500 & 5.14271 & $5+14122$ & .00149 & .029 & 8.500 & 8.576 & -.076 & -.90 & \\
\hline 1 & 283.1500 & 5.15732 & 5.15556 & .00176 & .034 & 9.000 & 9.040 & -.040 & -.44 & \\
\hline 1 & $283 \cdot 1500$ & 5.18994 & 5.18820 & .00174 & .033 & 9.500 & 9.518 & -.018 & -.19 & \\
\hline 1 & 283.1500 & 5.25792 & 5.25591 & .00201 & .038 & 10.000 & 10.011 & -.011 & -.11 & \\
\hline 1 & 283.1500 & 5.38690 & 5.38124 & .00566 & .105 & 10.500 & 10.517 & -.017 & -.16 & \\
\hline 1 & 283.1500 & 5.13368 & 5.13255 & .00113 & .022 & 7.750 & 7.888 & -.138 & -1.78 & \\
\hline 1 & 283.1500 & 5.12950 & 5.12861 & .00089 & .017 & 7.250 & 7.357 & -.107 & -1.47 & \\
\hline 1 & $283 \cdot 1500$ & $5+12379$ & $5+12301$ & .00078 & .015 & 6.750 & 6.804 & -.054 & -.80 & \\
\hline 1 & 283.3500 & 5.18470 & 5.18289 & .00181 & .035 & 9.000 & 9.037 & -.037 & -.41 & \\
\hline 1 & 283.3500 & $5+16820$ & $5+16646$ & .00174 & .034 & 8.500 & 8.575 & -.075 & -.88 & \\
\hline 1 & 283.3500 & 5.15981 & 5.15838 & .00143 & .028 & 8.000 & 8.112 & -.112 & -1.40 & \\
\hline 1 & 283.3500 & 5.15692 & $5+15560$ & .00132 & .026 & 7.750 & 7.874 & -.124 & -1.59 & \\
\hline 1 & 283.3500 & 5.15419 & 5.15308 & .00111 & .022 & 7.500 & 7.611 & -.111 & -1.48 & \\
\hline 1 & 283.3500 & 5.15144 & $5+15047$ & .00097 & .019 & 7.250 & 7.340 & -.090 & $-1 \cdot 25$ & \\
\hline 1 & 283.3500 & 5.14829 & 5.14743 & .00086 & .017 & 7.000 & 7.065 & -.065 & -.93 & \\
\hline 1 & 283.3500 & 5.14440 & $5+14356$ & .00084 & .016 & 6.750 & 6.798 & -.048 & -.71 & \\
\hline 1 & 283.3500 & 5.13908 & 5.13832 & .00076 & .015 & 6.500 & 6.532 & -.032 & -.49 & \\
\hline 1 & 283.3500 & 5.12159 & 5.12076 & .00083 & .016 & 6.000 & 6.018 & -.018 & -.29 & \\
\hline 1 & 283.3500 & 5.08842 & 5.08745 & .00097 & .019 & 5.500 & 5.511 & -.011 & -.20 & \\
\hline 1 & 282.5500 & 5.06449 & 5.06351 & .00098 & .019 & 7.650 & 8.150 & -.500 & -6.53 & \\
\hline 1 & 282.5500 & 5.06446 & 5.06344 & .00102 & .020 & 7.600 & 8.140 & -.540 & -7.10 & \\
\hline 1 & 282.6500 & 5.08928 & 5.08779 & .00149 & .029 & 9.000 & 9.047 & -.047 & -.52 & \\
\hline 1 & $282 \cdot 6500$ & 5.07993 & 5.07866 & .00127 & .025 & 8.500 & 8.615 & -.115 & $-1 \cdot 35$ & \\
\hline 1 & 282.6500 & 5.07695 & 5.07580 & .00115 & .023 & 8.000 & 8.270 & -.270 & -3.37 & \\
\hline 1 & 282.6500 & 5.07616 & 5.07511 & .00105 & .021 & 7.750 & 8.101 & -.351 & $-4 \cdot 53$ & \\
\hline
\end{tabular}


Table BI. (Continued)

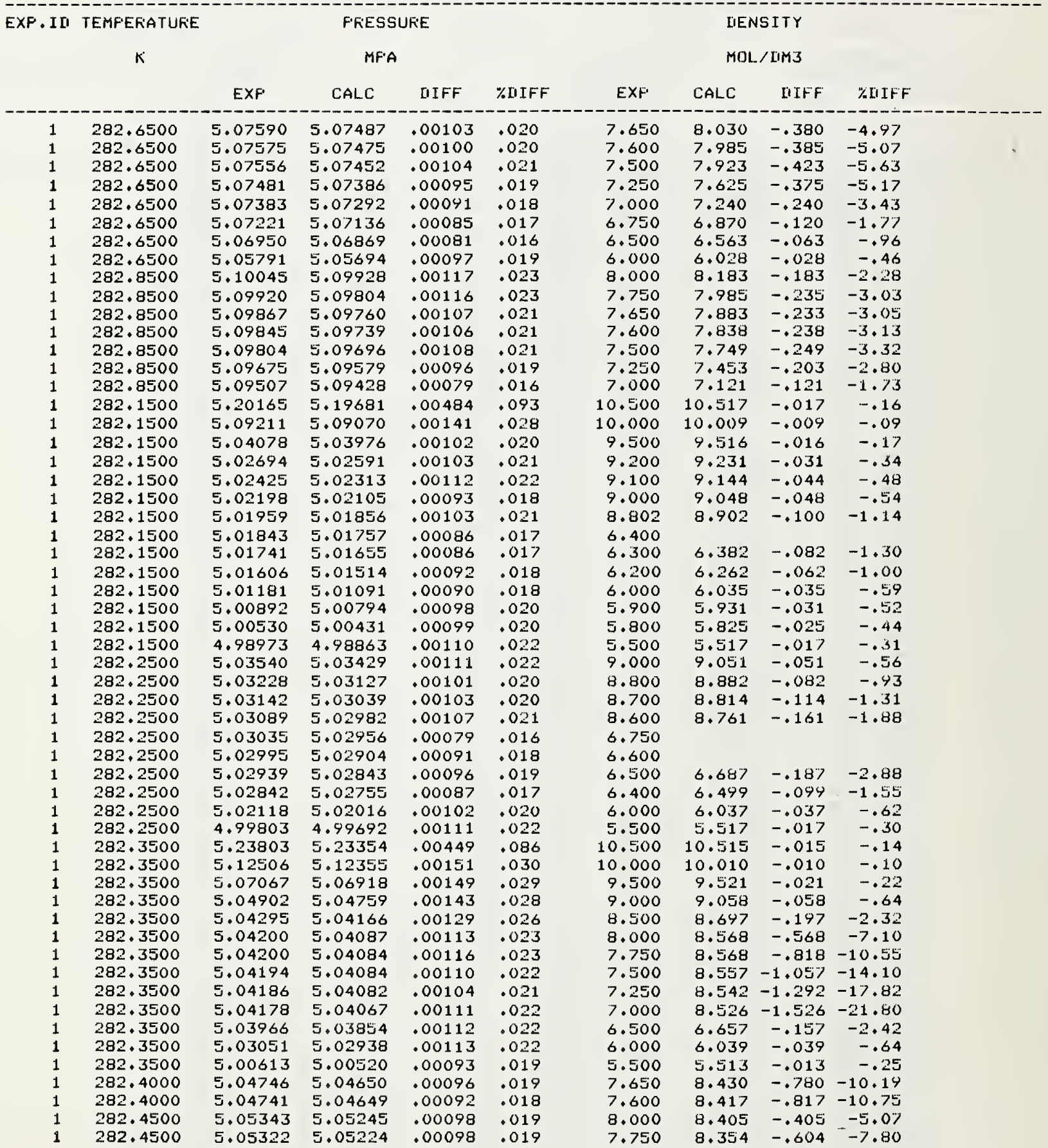


Table BI. (Continued)

EXF. ID TEMF'EFATUF'E

$\kappa$

\section{F'FESSUFE}

MF'A

\section{IIENSTTY}

$\mathrm{MOL} /[\mathrm{IM} 3$

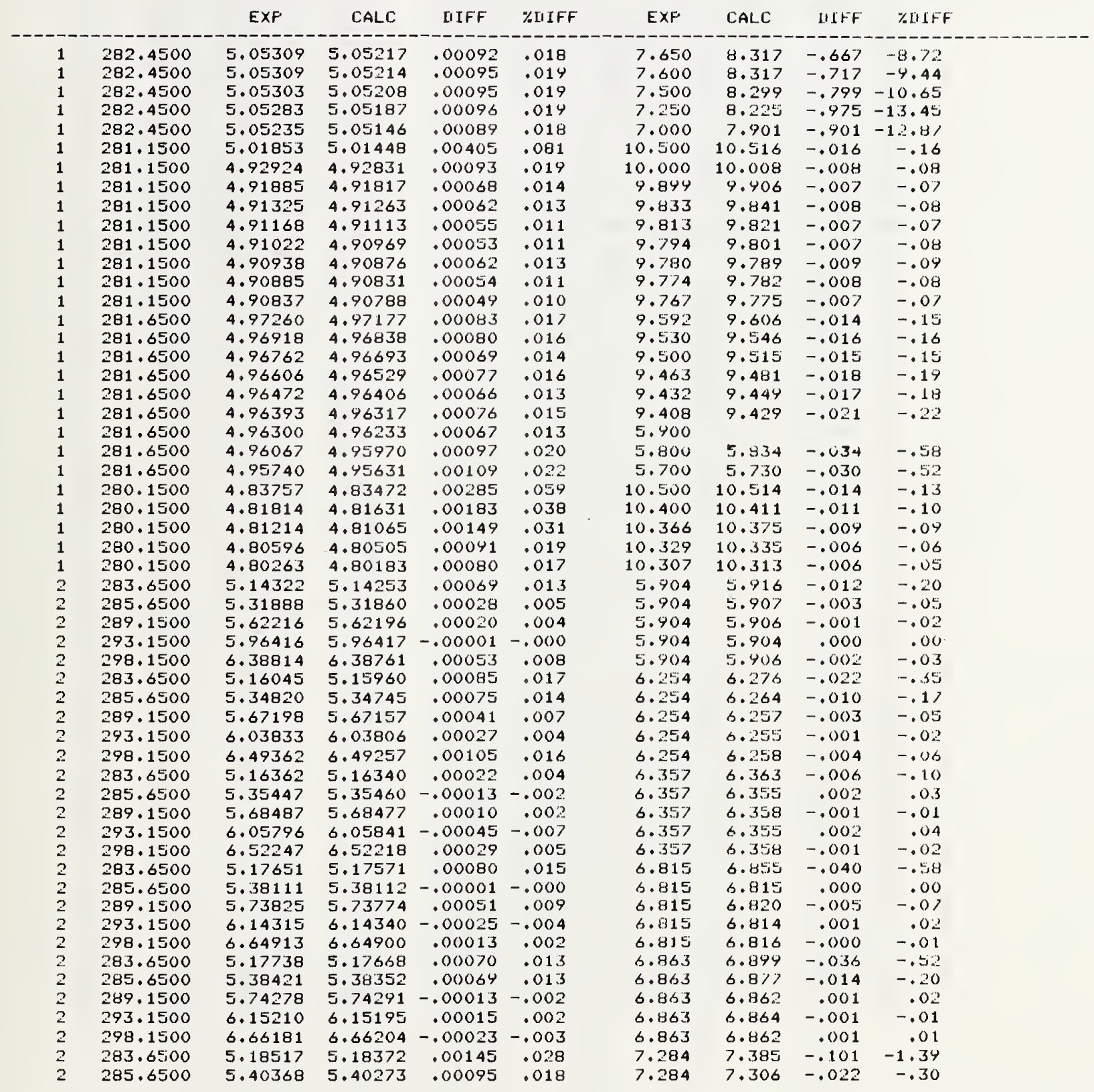


Table BI. (Continued)

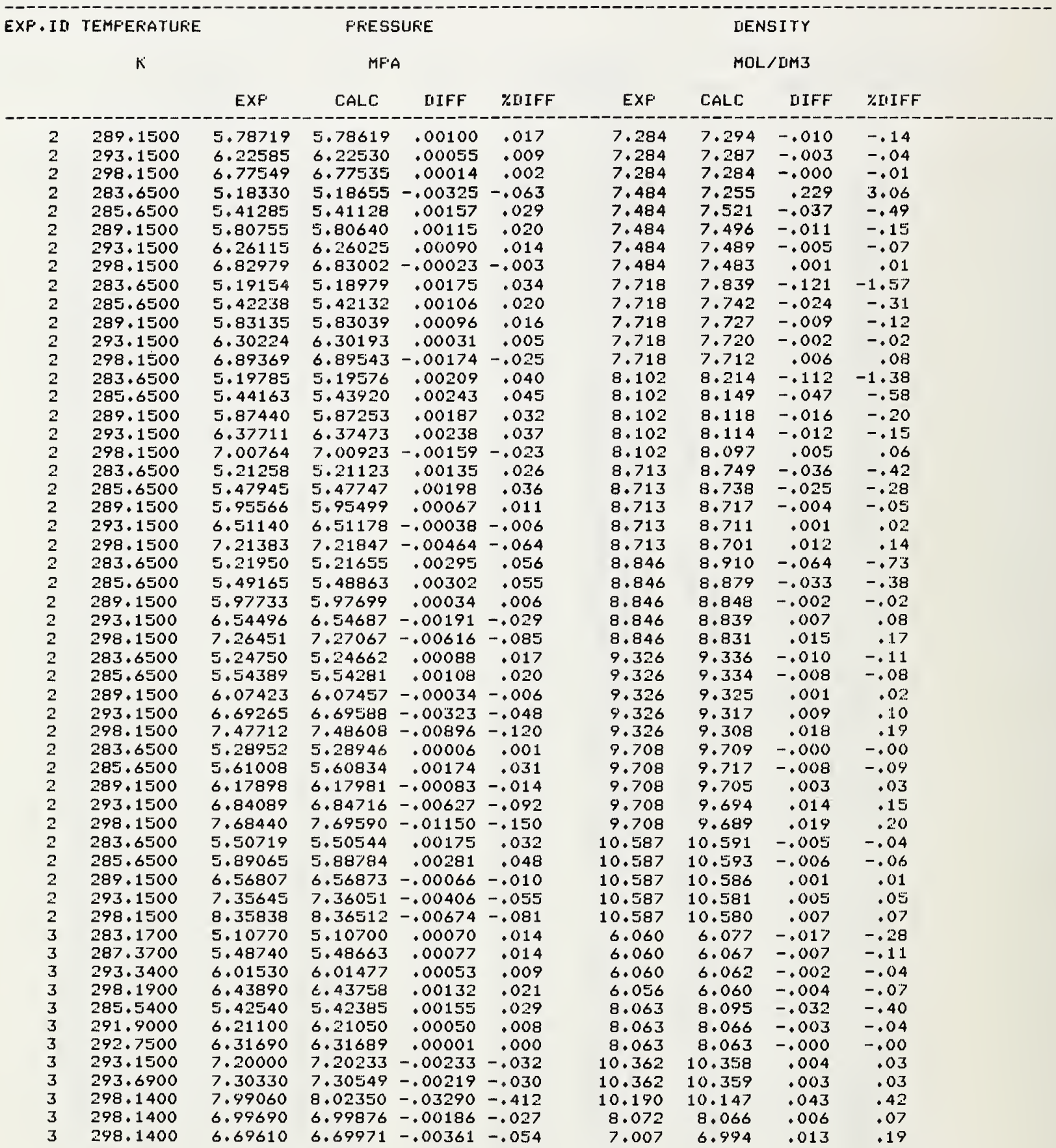


Table BII. Comparison of experimental saturation pressures with the present formulation.

\begin{tabular}{|c|c|c|c|c|c|c|}
\hline \multirow[t]{2}{*}{$\begin{array}{l}\text { EXF' } \\
\text { I II. }\end{array}$} & \multirow[t]{2}{*}{$\begin{array}{c}\text { TEMFEERATUF'E } \\
\qquad K\end{array}$} & \multicolumn{4}{|c|}{$\begin{array}{l}\text { F'KESSSUFIE } \\
\text { MF'A }\end{array}$} & \multirow[t]{2}{*}{$\begin{array}{l}\text { IENSITY } \\
\text { MOL/IIM3 }\end{array}$} \\
\hline & & $E \times F$ & CALC & IIIFF & \%IIIFF & \\
\hline 1 & 279.1500 & 4.69367 & 4.69366 & .00001 & .0002 & \\
\hline 1 & 280.1500 & 4.79973 & 4.79939 & .00034 & .007 & \\
\hline 1 & $281 \cdot 1500$ & 4.90813 & 4.90753 & .00006 & .012 & \\
\hline 1 & 281.6500 & 4.96331 & 4.96256 & .00075 & .015 & \\
\hline 1 & $282 \cdot 1500$ & 5.01915 & 5.01831 & .00084 & .017 & \\
\hline 1 & 282.2000 & 5.02481 & 5.02393 & .00088 & .018 & \\
\hline 1 & 282.2500 & 5.03046 & 5.02956 & .00090 & .018 & \\
\hline 1 & 282.3000 & 5.03612 & 5.03519 & .00093 & .018 & \\
\hline$A$ & 279.4394 & 4.72372 & 4.72402 & -.00030 & -.006 & 10.552 \\
\hline$A$ & 280.6394 & 4.85179 & 4.85200 & -.00021 & -.004 & 10.032 \\
\hline A & 280.5894 & 4.84640 & 4.84660 & -.00020 & -.004 & 10.032 \\
\hline$A$ & 280.5844 & 4.84596 & 4.84606 & -.00010 & -.002 & 10.032 \\
\hline$A$ & 281.7393 & 4.97264 & 4.97246 & .00013 & .004 & 9.293 \\
\hline$A$ & 281.6893 & 4.96707 & 4.96692 & .00015 & .003 & 9.293 \\
\hline$A$ & 281.7393 & 4.97271 & 4.97246 & .00025 & .005 & 9.293 \\
\hline$A$ & 282.0693 & 5.00948 & 5.00933 & .00015 & .003 & 8.917 \\
\hline$A$ & 282.0733 & 5.00997 & 5.00985 & .00012 & .002 & 8.917 \\
\hline$A$ & 282.0493 & 5.00729 & 5.00702 & .00027 & .005 & 8.917 \\
\hline$A$ & 282.1632 & 5.02002 & 5.01994 & .00008 & .002 & 8.764 \\
\hline$A$ & $282 \cdot 1380$ & 5.01718 & 5.01696 & .00022 & .004 & 8.764 \\
\hline$A$ & 281.1500 & 4.90746 & 4.90753 & -.00007 & -.001 & 7.680 \\
\hline$A$ & 282.0500 & 5.00720 & 5.00710 & .00010 & .002 & 7.580 \\
\hline$A$ & 281.7500 & 4.97377 & 4.97365 & .00012 & .002 & 7.680 \\
\hline$A$ & 281.4500 & 4.94055 & 4.94047 & .00008 & .002 & 7.630 \\
\hline$A$ & 281.7500 & 4.97373 & 4.97365 & .00008 & .002 & 7.680 \\
\hline$A$ & 282.3440 & 5.04018 & 5.04016 & .00002 & .000 & 7.680 \\
\hline$A$ & 282.3440 & 5.0401 .9 & 5.04016 & .00003 & .001 & 7.630 \\
\hline$A$ & 282.3440 & 5.04024 & 5.04016 & .00008 & .002 & 7.630 \\
\hline$A$ & 281.7500 & 4.97366 & 4.97365 & .00001 & .000 & 7.629 \\
\hline$A$ & 282.3440 & 5.04007 & 5.04016 & -.00009 & -.002 & 7.629 \\
\hline$A$ & 281.7500 & 4.97366 & 4.97365 & .00001 & .000 & 7.590 \\
\hline A & 281.7500 & 4.97365 & 4.97365 & -.00000 & -.000 & 7.590 \\
\hline$A$ & 282.3440 & 5.04016 & 5.04016 & .00000 & .000 & 7.590 \\
\hline$A$ & 282.3440 & 5.04011 & 5.04016 & -.00005 & -.001 & 7.507 \\
\hline$A$ & 280.6394 & 4.85177 & 4.85200 & -.00023 & -.005 & 6.585 \\
\hline$A$ & 281.6893 & 4.96654 & 4.96692 & -.00038 & -.008 & 6.585 \\
\hline$A$ & 281.6893 & 4.96655 & 4.96692 & -.00037 & -.007 & 6.585 \\
\hline$A$ & $281 \cdot 1393$ & 4.90608 & 4.90636 & -.00028 & -.006 & 6.585 \\
\hline$A$ & 281.4393 & 4.93896 & 4.93929 & -.00033 & -.007 & 6.585 \\
\hline$A$ & 281.4393 & 4.93780 & 4.93929 & -.00149 & -.030 & 5.904 \\
\hline$A$ & 281.4393 & 4.93779 & 4.93929 & -.00150 & -.030 & 5.904 \\
\hline$A$ & 280.6394 & 4.85132 & 4.85200 & -.00068 & -.014 & 5.904 \\
\hline$A$ & 279.4394 & 4.72339 & 4.72402 & -.00063 & -.013 & 5.904 \\
\hline $\mathrm{H}$ & 282.1393 & 5.01753 & 5.01711 & .00042 & .008 & 8.757 \\
\hline $\mathrm{B}$ & 282.0893 & 5.01192 & 5.01150 & .00042 & .008 & 8.757 \\
\hline B & 282.0393 & 5.00628 & 5.00590 & .00038 & .008 & 8.757 \\
\hline $\mathrm{B}$ & $281 \cdot 1500$ & 4.90747 & 4.90753 & -.00006 & -.001 & 7.610 \\
\hline E & 281.4500 & 4.94046 & 4.94047 & -.00001 & -.000 & 7.610 \\
\hline E & 281.7500 & 4.97369 & 4.97365 & .00004 & .001 & 7.610 \\
\hline B & 282.0500 & 5.00718 & 5.00710 & .00008 & .002 & 7.610 \\
\hline B & 282.0500 & 5.00724 & 5.00710 & .00014 & .003 & 7.610 \\
\hline $\mathrm{H}$ & 282.1500 & 5.01841 & 5.01831 & .00010 & .002 & 7.610 \\
\hline$E$ & 282.2500 & 5.02967 & 5.02956 & .00011 & .002 & 7.610 \\
\hline $\mathrm{B}$ & 282.3440 & 5.04045 & 5.04016 & .00029 & .006 & 7.610 \\
\hline $\mathrm{H}$ & 282.3440 & 5.04044 & 5.04016 & .00028 & .005 & 7.610 \\
\hline $\mathrm{E}$ & $281+7500$ & 4.97377 & 4.97365 & .00012 & .002 & 7.610 \\
\hline $\mathrm{E}$ & 281.7500 & 4.97369 & 4.97365 & .00004 & .001 & 7.610 \\
\hline E & 281.6893 & 4.96693 & 4.96692 & .00001 & .000 & 6.516 \\
\hline E & 281.4393 & 4.93935 & 4.93929 & .00006 & .001 & 6.516 \\
\hline $\mathrm{H}$ & 282.1893 & 5.02242 & 5.02236 & .00006 & .001 & 6.516 \\
\hline E & 282.1393 & 5.01690 & 5.01711 & -.00021 & -.004 & 6.516 \\
\hline B & 282.0893 & 5.01135 & 5.01150 & -.00015 & -.003 & 6.516 \\
\hline B & 282.0393 & 5.00573 & 5.00590 & -.00017 & -.003 & 6.516 \\
\hline E & 281.7393 & 4.97239 & 4.97246 & -.00007 & -.001 & 6.516 \\
\hline E & 281.4393 & 4.93925 & 4.93929 & -.00004 & -.001 & 6.516 \\
\hline B & 281.4393 & 4.93873 & 4.93929 & -.00056 & -.011 & 5.899 \\
\hline B & 281.1393 & 4.90599 & 4.90636 & -.00037 & -.007 & 5.899 \\
\hline
\end{tabular}


Table BIII. Comparison of experimental coexisting densities with the present formulation.

\begin{tabular}{|c|c|c|c|c|c|}
\hline \multirow{3}{*}{$\begin{array}{l}\text { EXF } \\
\text { III. }\end{array}$} & \multirow{2}{*}{\multicolumn{2}{|c|}{$\begin{array}{c}\text { TEMFEFiATUFE } \\
K\end{array}$}} & \multicolumn{3}{|c|}{ MENSITY } \\
\hline & & & \multicolumn{2}{|c|}{ MOL/IIM3 } & \multirow[b]{2}{*}{$\%$ IIIFF } \\
\hline & & EXF' & CALC & IIIFF & \\
\hline 1 & 279.150 & 10.674 & 10.692 & -.018 & -.17 \\
\hline 1 & 279.150 & 4.724 & 4.711 & .013 & .28 \\
\hline 1 & 280.150 & 10.290 & 10.290 & .000 & .00 \\
\hline 1 & 280.150 & 5.071 & 5.073 & -.002 & -.05 \\
\hline 1 & 281.150 & 9.767 & 9.762 & .005 & .05 \\
\hline 1 & 281.150 & 5.546 & 5.559 & -.013 & -.24 \\
\hline 1 & 281.650 & 9.387 & 9.390 & -.003 & -.04 \\
\hline 1 & 281.650 & 5.911 & 5.910 & .001 & .02 \\
\hline 1 & 282.150 & 8.780 & 8.770 & .010 & .12 \\
\hline 1 & 282.150 & 6.489 & 6.509 & -.020 & -.30 \\
\hline A & 279.523 & 10.552 & 10.551 & .001 & .01 \\
\hline$A$ & 280.697 & 10.032 & 10.024 & .008 & .08 \\
\hline A & 281.781 & 9.293 & 9.267 & .026 & .28 \\
\hline A & 282.084 & 8.917 & 8.887 & .030 & .34 \\
\hline A & 282.165 & 8.764 & 8.739 & .025 & .29 \\
\hline A & 281.630 & 5.904 & 5.893 & .011 & .18 \\
\hline E & 281.660 & 5.899 & 5.919 & -.020 & -.34 \\
\hline E & 282.165 & 8.757 & 8.739 & .018 & .20 \\
\hline$E$ & 279.317 & 10.627 & 10.630 & -.003 & -.03 \\
\hline
\end{tabular}


Table BIV. Comparison of the experimental speed of sound data with the present formulation.

EXP.II LIENSITY TEMFERATURE MOL/LIM3

\section{K}

\section{GAM}

GAM

GAM

GAM

GAM

GAM

GAM

GAM

GAM

GAM

GAM

GAM

GAM

GAM

GAM

GAM

GAM

GAM

GAM

GAM

GAM

GAM

GAM

GAM

GAM

GAM

GAM

GAM

GAM

GAM

GAM

GAM

GAM

GAM

GAM

GAM

GAM

GAM

GAM

GAM

GAM

GAM

GAM

GAM

GAM

GAM

GAM

GAM

GAM

GAM

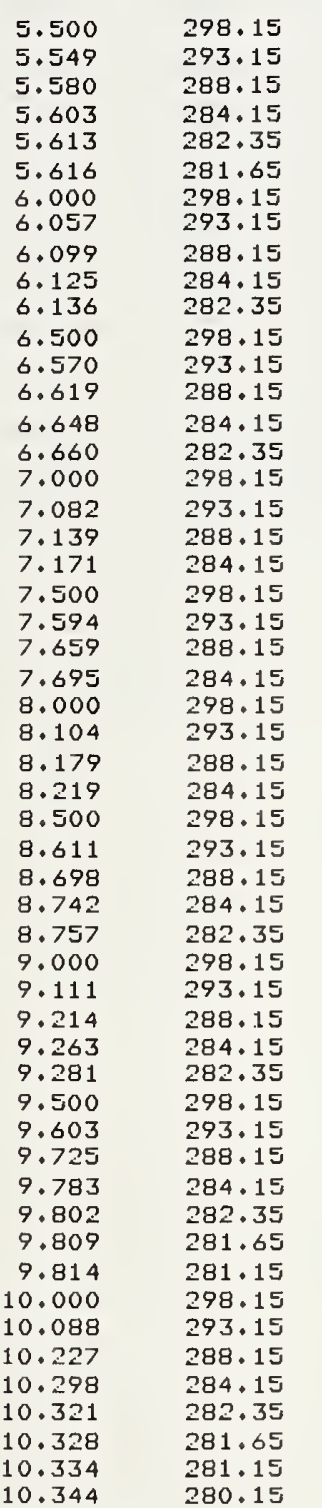

SOUNI UELOCITY

\begin{tabular}{|c|c|c|c|}
\hline & $M$ & & \\
\hline EXP & CALC & IIIFF & 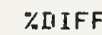 \\
\hline 41.910 & 241.055 & .855 & .35 \\
\hline 32 & 232.661 & .271 & .12 \\
\hline 22.628 & 222.961 & -.333 & -.15 \\
\hline $\begin{array}{l}12.166 \\
05.512\end{array}$ & $\begin{array}{l}212.958 \\
206.612\end{array}$ & $\begin{array}{r}-.792 \\
-1.100\end{array}$ & $\begin{array}{l}-.37 \\
-.54\end{array}$ \\
\hline 01.862 & 203.264 & -1.402 & -.69 \\
\hline $\begin{array}{l}41.573 \\
31.574\end{array}$ & $\begin{array}{l}240.766 \\
231.315\end{array}$ & $\begin{array}{l}.807 \\
.259\end{array}$ & $\begin{array}{l}.3 \\
.1\end{array}$ \\
\hline $\begin{array}{l}19.513 \\
05.844 \\
94.091\end{array}$ & $\begin{array}{l}219.808 \\
206.628 \\
195.808\end{array}$ & $\begin{array}{r}-.295 \\
-.784 \\
-1.717\end{array}$ & $\begin{array}{l}-.13 \\
-.38 \\
-.88\end{array}$ \\
\hline $\begin{array}{l}42.772 \\
31.740 \\
17.774\end{array}$ & $\begin{array}{l}242.006 \\
231.462 \\
217.942\end{array}$ & $\begin{array}{r}.766 \\
.278 \\
-.168\end{array}$ & $\begin{array}{r}.32 \\
.12 \\
-.08\end{array}$ \\
\hline 0.183 & 200.702 & -.519 & -.26 \\
\hline $\begin{array}{l}74.989 \\
45.751\end{array}$ & $\begin{array}{l}179.834 \\
245.075\end{array}$ & $\begin{array}{r}-4.845 \\
.676\end{array}$ & $\begin{array}{r}-2.77 \\
.28\end{array}$ \\
\hline 33.763 & 233.505 & .258 & .11 \\
\hline 17.894 & 217.962 & -.068 & -.03 \\
\hline 96.298 & 196.498 & -.200 & -.1 \\
\hline 50.886 & 250.338 & .548 & .22 \\
\hline 38.059 & 237.944 & .115 & .0 \\
\hline 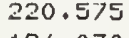 & 220.651 & -.076 & 0 \\
\hline $\begin{array}{l}96.030 \\
58.50 ?\end{array}$ & $\begin{array}{l}195.816 \\
258.204\end{array}$ & & .1 \\
\hline 45.187 & 245.313 & $\begin{array}{r}.120 \\
-.126\end{array}$ & -.0 \\
\hline 26.697 & 226.881 & -.184 & -.08 \\
\hline 00.896 & 200.342 & .554 & .2 \\
\hline 69.043 & 269.075 & -.032 & -.0 \\
\hline 55.621 & 256.097 & -.476 & -.15 \\
\hline 36.918 & 237.450 & -.532 & -.2 \\
\hline 11.682 & 211.236 & .446 & .2 \\
\hline 35.537 & 182.228 & 3.309 & 1.78 \\
\hline 54 & 283.285 & -.431 & -.1 \\
\hline 69.788 & 270.527 & -.739 & -.2 \\
\hline 51.935 & 252.829 & -.894 & -.35 \\
\hline 93 & 228.735 & .058 & $\cdot$ \\
\hline 10.086 & 208.576 & 1.510 & \\
\hline 00.682 & 301.021 & -.339 & -.1 \\
\hline 7.875 & 288.593 & -.718 & -.2 \\
\hline 6 & 272.975 & -1.139 & \\
\hline 51.845 & 252.209 & -.364 & -.1 \\
\hline 38.193 & 237.495 & .698 & .2 \\
\hline 1 . & 229.618 & 1.429 & .6 \\
\hline 224.901 & 222.440 & 2.461 & 1.05 \\
\hline 43 & 322.283 & .060 & .0 \\
\hline 09 & 310.092 & -.274 & -.0 \\
\hline 296.382 & 297.219 & -.837 & -.28 \\
\hline 279.924 & 280.329 & -.405 & -.1 \\
\hline 0 & 269.404 & .257 & . 1 \\
\hline & 264.150 & .788 & .30 \\
\hline 261. & 260.014 & 1.138 & .4 \\
\hline 77 & 249.908 & 2.569 & 1.0 \\
\hline
\end{tabular}


Table BV. Comparison of the isobaric enthalpy differences [21] with the present formulation.

$\begin{array}{lcccccccc}T_{\text {in }} \\ \left({ }^{\circ} \mathrm{C}\right) & \begin{array}{c}T_{\text {out }} \\ \left({ }^{\circ} \mathrm{C}\right)\end{array} & \begin{array}{c}\text { Inlet } \\ \text { Pressure } \\ (\mathrm{MPA})\end{array} & \begin{array}{c}H(\mathrm{exp}) \\ (\mathrm{J} / \mathrm{g})\end{array} & \begin{array}{c}H(\text { eqn }) \\ (\mathrm{J} / \mathrm{g})\end{array} & \begin{array}{c}\Delta H(\exp ) \\ (\mathrm{J} / \mathrm{g})\end{array} & \begin{array}{c}\Delta H(\text { eqn }) \\ (\mathrm{J} / \mathrm{g})\end{array} & \begin{array}{c}\text { Diff } \\ (\mathrm{J} / \mathrm{g})\end{array} & \% \text { Diff } \\ 6.934 & 9.372 & 5.1755 & 25.367 & 25.591 & 6.449 & 6.423 & .026 & 0.4 \\ 6.928 & 9.709 & 5.1749 & 31.816 & 32.014 & 9.741 & 9.433 & .308 & 3.2 \\ 6.931 & 10.041 & 5.1749 & 41.557 & 41.447 & 8.156 & 7.490 & .666 & 8.2 \\ 6.934 & 10.192 & 5.1748 & 49.713 & 48.937 & 8.879 & 8.519 & .360 & 4.0 \\ 6.933 & 10.290 & 5.1748 & 58.592 & 57.456 & 9.599 & 9.659 & -.060 & -0.63 \\ 6.934 & 10.356 & 5.1748 & 68.191 & 67.115 & 13.951 & 13.504 & .447 & 3.2 \\ 6.936 & 10.431 & 5.1747 & 82.142 & 80.619 & 7.461 & 8.141 & -.680 & -9.1 \\ 6.931 & 10.492 & 5.1749 & 89.603 & 88.760 & 3.846 & 4.612 & -.766 & -19.9 \\ 6.932 & 10.537 & 5.1749 & 93.449 & 93.372 & 7.951 & 8.147 & -.196 & -2.5 \\ 6.932 & 10.648 & 5.1749 & 101.400 & 101.519 & 8.268 & 8.448 & -.180 & -2.8 \\ 6.931 & 10.823 & 5.1747 & 109.668 & 109.967 & 8.590 & 8.290 & .300 & 3.55 \\ 6.930 & 11.080 & 5.1747 & 118.258 & 118.257 & & & & \end{array}$


APPENDIX C

Tables of Thermodynamic Properties 
Table CI. The thermodynamic properties of ethylene in the critical region as a function of temperature along isochores $5.75 \mathrm{MOL} / \mathrm{IIM} 3$ ISOCHOFE

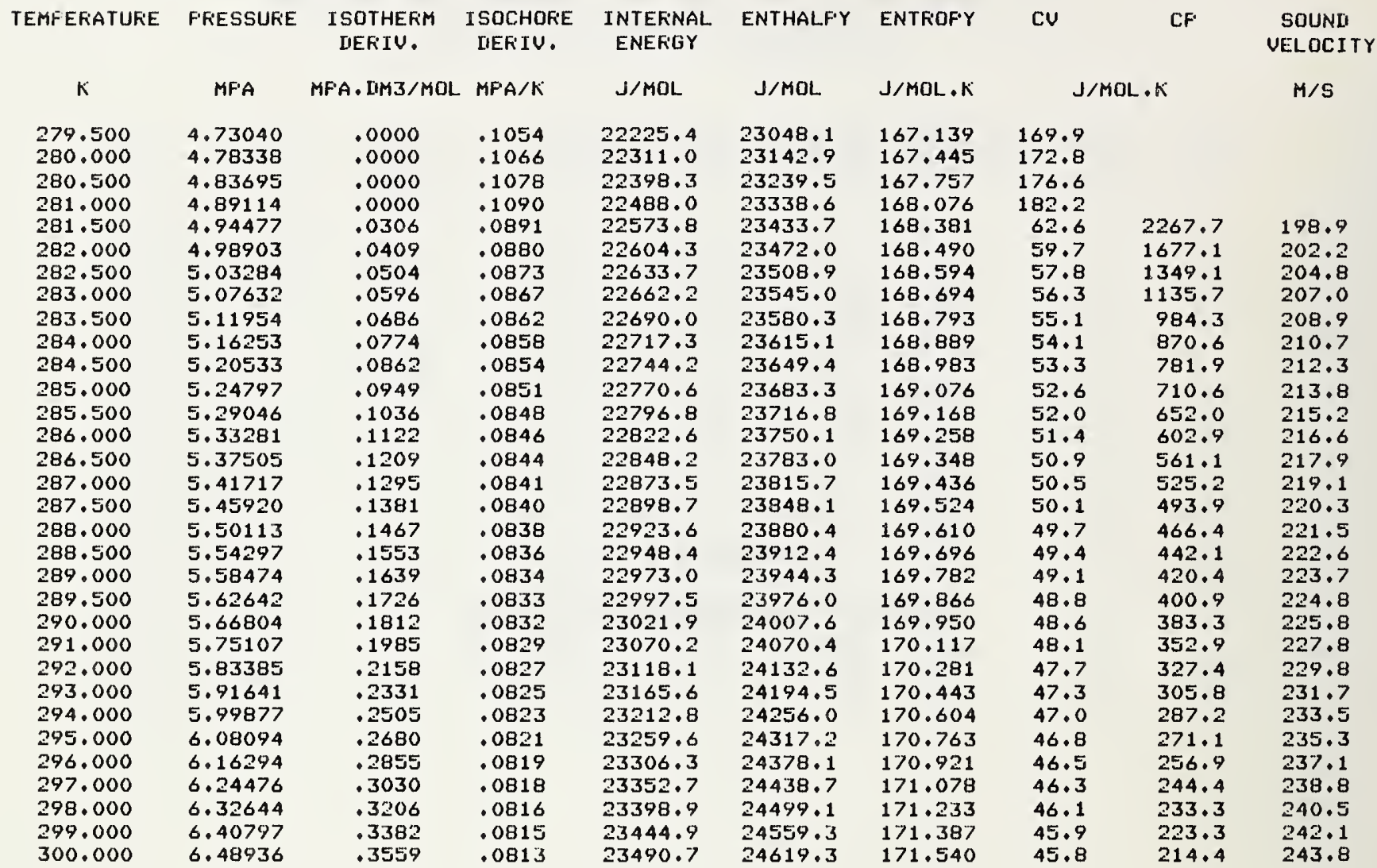


Table CI. (Continued)

6.00 MOL/IM3 ISOCHOFE

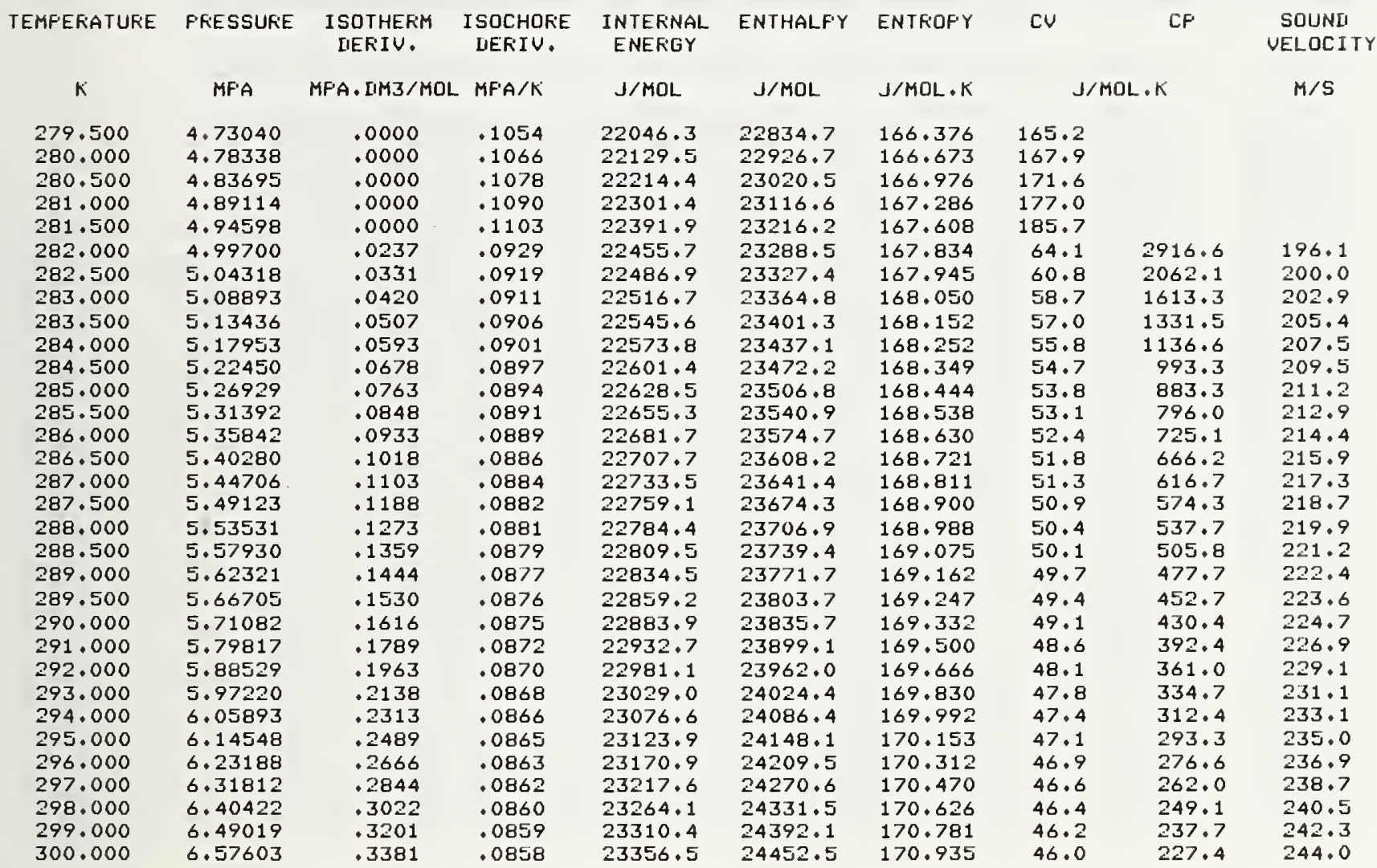


Table CI. (Continued)

\section{$6.25 \mathrm{MOL} / \mathrm{LIM} 3$ ISOCHOFE}

\begin{tabular}{|c|c|c|c|c|c|c|c|c|c|}
\hline TEMPERATURE & PRESSURE & $\begin{array}{l}\text { ISOTHEFM } \\
\text { IIERIV. }\end{array}$ & $\begin{array}{l}\text { ISOCHORE } \\
\text { IIEKIV. }\end{array}$ & $\begin{array}{l}\text { INTEFNAL } \\
\text { ENEFGY }\end{array}$ & ENTHALF'Y & ENTKOFY & cu & $\mathrm{CP}$ & $\begin{array}{l}\text { SOUND } \\
\text { VELOCIT }\end{array}$ \\
\hline$\kappa$ & MF'A & $M F ' A \cdot L M 3 / M O L$ & $M F \cdot A / K$ & $J / M O L$ & J/MOL. & $J / M O L \cdot K$ & \multicolumn{2}{|c|}{$J / M O L \cdot K$} & $M / S$ \\
\hline 279.500 & 4.73040 & .0000 & .1054 & 21881.5 & 22638.3 & 165.673 & 160.8 & & \\
\hline 280.000 & 4.78338 & .0000 & .1066 & 21962.5 & 22727.8 & 165.963 & 163.5 & & \\
\hline 280.500 & 4.83695 & .0000 & .1078 & 22045.1 & 22819.0 & 166.258 & 167.0 & & \\
\hline $\begin{array}{l}281.000 \\
281.500\end{array}$ & $\begin{array}{l}4.89114 \\
4.94598\end{array}$ & $\begin{array}{l}.0000 \\
.0000\end{array}$ & $\begin{array}{l}.1090 \\
.1103\end{array}$ & $\begin{array}{l}22129.8 \\
22217.8\end{array}$ & $\begin{array}{l}22912.4 \\
23009.1\end{array}$ & $\begin{array}{l}166.559 \\
166.872\end{array}$ & $\begin{array}{l}172.2 \\
180.6\end{array}$ & & \\
\hline $\begin{array}{l}282.000 \\
282.500\end{array}$ & $\begin{array}{l}5.00126 \\
5.04976\end{array}$ & $\begin{array}{l}.0111 \\
.0203\end{array}$ & $\begin{array}{l}.0979 \\
.0963\end{array}$ & $\begin{array}{r}22309.7 \\
22343.3\end{array}$ & $\begin{array}{l}23110.0 \\
23151.3\end{array}$ & $\begin{array}{l}167.199 \\
167.317\end{array}$ & $\begin{array}{l}70.7 \\
64.5\end{array}$ & $\begin{array}{l}6299.8 \\
3375.0\end{array}$ & $\begin{array}{l}187.8 \\
194.5\end{array}$ \\
\hline 283.000 & 5.09768 & .0287 & .0954 & 22374.7 & 23190.3 & 167.428 & 61.2 & 2359.0 & 198.6 \\
\hline 283.500 & 5.14523 & .0370 & .0948 & 22404.7 & 23227.9 & 167.534 & 59.0 & 1823.4 & 201.8 \\
\hline 284.000 & 5.19251 & .0452 & .0943 & 22433.8 & 23264.6 & 167.637 & 57.4 & 1489.3 & 204.4 \\
\hline 284.500 & 5.23957 & .0534 & .0939 & 22462.1 & 23300.5 & 167.737 & 56.1 & 1260.2 & 206.7 \\
\hline 285.000 & 5.28645 & .0616 & .0936 & 22489.9 & 23335.7 & 167.834 & 55.0 & 1093.2 & 208.8 \\
\hline 285.500 & 5.33317 & .0698 & .0933 & 22517.2 & 23370.5 & 167.930 & 54.1 & 965.8 & 210.7 \\
\hline 286.000 & 5.37977 & .0781 & .0931 & 22544.0 & 23404.8 & 168.024 & 53.3 & 865.6 & 212.5 \\
\hline 286.500 & 5.42624 & .0864 & .0928 & 22570.5 & 23438.7 & 168.116 & 52.7 & 784.6 & 214.2 \\
\hline 287.000 & 5.47262 & .0947 & .0927 & 22596.7 & $23472 \cdot 3$ & 168.208 & 52.1 & 717.8 & 215.8 \\
\hline 287.500 & 5.51890 & .1031 & .0925 & 22622.6 & 23505.6 & 168.298 & 51.5 & 661.9 & 217.3 \\
\hline 288.000 & 5.56509 & .1116 & .0923 & $22648 \cdot 3$ & 23538.7 & 168.387 & 51.1 & 614.3 & 218.7 \\
\hline 288.500 & 5.61121 & .1200 & .0922 & 22673.7 & 23571.5 & 168.475 & 50.6 & 573.3 & 220.1 \\
\hline 289.000 & 5.65726 & .1285 & .0920 & 22698.9 & 23604.1 & 168.563 & 50.3 & 537.7 & 221.4 \\
\hline 289.500 & 5.70324 & .1371 & .0919 & 22724.0 & 23636.5 & 168.649 & 49.9 & 506.5 & 222.7 \\
\hline 290.000 & 5.74916 & .1457 & .0918 & 22748.8 & 23668.7 & 168.735 & 49.6 & 478.9 & 223.9 \\
\hline 291.000 & 5.84083 & .1630 & .0916 & 22798.1 & 23732.7 & 168.905 & 49.0 & 432.3 & 226.4 \\
\hline 292.000 & 5.93230 & .1804 & .0914 & 22846.9 & 23796.1 & 169.072 & 48.5 & 394.6 & 228.6 \\
\hline 293.000 & 6.02359 & .1979 & .0912 & 22895.2 & 23859.0 & 169.237 & 48.1 & 363.3 & 230.9 \\
\hline 294.000 & 6.11472 & .2156 & .0911 & 22943.1 & 23921.5 & 169.400 & 47.7 & 337.1 & 233.0 \\
\hline 295.000 & 6.20570 & .2334 & .0909 & 22990.7 & 23983.6 & 169.562 & 47.4 & 314.8 & 235.0 \\
\hline 296.000 & 6.29654 & .2513 & .0908 & 23038.0 & 24045.4 & 169.722 & 47.1 & 295.6 & 237.0 \\
\hline 297.000 & 6.38725 & .2693 & .0907 & 23084.9 & 24106.9 & 169.880 & 46.9 & 278.9 & 239.0 \\
\hline 298.000 & 6.47785 & .2874 & .0905 & 23131.7 & 24168.2 & 170.038 & 46.6 & 264.2 & 240.9 \\
\hline 299.000 & 6.56833 & .3056 & .0904 & 23178.3 & 24229.2 & 170.193 & 46.4 & 251.2 & 242.8 \\
\hline 300.000 & 6.65871 & .3239 & .0903 & 23224.6 & 24290.0 & 170.348 & 46.3 & 239.7 & 244.6 \\
\hline
\end{tabular}


Table CI. (Continued)

$6.50 \mathrm{MOL} / \mathrm{DM} 3$ ISOCHOKE

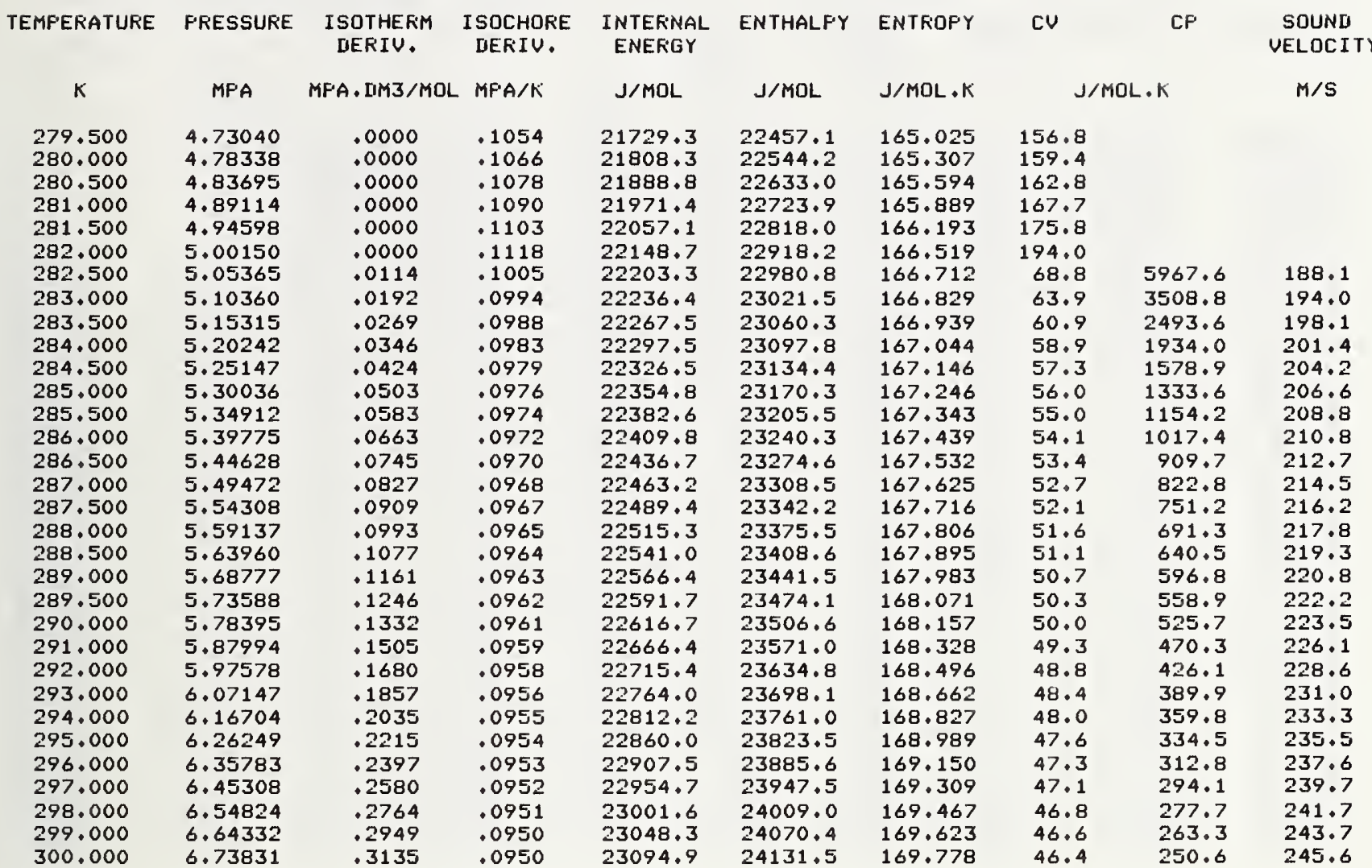


Table CI. (Continued)

6.75 MOL/DM3 ISOCHOFE

\begin{tabular}{|c|c|c|c|c|c|c|c|c|c|}
\hline TEMFERATURE & FRESSUFE & $\begin{array}{l}\text { ISOTHEFM } \\
\text { IIERIV. }\end{array}$ & $\begin{array}{l}\text { ISOCHOFE } \\
\text { IEERIV. }\end{array}$ & $\begin{array}{l}\text { INTEFNAL } \\
\text { ENERGY }\end{array}$ & ENTHALF'Y & ENTROFY & $\mathrm{cV}$ & $\mathrm{CF}$ & $\begin{array}{l}\text { SOUNI } \\
\text { VELOCIT }\end{array}$ \\
\hline$\kappa$ & MFA & MF'A. LIM $3 / M O L$ & $M F^{\prime} A / K$ & $J / M O L$ & $J / M O L$ & $J / M O L \cdot K$ & \multicolumn{2}{|c|}{$J / M O L \cdot K$} & $m / s$ \\
\hline 279.500 & 4.73040 & .0000 & .1054 & 21588.5 & 22289.3 & 164.424 & 153.1 & & \\
\hline 280.000 & 4.78338 & .0000 & .1066 & 21665.6 & 22374.2 & 164.700 & 155.6 & & \\
\hline 280.500 & 4.83695 & .0000 & .1078 & 21744.2 & 22460.8 & 164.980 & 158.9 & & \\
\hline 281.000 & 4.89114 & .0000 & .1090 & 21824.7 & 22549.3 & 165.267 & 163.6 & & \\
\hline 281.500 & 4.94598 & .0000 & .1103 & 21908.3 & 22641.0 & 165.565 & 171.4 & & \\
\hline 282.000 & 5.00150 & .0000 & .1118 & 21997.6 & 22738.6 & 165.882 & 188.9 & & \\
\hline 282,500 & 5.05576 & .0060 & .1042 & 22067.4 & 22816.4 & 166.129 & 73.8 & 11381.1 & 180.9 \\
\hline 283.000 & 5.10756 & .0129 & .1031 & 22102.1 & 22858.8 & 166.252 & 66.4 & 5185.4 & 189.5 \\
\hline 283.500 & 5.15895 & .0200 & .1025 & 22134.3 & 22898.6 & 166.365 & 62.6 & 3337.5 & 194.7 \\
\hline 284,000 & 5.21009 & .0272 & .1021 & 22165.0 & 22936.9 & 166.473 & 60.1 & 2447.9 & 198.7 \\
\hline 284.500 & 5.26105 & .0346 & .1018 & 22194.6 & 22974.0 & 166.578 & 58.3 & 1926.5 & 201.9 \\
\hline 285.000 & 5.31187 & .0422 & .1015 & 22223.4 & 23010.3 & 166.679 & 56.9 & 1585.0 & 204.7 \\
\hline 285.500 & 5.36258 & .0499 & .1013 & 22251.5 & 23045.9 & 166.777 & 55.7 & 1344.8 & 207.3 \\
\hline 286.000 & 5.41320 & .0577 & .1012 & 22279.1 & 23081.0 & 166.874 & 54.7 & 1167.0 & 209.5 \\
\hline 286.500 & 5.46374 & .0657 & .1010 & 22306.2 & 23115.7 & 166.969 & 53.9 & 1030.3 & 211.7 \\
\hline 287,000 & 5.51421 & .0738 & .1009 & 22333.0 & 23149.9 & 167.062 & 53.1 & 922.1 & 213.6 \\
\hline 287.500 & 5.56463 & .0820 & 1008 & 22359.4 & 23183.8 & 167.154 & 52.5 & 834.5 & 215.5 \\
\hline 288,000 & 5.61499 & .0902 & .1007 & 22385.5 & 23217.3 & 167.244 & 51.9 & 762.1 & 217.2 \\
\hline $288 \cdot 500$ & 5.66531 & .0986 & .1006 & 22411.3 & 23250.6 & 167.334 & 51.4 & 701.5 & 218.9 \\
\hline 289.000 & 5.71559 & .1070 & .1005 & 22436.9 & 23283.7 & 167.423 & 51.0 & 649.9 & 220.5 \\
\hline 289.500 & 5.76583 & .1155 & .1005 & 22462.3 & 23316.5 & 167.511 & 50.6 & 605.5 & 222.0 \\
\hline $\begin{array}{l}290.000 \\
291.000\end{array}$ & $\begin{array}{l}5.81604 \\
5.91637\end{array}$ & $\begin{array}{r}.1241 \\
.1415\end{array}$ & $\begin{array}{r}.1004 \\
.1003\end{array}$ & $\begin{array}{l}22487.5 \\
22537.4\end{array}$ & $\begin{array}{l}23349.1 \\
23413.9\end{array}$ & $\begin{array}{l}167.598 \\
167.769\end{array}$ & $\begin{array}{l}50.2 \\
49.6\end{array}$ & $\begin{array}{l}566.9 \\
503.4\end{array}$ & $\begin{array}{l}223.5 \\
226.4\end{array}$ \\
\hline 292.000 & 6.01660 & .1592 & .1002 & 22586.7 & 23478.0 & 167.938 & 49.0 & 453.1 & 229.0 \\
\hline 293.000 & 6.11673 & .1770 & .1001 & 22635.4 & 23541.6 & 168,105 & 48.5 & 412.5 & 231.6 \\
\hline 294.000 & 6.21679 & .1951 & .1000 & 22683.8 & 23604.8 & 168.270 & 48.1 & 379.0 & 234.0 \\
\hline 295.000 & 6.31677 & .2134 & .1000 & 22731.7 & 23667.5 & 168.432 & 47.8 & 350.9 & 236.4 \\
\hline 296.000 & 6.41669 & .2318 & .0999 & 22779.3 & 23730.0 & 168.594 & 47.5 & 327.1 & 238.6 \\
\hline 297.000 & 6.51655 & .2504 & .0998 & 22826.7 & 23792.1 & 168.753 & 47.2 & 306.6 & 240.8 \\
\hline 298.000 & 6.61636 & .2692 & .0998 & 22873.7 & 23853.9 & 168.911 & 46.9 & 288.8 & 243.0 \\
\hline 299.000 & 6.71611 & .2881 & .0997 & 22920.6 & 23915.5 & 169.068 & 46.7 & 273.2 & $245+1$ \\
\hline 300.000 & 6.81582 & .3072 & .0997 & 22967.2 & 23976.9 & 169.224 & 46.5 & 259.5 & 247.1 \\
\hline
\end{tabular}


Table CI. (Continued)

$7.00 \mathrm{MOL} / \mathrm{MM} 3$ ISOCHORE

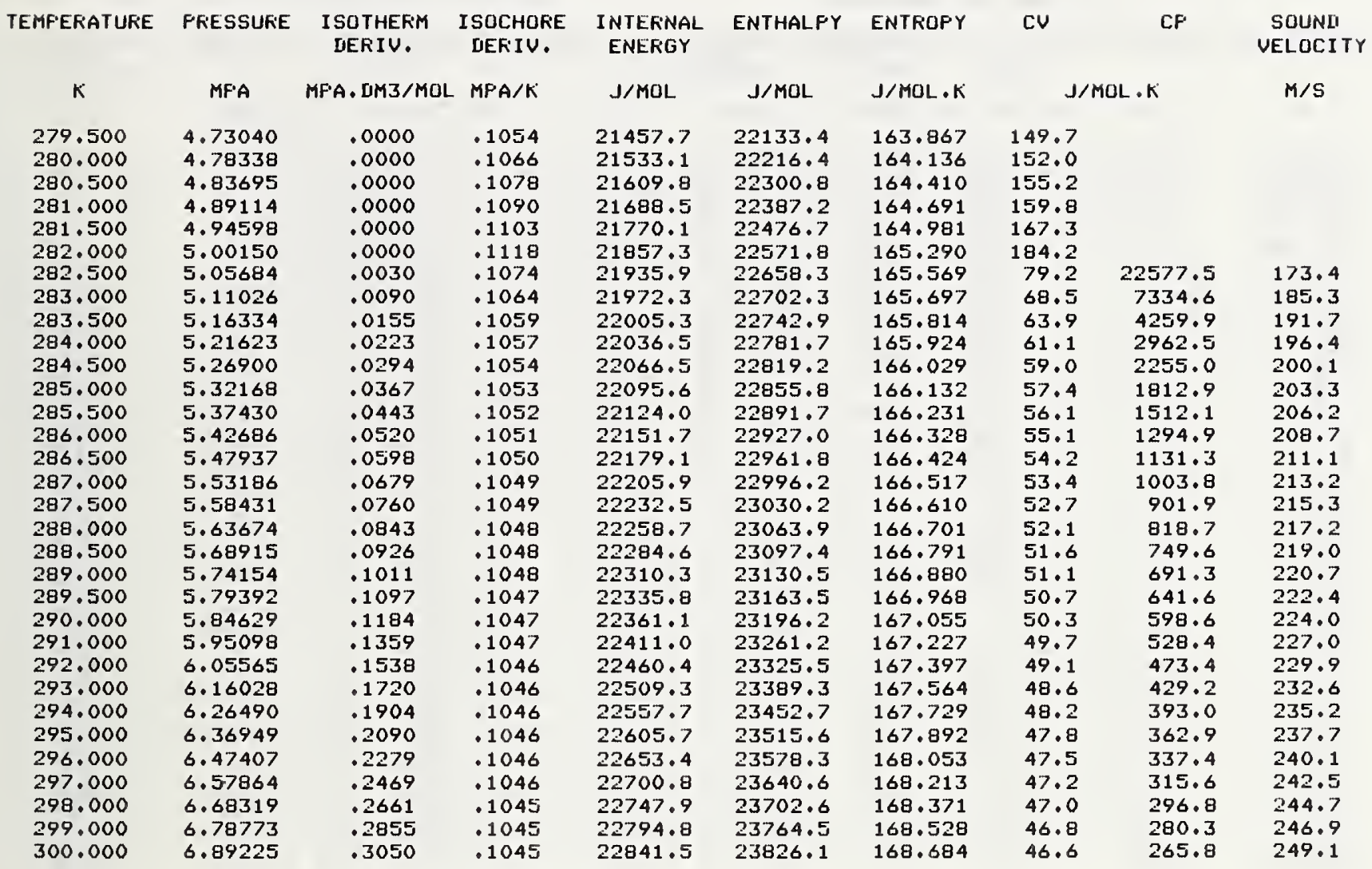


Tabie CI. (Continued)

7.25 MOL/DM3 ISOCHORE

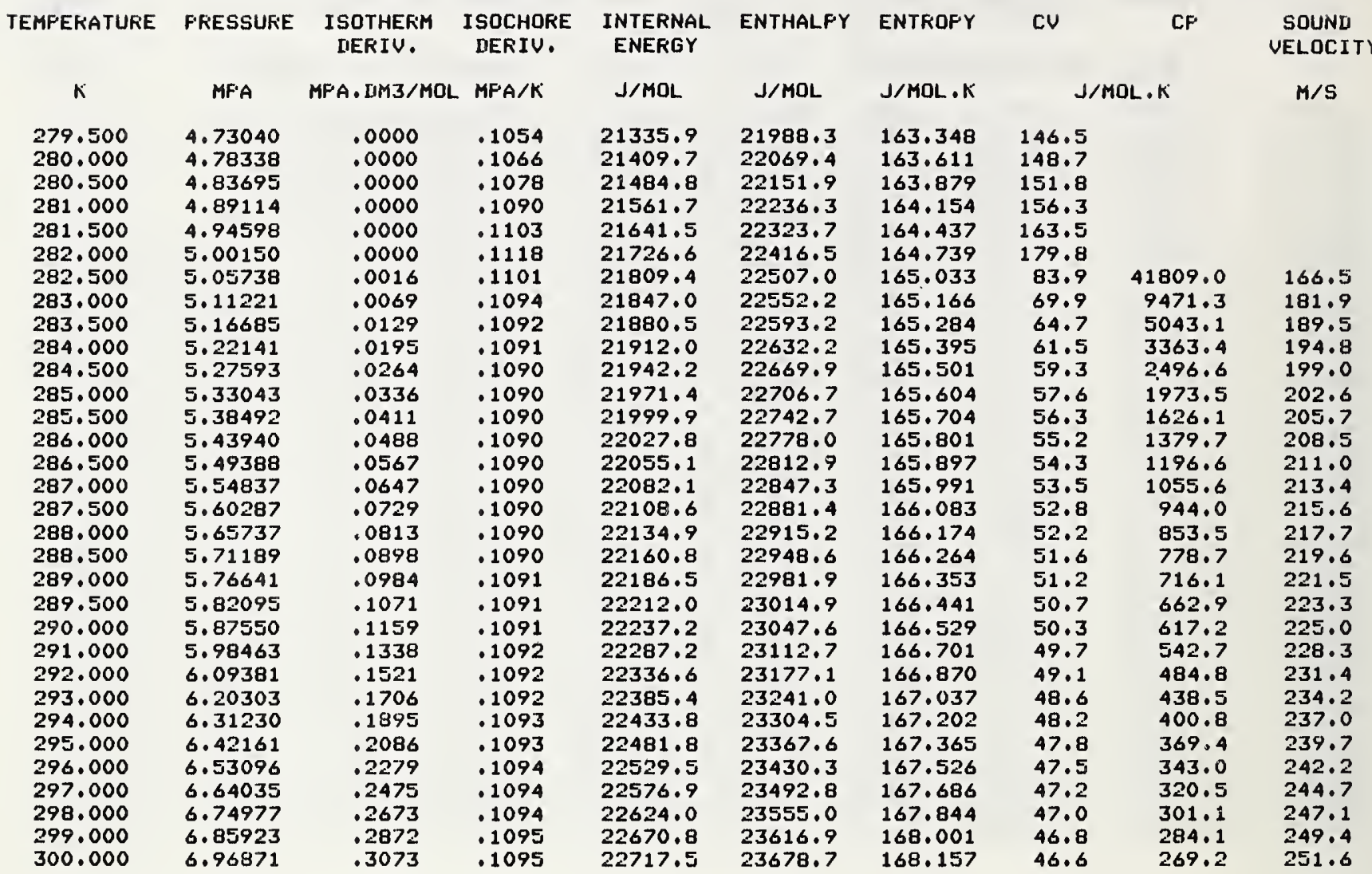


Table CI. (Continued)

$7.50 \mathrm{MOL} / \mathrm{MM} 3$ ISOCHORE

TEMPERATURE FRESSURE ISOTHERM ISOCHORE INTERNAL ENTHALPY ENTROFY DERIV. IIERIV. ENERGY

$\kappa$ MF'A

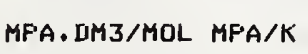

$\begin{array}{lll}4.73040 & .0000 & .1054 \\ 4.78338 & .0000 & .1066 \\ 4.83695 & .0000 & .1078 \\ 4.89114 & .0000 & .1090 \\ 4.94598 & .0000 & .1103 \\ 5.00150 & .0000 & .1118 \\ 5.05769 & .0011 & .1122 \\ 5.11379 & .0060 & .1122 \\ 5.16992 & .0119 & .1123 \\ 5.22611 & .0184 & .1124 \\ 5.28236 & .0254 & .1126 \\ 5.33867 & .0326 & .1127 \\ 5.39503 & .0402 & .1128 \\ 5.45145 & .0480 & .1129 \\ 5.50792 & .0560 & .1130 \\ 5.56444 & .0643 & .1131 \\ 5.62101 & .0727 & .1132 \\ 5.67762 & .0812 & .1133 \\ 5.73428 & .0899 & .1134 \\ 5.79098 & .0987 & .1134 \\ 5.84772 & .1077 & .1135 \\ 5.90450 & .1167 & .1136 \\ 6.01817 & .1351 & .1137 \\ 6.13198 & .1540 & .1139 \\ 6.24592 & .1731 & .1140 \\ 6.35998 & .1925 & .1141 \\ 6.47414 & .2123 & .1142 \\ 6.58839 & .2322 & .1143 \\ 6.70275 & .2524 & .1144 \\ 6.81719 & .2729 & .1145 \\ 6.93170 & .2935 & .1146 \\ 7.04630 & .3143 & .1146\end{array}$

$J / M O L$

21222.2 21294.5 21368.0 21443.4 21521.4 21604.6 21688.3 21726.4 21760.0 21791.5 21821.6 21850.8 21879.2 21907.0 21934.3 21961.1 21987.6 22013.8 22039.7 22065.3 22090.7 22115.9 22165.8 22215.0 22263.7 22312.0 22359.9 22407.5 22454.8 22501.8 22548.6 22595.2

$\begin{array}{ll}J / M O L & J / M O L \cdot K \\ 21852.9 & 162.863 \\ 21932.3 & 163.122 \\ 22012.9 & 163.384 \\ 22095.5 & 163.652 \\ 22180.9 & 163.930 \\ 22271.5 & 164.225 \\ 22362.6 & 164.522 \\ 22408.2 & 164.656 \\ 22449.3 & 164.775 \\ 22488.3 & 164.886 \\ 22525.9 & 164.992 \\ 22562.6 & 165.094 \\ 22598.5 & 165.194 \\ 22633.8 & 165.291 \\ 22668.7 & 165.387 \\ 22703.0 & 165.480 \\ 22737.1 & 165.572 \\ 22770.8 & 165.663 \\ 22804.2 & 165.753 \\ 22837.4 & 165.842 \\ 22870.4 & 165.930 \\ 22903.2 & 166.017 \\ 22968.2 & 166.188 \\ 23032.6 & 166.357 \\ 23096.5 & 166.524 \\ 23160.0 & 166.689 \\ 23223.2 & 166.851 \\ 23286.0 & 167.012 \\ 23348.5 & 167.172 \\ 23410.8 & 167.330 \\ 23472.8 & 167.486 \\ 23534.7 & 167.642\end{array}$

cy CF SOUND
YELOCITY

$\mathrm{J} / \mathrm{MOL} \cdot \mathrm{K}$

$\mathrm{M} / \mathrm{S}$

143.5

145.7

148.7

153.0

160.0

175.7

86.2

70.3

64.7

61.5

59.2

57.5

56.2

55.1

54.1

53.3

52.6

52.0

51.5

51.0

50.6

50.2

49.5

49.0

48.5

48,1

47.7

47.4

47.2

46.9

46.7

46.5

$\begin{array}{rl}60195.4 & 161.9 \\ 10666.2 & 179.9 \\ 5408.3 & 188.2 \\ 3528.7 & 194.1 \\ 2586.8 & 198.7 \\ 2028.4 & 202.6 \\ 1661.9 & 205.9 \\ 1404.4 & 209.0 \\ 1214.3 & 211.7 \\ 1068.7 & 214.3 \\ 953.8 & 216.6 \\ 861.0 & 218.9 \\ 784.7 & 221.0 \\ 720.8 & 223.0 \\ 666.7 & 224.9 \\ 620.2 & 226.7 \\ 544.8 & 230.2 \\ 486.2 & 233.4 \\ 439.6 & 236.5 \\ 401.5 & 239.4 \\ 370.0 & 242.2 \\ 343.5 & 244.9 \\ 320.9 & 247.5 \\ 301.4 & 250.0 \\ 284.4 & 252.4 \\ 269.5 & 254.8\end{array}$


Table CI. (Continued)

$7.75 \mathrm{MOL} / \mathrm{LM} 3$ ISOCHOKE

TEMFERATURE PFESSURE ISOTHERM ISOCHORE INTERNAL ENTHALFY ENTFOFY
IIERIV.
IIERIV.

\author{
J/MOL
}

21115.9
21186.7

21186.7
21258.8

21332.6

21409.1

21490.6

21572.5

21610.1

21643.3

21674.6

21704.4

$21733+4$

21761.6

21789.1

21816.2

21842.9

21869.2

21895.3

21921.0

21946.5

21971.7

21996.8

22046.4

22095.5

22144.0

22192.1

22239.8

22287.2

22334.4

$22381+3$

22427.9

22474.4
J/MOL

21726.3

21803.9

21882.9

21963.7

22047.3

22135.9

22225.1

22270.1

22310.8

22349.5

22386.9

$22423+3$

22459.0

22494.1

22563.0

22596.9

22630.5

22663.8

22696.9

22729.8

22762.5

22827.4

22891.7

22955.6

23019.0

23145.0

23207.5

23269.9

23332.0

23393.9
$22528 \cdot 8$

J/MOL.K

162.410

162.663

162.921

163.183

163.744

164.035

164.168

164.285

164.395

164.500

164.602

164.701

164.797

164.892

164.985

165.077

165.167

165.256

165.344

165.432

165.518

165.689

165.857

166.023

166.187

166.349

166.510

166.669

166.826

166.983
23082.2

163.455

$.3264 \quad .1200$
cV

CF

J/MOL.K

140.7

142.8

145.7

149.9

156.7

171.9

85.0

69.5

64.1

60.9

58.7

57.1

55.7

54.7

53.8

53.0

52.3

51.7

51.2

50.7

50.3

50.0

49.3

48.8

48.3

47.9

47.6

47.3

47.0

46.8

46.6

46.4
57363.7

10192.5

5189.0

3396.6

2496.5

1961.9

1610.6

1363.3

1180.6

1040.4

929.7

840.2

766.5

704.8

652.4

607.5

534.4

477.6

432.3

395.3

364.7

338.8

316.8

297.7

281.2

266.6
SOUNI

VELOCITY

$m / s$

160.5

179.4

188.3

194.4

199.3

203.5

207.1

210.3

213.2

215.9

218.4

220.8

223.0

225.1

227.1

229.1

232.7

236.2

239.4

242.5

245.4

248.2

250.9

253.5

256.1

258.6 
Table CI. (Continued)

$8.00 \mathrm{MOL} /$ IIM3 ISOCHOKE

\begin{tabular}{|c|c|c|c|c|c|c|c|c|c|}
\hline TEMFEF'ATUF'E & FFESSURE & $\begin{array}{l}\text { I SOTHERM } \\
\text { LIEEIV. }\end{array}$ & $\begin{array}{l}\text { ISOCHORE } \\
\text { LIEKIIV. }\end{array}$ & $\begin{array}{l}\text { INTEKNAL } \\
\text { ENEF'GY }\end{array}$ & ENTHALFYY & ENTKOFY & cU & $\mathrm{CF}$ & $\begin{array}{l}\text { SOUNI } \\
\text { VELOCITY }\end{array}$ \\
\hline K & MFA & $M F^{\prime} A \cdot I I M 3 / M O L$ & $M F A / K$ & $\mathrm{~J} / \mathrm{MOL}$ & $\mathrm{J} / \mathrm{MOL}$ & $J / M O L \cdot K$ & \multicolumn{2}{|c|}{$J / M O L \cdot K$} & $M / S$ \\
\hline 280.000 & 4.78338 & .0000 & .1066 & 21085.7 & 21683.6 & 162.234 & 140.1 & & \\
\hline 280.500 & 4.83695 & .0000 & .1078 & 21156.4 & 21761.1 & 162.486 & 142.9 & & \\
\hline 281.000 & 4.89114 & .0000 & .1090 & 21228.8 & 21840.2 & 162.744 & 147.0 & & \\
\hline $\begin{array}{l}281.500 \\
282.000\end{array}$ & $\begin{array}{l}4.94598 \\
5.00150\end{array}$ & $\begin{array}{l}.0000 \\
.0000\end{array}$ & $\begin{array}{l}.1103 \\
.1118\end{array}$ & $\begin{array}{l}21303.8 \\
21383.6\end{array}$ & $\begin{array}{l}21922.0 \\
22008.8\end{array}$ & $\begin{array}{l}163.010 \\
163.294\end{array}$ & $\begin{array}{l}153.5 \\
168.3\end{array}$ & & \\
\hline $\begin{array}{l}283.000 \\
383.500\end{array}$ & 5.11696 & $\begin{array}{l}.0075 \\
.0142\end{array}$ & $\begin{array}{r}1180 \\
.1188\end{array}$ & $\begin{array}{r}21497.8 \\
21530.3\end{array}$ & $\begin{array}{l}22137.4 \\
22177.3\end{array}$ & $\begin{array}{l}163.698 \\
163.813\end{array}$ & 67.7 & $\begin{array}{l}8299.2 \\
4470.0\end{array}$ & $\begin{array}{l}180.8 \\
189.7\end{array}$ \\
\hline $\begin{array}{l}284.000 \\
284.500\end{array}$ & $\begin{array}{l}5.23578 \\
5.29564\end{array}$ & $\begin{array}{l}.0215 \\
.0292\end{array}$ & $\begin{array}{l}.1195 \\
.1200\end{array}$ & $\begin{array}{l}21561.0 \\
21590.4\end{array}$ & $\begin{array}{l}22215.4 \\
22252.3\end{array}$ & $\begin{array}{l}163.921 \\
164.024\end{array}$ & $\begin{array}{l}59.9 \\
57.9\end{array}$ & $\begin{array}{l}3008.7 \\
2250.3\end{array}$ & $\begin{array}{l}196.1 \\
201.2\end{array}$ \\
\hline 285.000 & 5.35575 & .0372 & .1204 & 21618.9 & 22288.4 & 164.124 & 56.3 & 1790.3 & 205.5 \\
\hline $\begin{array}{l}285.500 \\
286.000\end{array}$ & $\begin{array}{l}5.41605 \\
5.47654\end{array}$ & $\begin{array}{l}.0456 \\
.0542\end{array}$ & $\begin{array}{r}.1208 \\
.1211\end{array}$ & $\begin{array}{l}21646.7 \\
21674.0\end{array}$ & $\begin{array}{l}22323.7 \\
22358.5\end{array}$ & $\begin{array}{l}164.222 \\
164.317\end{array}$ & $\begin{array}{l}55.1 \\
54.0\end{array}$ & $\begin{array}{l}1483.3 \\
1264.7\end{array}$ & $\begin{array}{l}209.2 \\
212.6\end{array}$ \\
\hline 289.000 & 5.84247 & .1096 & .1227 & 21829.8 & 22560.1 & 164.859 & 50.3 & 670.4 & 228.1 \\
\hline 289.500 & 5.90386 & .1194 & .1229 & 21854.9 & 22592.9 & 164.946 & 49.9 & 622.2 & 230.2 \\
\hline 290,000 & 5.96536 & .1293 & .1231 & 21879.8 & 22625.4 & 165.032 & 49.6 & 580.6 & 232.3 \\
\hline 291.000 & 6.08862 & .1493 & .1234 & 21929.0 & 22690.1 & 165.202 & 49.0 & 512.9 & 236.1 \\
\hline 292.000 & 6.21221 & .1698 & .1237 & 21977.7 & 22754.3 & 165.369 & 48.5 & 459.9 & 239.7 \\
\hline 293.000 & 6.33610 & .1906 & .1240 & 22026.0 & 22818.0 & 165.534 & 48.0 & 417.5 & 243.1 \\
\hline 294,000 & 6.46027 & .2118 & .1243 & 22073.8 & 22881.3 & 165.697 & 47.7 & 382.8 & 246.3 \\
\hline 295.000 & 6.58470 & .2332 & .1245 & 22121.3 & 22944.4 & 165.858 & 47.3 & 353.9 & 249.3 \\
\hline 296.000 & 6.70935 & .2549 & .1248 & 22168.5 & 23007.2 & 166.018 & 47.1 & 329.5 & 252.2 \\
\hline 297.000 & 6.83422 & .2768 & .1250 & 22215.4 & 23069.7 & 166.176 & 46.8 & 308.6 & 255.1 \\
\hline 298.000 & 6.95928 & .2990 & .1252 & 22262.1 & 23132.0 & 166.333 & 46.6 & 290.5 & 257.8 \\
\hline 299.000 & 7.08453 & .3214 & .1253 & 22308.6 & 23194.2 & 166.489 & 46.4 & 274.8 & 260.4 \\
\hline 300.000 & 7.20995 & .3439 & .1255 & 22354.9 & 23256.2 & 166.643 & 46.2 & 260.9 & 263.0 \\
\hline
\end{tabular}


Table CI. (Continued)

8.25 MOL/LM3 ISOCHOKE

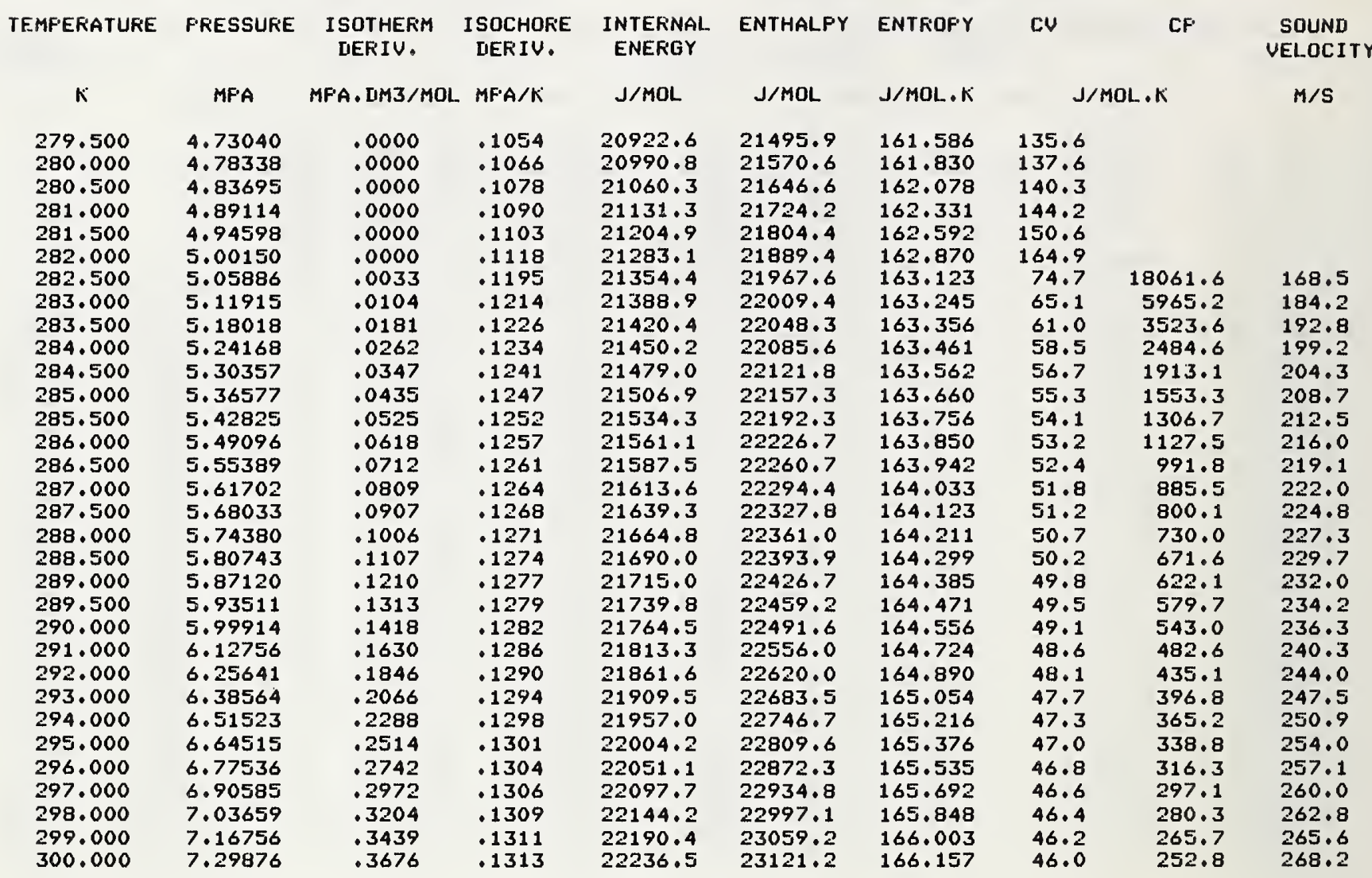


Table CI. (Continued)

8.50 MOL/LM3 ISOCHORE

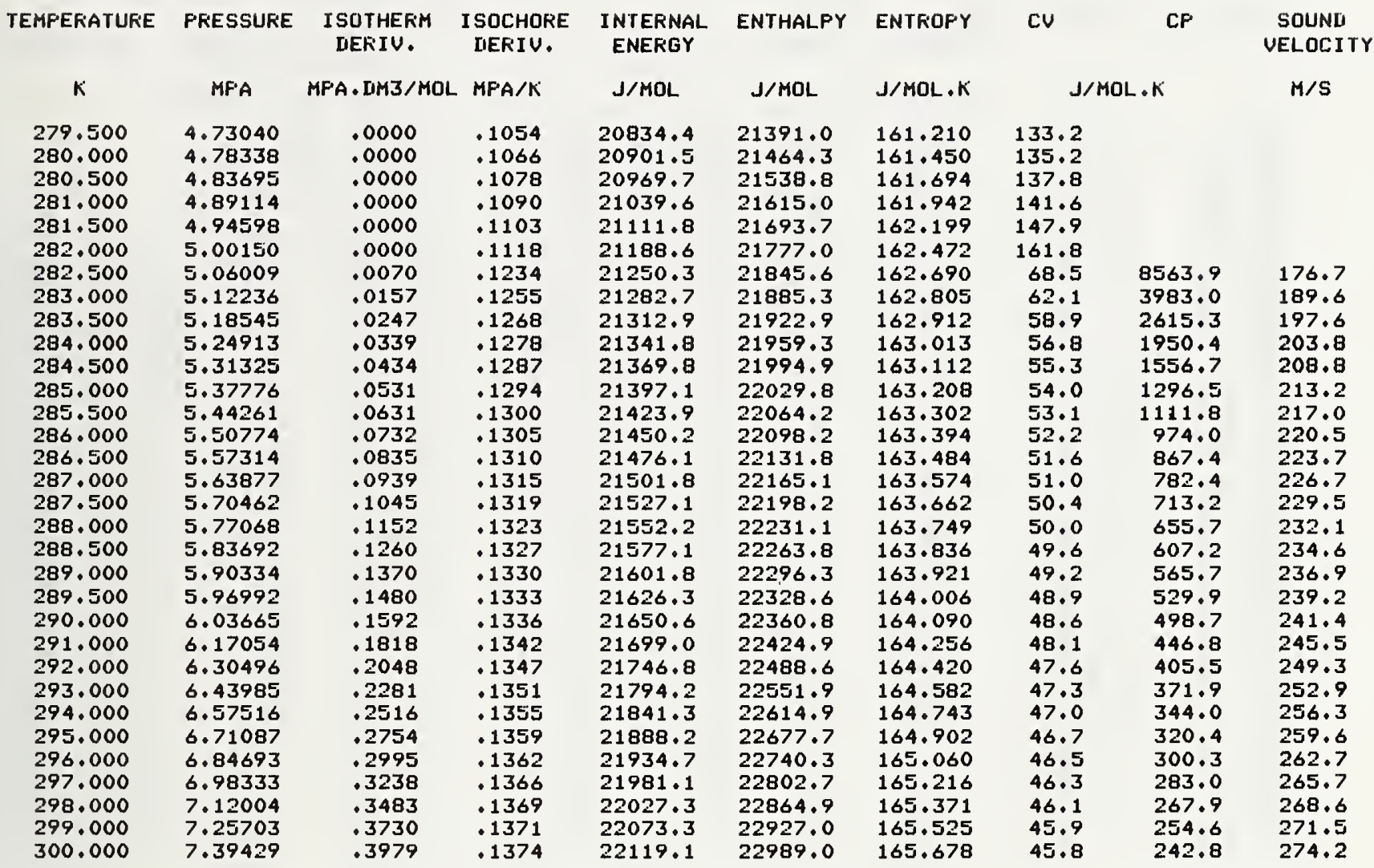


Table CI. (Continued)

8.75 MOL/LM3 ISOCHORE

TEIFEFATUKE PRESSURE ISOTHERM ISOCHOFE INTEKNAL ENTHALFY ENTROFY
DERIV.

K

279.500

280.000

280.500

281.000

281.500

282.000

282.500

283.000

283.500

284.000

284.500

285.000

285.500

286.000

286.500

287.000

287.500

288.000

288.500

289.000

289.500

290.000

291.000

292.000

293.000

294.000

295.000

296.000

297.000

298.000

299.000

300.000

$\begin{array}{ccc}\text { MFA } & \text { MFA. DM3/MOL } & \text { MFA/K } \\ 4.73040 & .0000 & .1054 \\ 4.78338 & .0000 & .1066 \\ 4.83695 & .0000 & .1078 \\ 4.89114 & .0000 & .1090 \\ 4.94598 & .0000 & .1103 \\ 5.00150 & .0000 & .1118 \\ 5.06266 & .0142 & .1282 \\ 5.12732 & .0247 & .1303 \\ 5.19284 & .0351 & .1317 \\ 5.25898 & .0457 & .1328 \\ 5.32563 & .0564 & .1337 \\ 5.39271 & .0672 & .1345 \\ 5.46016 & .0782 & .1353 \\ 5.52795 & .0893 & .1359 \\ 5.59603 & .1006 & .1365 \\ 5.66439 & .1120 & .1370 \\ 5.73300 & .1235 & .1375 \\ 5.80184 & .1351 & .1379 \\ 5.87090 & .1468 & .1383 \\ 5.94016 & .1586 & .1387 \\ 6.00960 & .1704 & .1391 \\ 6.07922 & .1824 & .1394 \\ 6.21896 & .2066 & .1401 \\ 6.35931 & .2311 & .1406 \\ 6.50020 & .2559 & .1411 \\ 6.64158 & .2809 & .1416 \\ 6.78342 & .3062 & .1421 \\ 6.92568 & .3317 & .1425 \\ 7.06832 & .3574 & .1428 \\ 7.21132 & .3832 & .1432 \\ 7.35466 & .4093 & .1435 \\ 7.49830 & .4355 & .1438 \\ & & \end{array}$

J/MOL

20751.3

20817.3

20894.4

20953.0

21024.0

21099.4

21147.9

21178.3

21207.2

21235.1

21262.3

21288.8

21315.0

21340.7

21366.2

21391.3

21416.2

21440.9

21465.4

21489.8

21513.9

21538.0

21585.7

21633.0

21680.0

21726.7

21773.1

21819.3

21865.3

21911.2

21956.9

22002.4

$21726.5 \quad 162.268$

$21939.0 \quad 162.856$
J/MOL

J/MOL.K

21292.0

160.856

cu

$\mathrm{CF}$

SOUNL

UELOCITY

$21364.0 \quad 161.092$

$21437.2 \quad 161.331$

$21512.0 \quad 161.576$

$21589.3 \quad 161.828$

$21671.0 \quad 162.096$

$21764.3 \quad 162.375$

$21800.7 \quad 162.477$

$21836.1 \quad 162.576$

$21870.9 \quad 162.671$

$21905.2 \quad 162.764$

$21972.5 \quad 162.946$

$22005.7 \quad 163.035$

$22038.7 \quad 163.123$

$22071.4 \quad 163.209$

$22104.0 \quad 163.295$

$22136.4 \quad 163.380$

$22168.6 \quad 163.465$

$22200.8 \quad 163.548$

$22232.8 \quad 163.631$

$22296.5 \quad 163.795$

$22359.8 \quad 163.958$

$22422.9 \quad 164.118$

$22485.7 \quad 164.277$

$22549.3 \quad 164.435$

$22610.8 \quad 164.591$

$22673.1 \quad 164.747$

$22735.3 \quad 164.901$

$22797.4 \quad 165.054$

22859.4165 .206
J/MOL.K

$M / S$

131.0

133.0

135.5

139.2

145.3

158.8

63.1

59.0

56.6

55.0

53.7

52.7

51.9

51.2

50.6

50.1

49.6

49.2

48.8

48.5

48.2

48.0

47.5

47.1

46.8

46.5

46.3

46.1

45.9

45.8

45.6

45.5

$\begin{array}{rl}4324.0 & 186.5 \\ 2598.0 & 196.9 \\ 1884.2 & 204.1 \\ 1487.1 & 209.9 \\ 1232.5 & 214.8 \\ 1055.0 & 219.1 \\ 924.0 & 222.9 \\ 823.2 & 226.4 \\ 743.2 & 229.6 \\ 678.2 & 232.6 \\ 624.3 & 235.4 \\ 578.9 & 238.0 \\ 540.1 & 240.5 \\ 506.6 & 242.9 \\ 477.3 & 245.2 \\ 451.6 & 247.4 \\ 408.3 & 251.6 \\ 373.5 & 255.5 \\ 344.7 & 259.2 \\ 320.7 & 262.7 \\ 300.2 & 266.0 \\ 282.6 & 269.2 \\ 267.4 & 272.3 \\ 253.9 & 275.3 \\ 242.1 & 278.2 \\ 231.5 & 281.0\end{array}$


Table CI. (Continued)

$9.00 \mathrm{MOL} / \mathrm{IM} 3$ ISOCHOFE

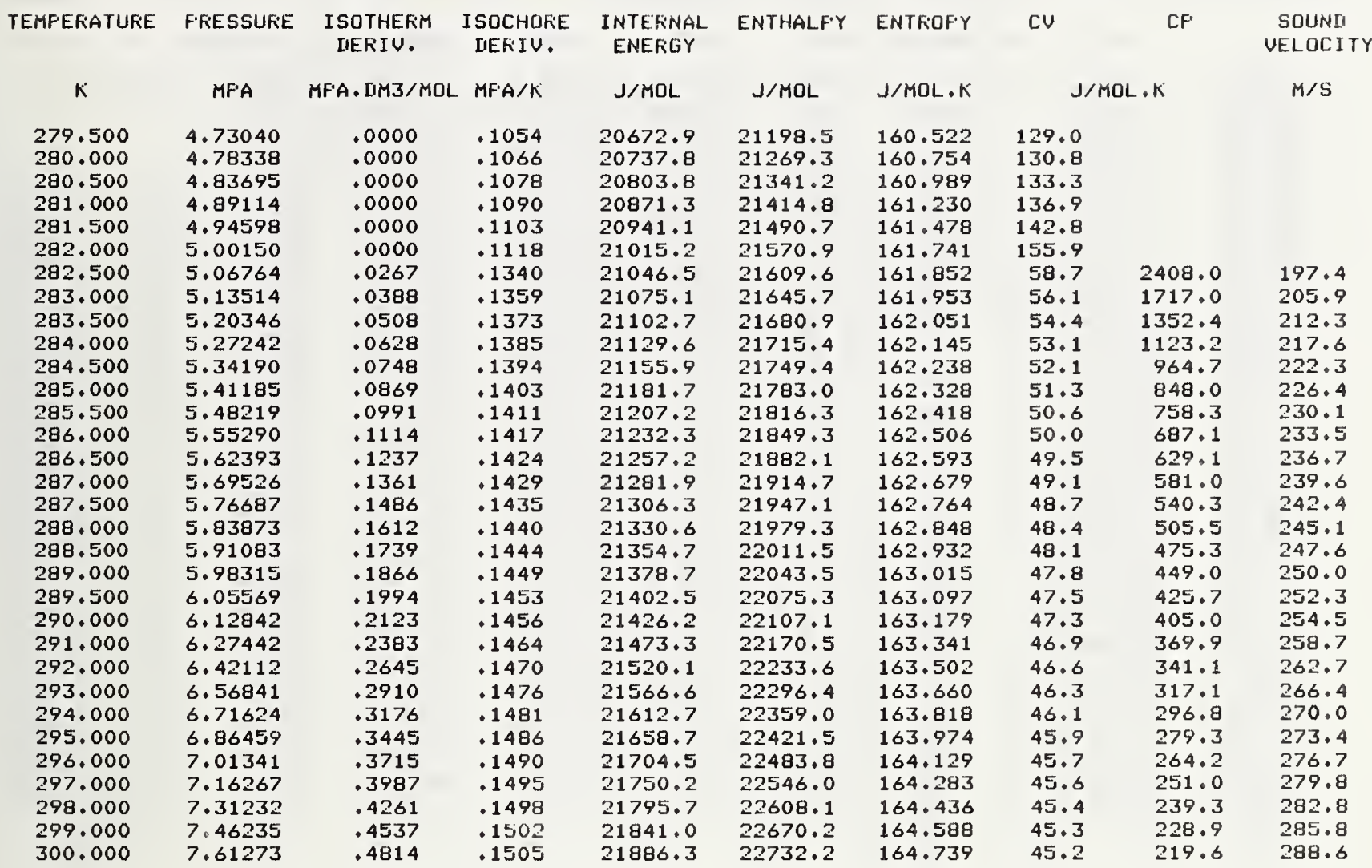


9.25 MOL/LM3 ISOCHOKE

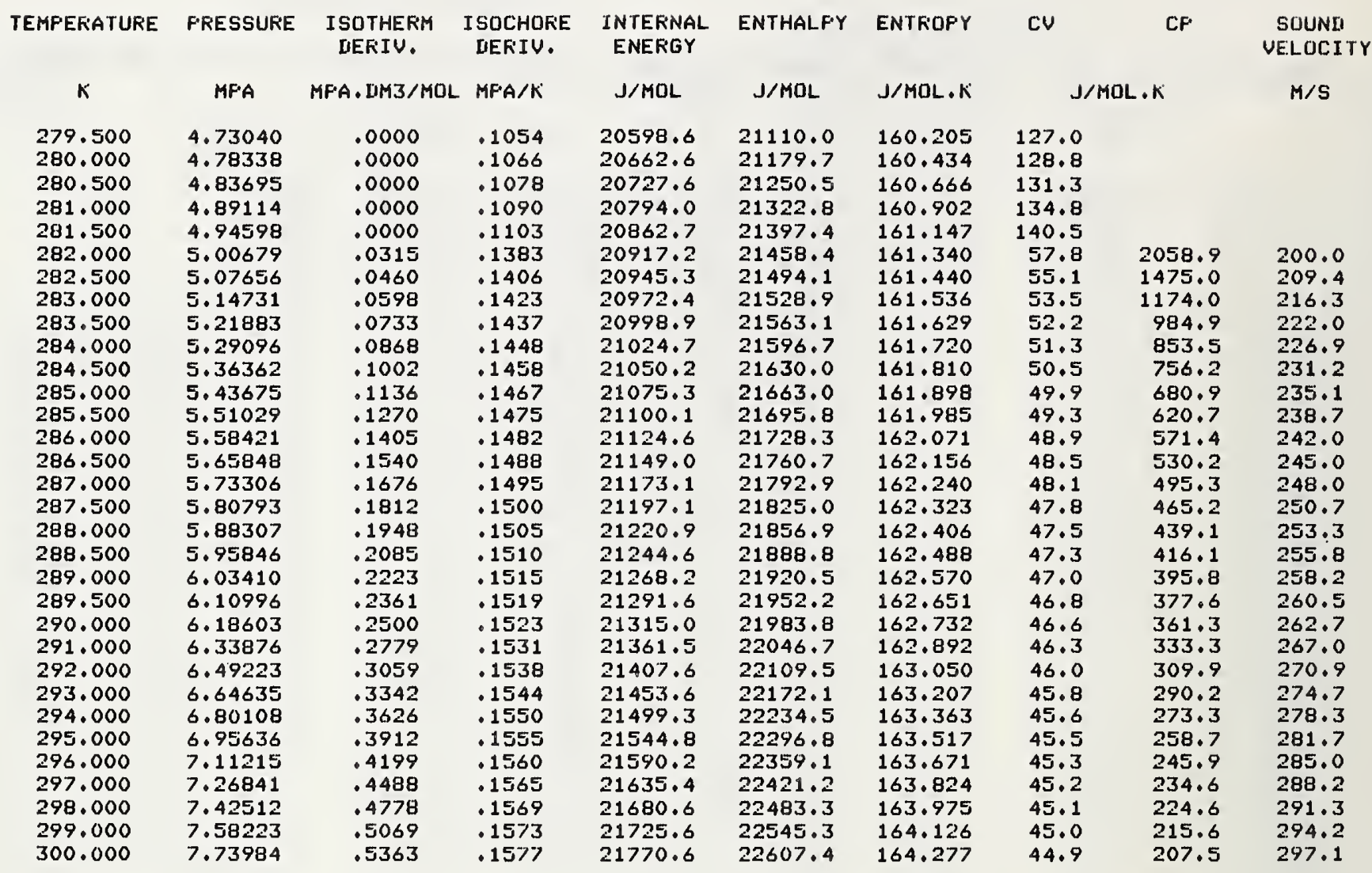


Table CI. (Continued)

$9.50 \mathrm{MOL} / \mathrm{LIM} 3$ ISOCHOFE

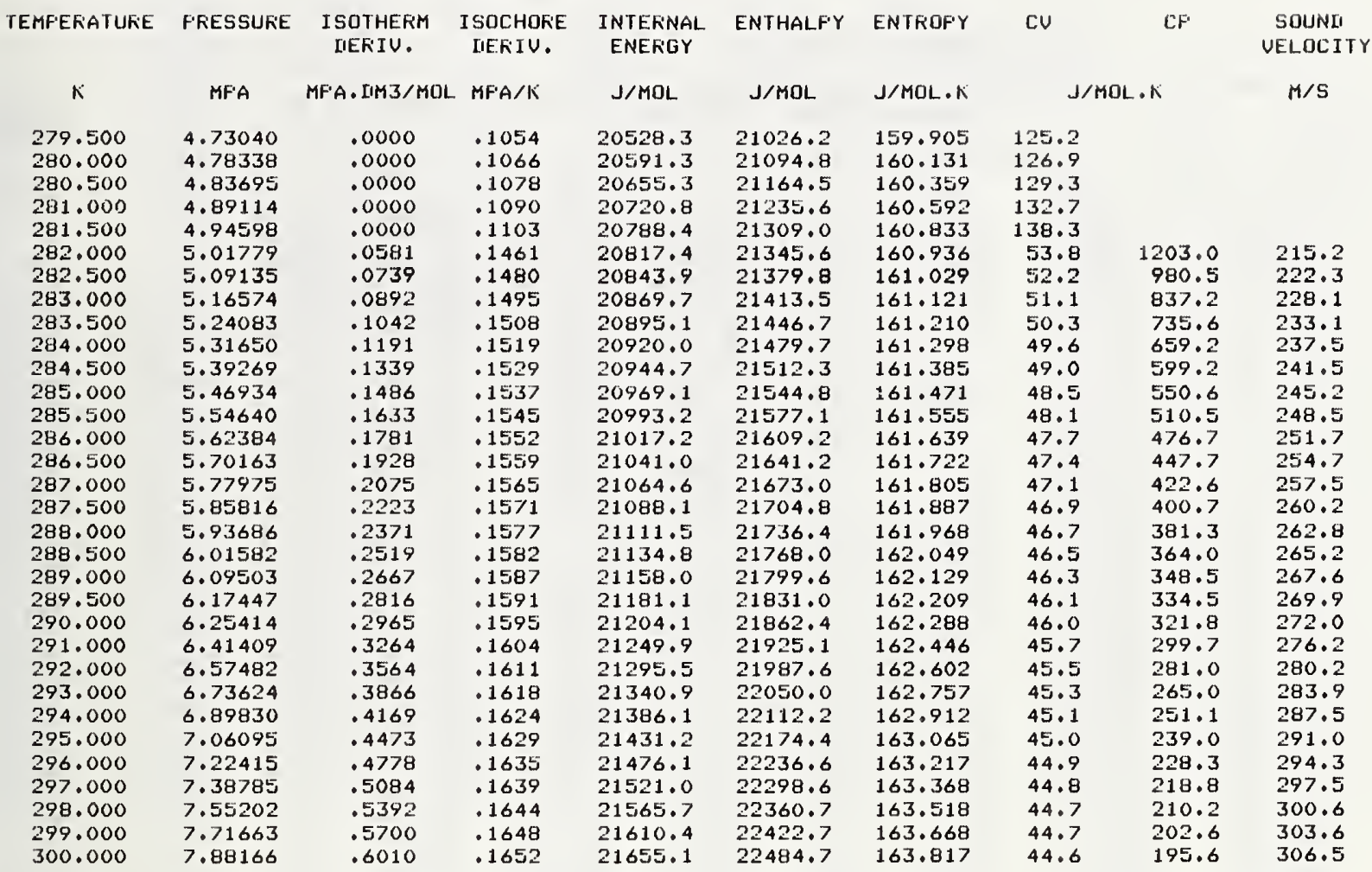


Table CI. (Continued)

9.75 MOL/DM3 ISOCHORE

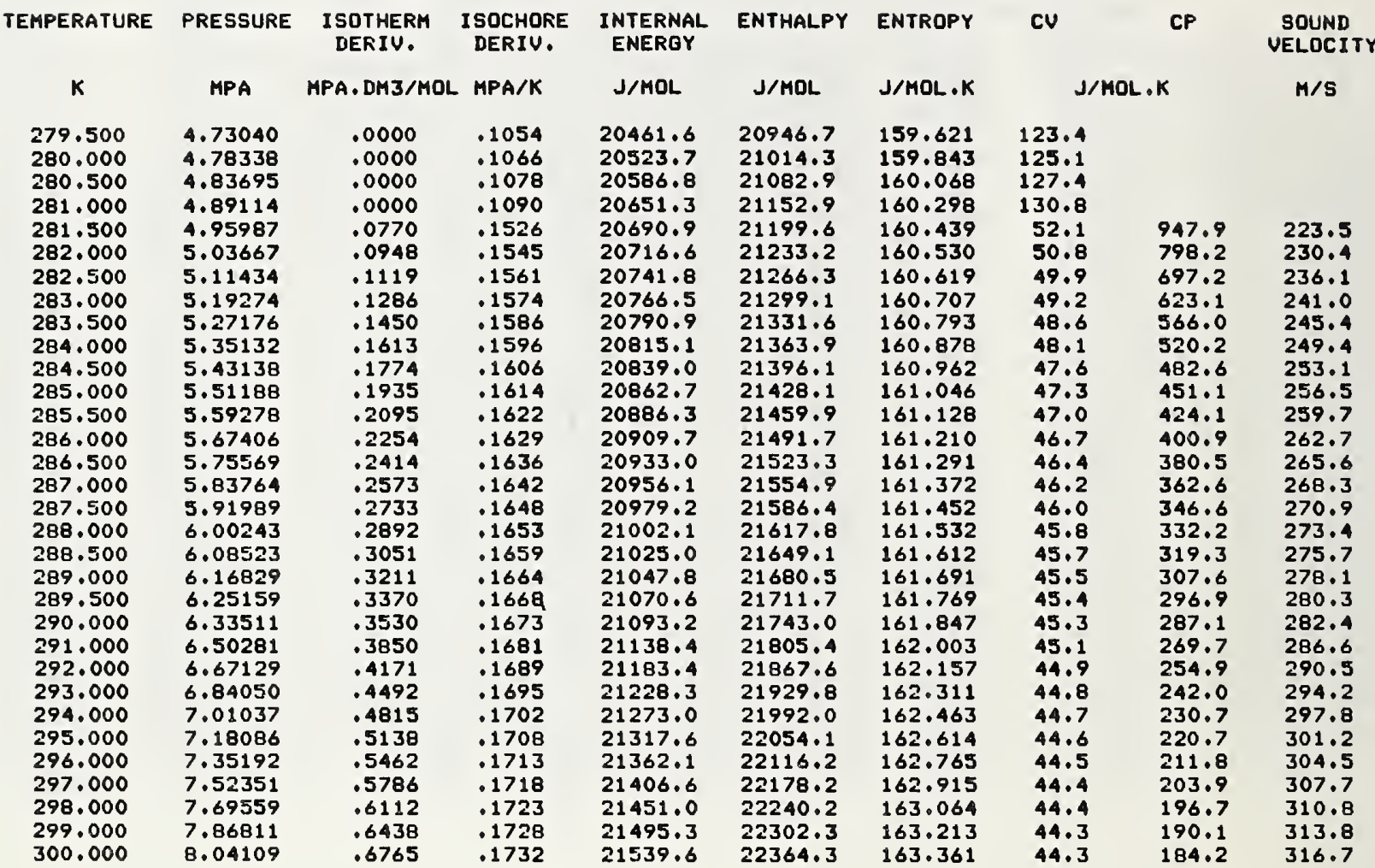


Table CI. (Continued)

$10.00 \mathrm{MOL} / \mathrm{IMM} 3$ ISOCHORE

TEMF'EFIATUFE

ISOTHEFM ISOCHORE INTEFNAL ENTHALFY ENTFIOFY IIERIU.

IIEFIU.

$k$

MF'A

MFA. IMM3/MOL MFA/K

4.73040

.0000

.1054

$4.78338 \quad .0000$

4.83695

.0000

.1066

380.500

281.000

281.500

282.000

282.500

283.000

283.500

284.000

284.500

285.000

285.500

286.000

286.500

287.000

287.500

288.000

288.500

289,000

289.500

290.000

291.000

292.000

293.000

294.000

295.000

296.000

297.000

298.000

299.000

300.000

4.90425

.1047

$.1243 \quad .1619$

$5.06615 \quad .1431 \quad .1635$

$5.14825 \quad .1614 \quad .1649$

$5.23098 \quad .1793 \quad .1660$

$5.31427 \quad .1971$

$5.39806 \quad .2146$

$5.48232 \quad .2321$

$5.56699 \quad .2494$

$5.65206 \quad .2667$

5.73748

5.82325

5.90933

5.99572

6.08238

6.16931

6.25650

6.34393

6.43158

6.60754

6.78430

6.96180

7.13998

7.31879

7.49819

7.67812

7.85857

8.03950

8.22087

.2839

.3011

. 3182

.3353

. 3524

.3695

.3865

.4036

.4207

.4548

.4890

.5231

.5574

.5916

.6259

.6603

.6947

.7291

.7636

.1660

.1681

.1689

.1698

.1705

.1712

.1719

.1735

.1731

.1736

.1741

.1746

.1751

.1755

.1764

.1771

.1778

.1785

.1791

.1797

.1802

.1807

.1812

.1816
CV

CF

SOUNA ENEFIGY

J/MOL
20398.2
20459.4
20521.7
20565.3
20590.2
20614.6
20638.7
20662.6
20686.2
20709.6
20732.9
20756.0
20779.0
20801.9
20824.7
20847.4
20870.0
20892.5
20915.0
20937.5
20959.9
20982.2
21026.8
21071.2
21115.5
21159.7
21203.9
21248.0
21292.1
21336.1
21380.1
21424.0

$J / M O L$

J/MOL.K

$J / M O L \cdot K$

UELOCITY

$20871.2 \quad 159.351$

$20937.8 \quad 159.570$

$21005.4 \quad 159.792$

$21055.7 \quad 159.947$

$21088.7 \quad 160.036$

$21121.2 \quad 160.123$

$21153.6 \quad 160.208$

$21185.7 \quad 160.292$

$21217.6 \quad 160.376$

$21249.4 \quad 160.458$

$21281.1 \quad 160.540$

$21312.7 \quad 160.621$

$21344.2 \quad 160.702$

$21375.6 \quad 160.782$

$21407.0 \quad 160.862$

$21438.3 \quad 160.941$

$21469.6 \quad 161.019$

$21500.8 \quad 161.098$

$21532.0 \quad 161.176$

$21563.1 \quad 161.254$

$21594.2 \quad 161.331$

$21625.4 \quad 161.408$

$21687.5 \quad 161.561$

$21749.6 \quad 161.714$

$21811.7 \quad 161.865$

$21873.7 \quad 162.016$

$21935.8 \quad 162.166$

$21997.8 \quad 162.315$

$22059.9 \quad 162.464$

$22121.9 \quad 162.612$

$22184.0 \quad 162.758$

$22246.1 \quad 162.906$
121.7

123.4

125.7

50.2

49.3

48.5

47.9

47.4

47.0

46.7

46.4

46.1

45.9

45.7

45.5

45.3

45.2

45.0

44.9

44.8

44.7

44.6

44.5

44.4

44.3

44.2

44.1

44.1

44.0

44.0

44.0

44.0
738.0

643.5

575.6

523.8

482.5

448.7

420.4

396.3

375.4

357.1

340.9

326.6

313.6

302.0

291.4

281.7

272.8

264.6

257.1

243.5

231.8

221.4

212.3

204.1

196.7

190.1

184.1

178.6

173.5
$\mathrm{M} / \mathrm{S}$

234.1

240.5

245.9

250.7

255.0

258.9

262.5

265.9

269.0

272.0

274.9

277.6

280.2

282.6

285.0

287.4

289.6

291.8

293.9

297.9

301.7

305.4

308.9

312.3

315.6

318.7

321.8

324.8

327.8 
Table CI. (Continued)

10.25 MOL/LIM3 ISOCHORE

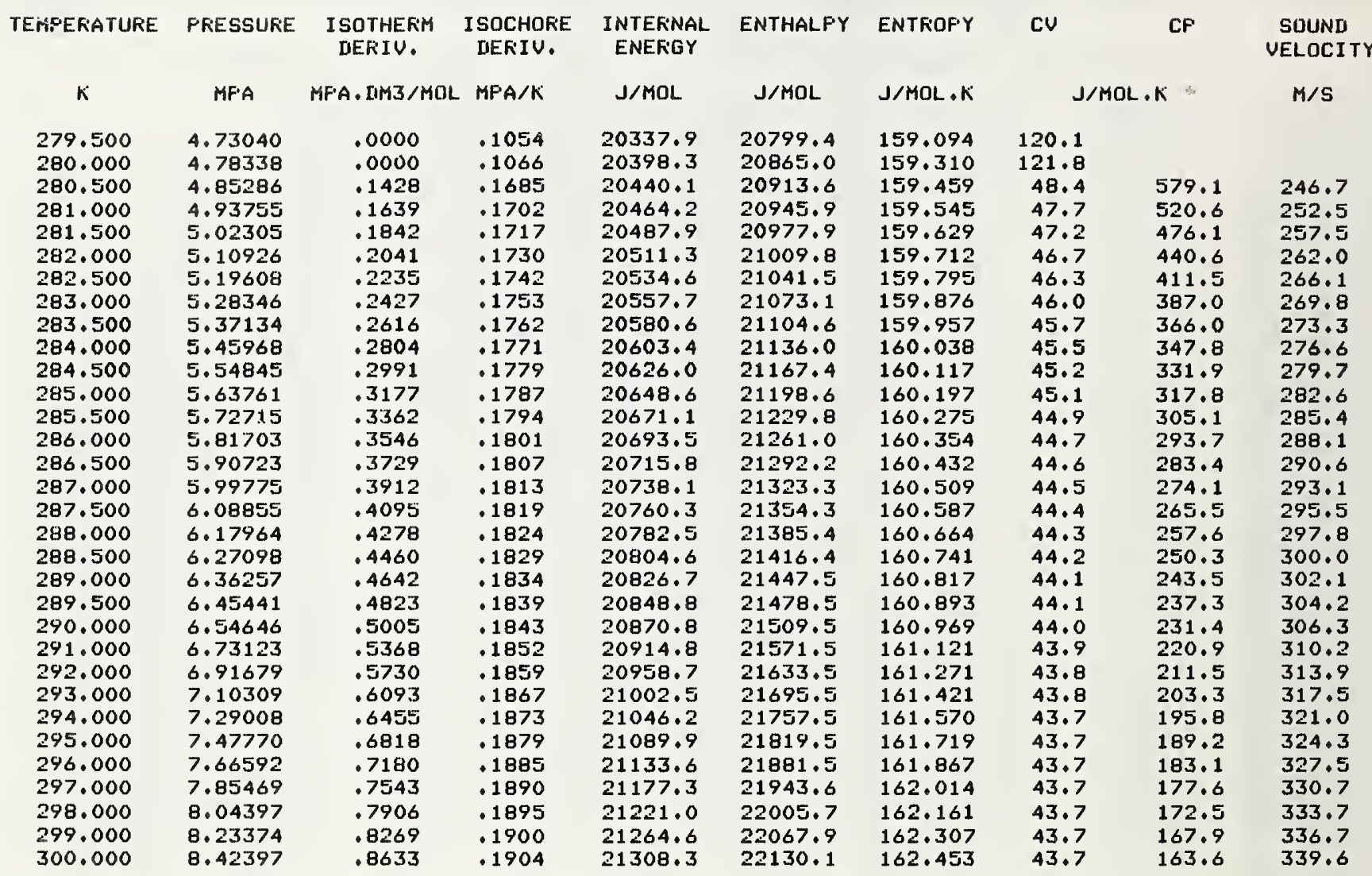


Table CI. (Continued)

10.50 MOL/UM3 ISOCHORE

\begin{tabular}{|c|c|c|c|c|c|c|c|c|c|}
\hline TEMPERATURE & F'RESSURE & $\begin{array}{l}\text { ISOTHERM } \\
\text { IIERIV. }\end{array}$ & $\begin{array}{l}\text { ISOCHOKE } \\
\text { DEFIU. }\end{array}$ & $\begin{array}{c}\text { INTEFNAL } \\
\text { ENERGY }\end{array}$ & ENTHALFY & ENTROPY & $\mathrm{cU}$ & $\mathrm{CF}$ & $\begin{array}{l}\text { SOUND } \\
\text { VELOCITY }\end{array}$ \\
\hline$\kappa$ & MFA & $M P A, I M 3 / M O L$ & $M P A / K$ & J/MOL & $J / M O L$ & $J / M O L \cdot K$ & \multicolumn{2}{|c|}{$J / M O L \cdot K$} & $M / S$ \\
\hline 279.500 & 4.73040 & .0000 & .1054 & 20280.5 & 20731.0 & 158.849 & $118 \cdot 6$ & & \\
\hline 280.000 & 4.80802 & .1928 & .1778 & 20314.9 & 20772.8 & 158.972 & 46.8 & 463.1 & 260.9 \\
\hline 280.500 & 4.89731 & .2151 & .1794 & 20338.2 & 20804.6 & 159.055 & 46.2 & 426.7 & 266.0 \\
\hline 281.000 & 4.98734 & .2368 & .1808 & 20361.2 & 20836.2 & 159.137 & 45.8 & 397.5 & 270.6 \\
\hline 281.500 & 5.07803 & $\cdot 2580$ & .1820 & 20384.0 & 20867.6 & 159.218 & 45.5 & 373.3 & 274.7 \\
\hline $\begin{array}{l}282.000 \\
282.500\end{array}$ & $\begin{array}{l}5.16932 \\
5.26114\end{array}$ & $\begin{array}{r}.2788 \\
.2993\end{array}$ & $\begin{array}{r}.1831 \\
.1841\end{array}$ & $\begin{array}{l}20406.7 \\
20429.2\end{array}$ & $\begin{array}{l}20899.0 \\
20930.3\end{array}$ & $\begin{array}{l}159.299 \\
159.379\end{array}$ & $\begin{array}{l}45 \cdot 2 \\
44.9\end{array}$ & $\begin{array}{l}352.8 \\
335.2\end{array}$ & $\begin{array}{l}278.6 \\
282.1\end{array}$ \\
\hline 283.000 & 5.35345 & .3196 & .1851 & 20451.6 & 20961.5 & 159.458 & 44.7 & 319.8 & 285.5 \\
\hline 283.500 & 5.44622 & .3398 & .1860 & 20473.9 & 20992.6 & 159.537 & 44.5 & 306.3 & $288 \cdot 6$ \\
\hline 284.000 & 5.53941 & .3597 & . 1868 & 20496.2 & 21023.7 & 159.615 & 44.4 & 294.2 & 291.6 \\
\hline 284.500 & 5.63300 & .3796 & .1875 & $20518 \cdot 3$ & 21054.8 & 159.693 & 44.2 & $283 \cdot 3$ & 294.4 \\
\hline 285.000 & 5.72695 & .3993 & .1883 & 20540.4 & 21085.8 & 159.771 & 44.1 & 273.5 & 297.1 \\
\hline 285.500 & 5.82125 & .4190 & .1889 & 20562.4 & 21116.8 & 159.848 & 44.0 & 264.6 & 299.7 \\
\hline 286.000 & 5.91588 & .4386 & .1896 & 20584.4 & 21147.8 & 159.925 & 43.9 & 256.5 & 302.2 \\
\hline 286.500 & 6.01083 & .4581 & .1902 & 20606.3 & 21178.8 & 160.001 & 43.8 & 249.0 & $304 \cdot 6$ \\
\hline 287.000 & 6.10606 & .4775 & .1908 & 20628.2 & 21209.7 & 160.078 & 43.7 & $242 \cdot 1$ & 307.0 \\
\hline 287.500 & 6.20158 & .4969 & .1913 & 20650.0 & 21240.7 & 160.154 & 43.7 & 235.7 & 309.2 \\
\hline 288.000 & 6.29736 & .5163 & .1918 & 20671.9 & $21271 \cdot 6$ & 160.229 & 43.6 & 229.8 & 311.4 \\
\hline $28 \mathrm{~B} .500$ & 6.39340 & .5356 & .1923 & 20693.7 & 21302.6 & 160.305 & 43.6 & 224.3 & 313.5 \\
\hline 289.000 & 6.48967 & .5549 & .1928 & 20715.4 & 21333.5 & 160.380 & 43.5 & $219 \cdot 1$ & $315 \cdot 6$ \\
\hline 289.500 & 6.58619 & .5742 & .1932 & 20737.2 & 21364.4 & 160.456 & 43.5 & $214 \cdot 3$ & 317.6 \\
\hline 290.000 & 6.68292 & .5934 & .1937 & 20758.9 & 21395.4 & 160.531 & 43.4 & 209.7 & 319.6 \\
\hline 291.000 & 6.87702 & .6318 & .1945 & 20802.3 & 21457.3 & 160.680 & 43.4 & 201.4 & 323.4 \\
\hline 292.000 & 7.07191 & .6702 & .1953 & 20845.7 & 21519.2 & 160.829 & $43 \cdot 3$ & 194.0 & 327.0 \\
\hline 293.000 & 7.26754 & .7085 & .1960 & 20889.0 & $21581 \cdot 1$ & 160.977 & 43.3 & 187.4 & 330.5 \\
\hline 294.000 & 7.46384 & .7468 & .1966 & 20932.3 & 21643.2 & 161.124 & 43.3 & 181.4 & 333.9 \\
\hline 295.000 & 7.66078 & .7850 & .1972 & 20975.6 & 21705.2 & 161.271 & $43 \cdot 3$ & 175.9 & 337.2 \\
\hline $\begin{array}{l}296.000 \\
297.000\end{array}$ & $\begin{array}{l}7.85830 \\
8.05638\end{array}$ & $\begin{array}{r}.8233 \\
.8615\end{array}$ & $\begin{array}{r}.1978 \\
.1983\end{array}$ & $\begin{array}{l}21018.9 \\
21062.2\end{array}$ & $\begin{array}{l}21767 \cdot 3 \\
21829 \cdot 5\end{array}$ & $\begin{array}{l}161.418 \\
161.564\end{array}$ & $\begin{array}{l}43 \cdot 3 \\
43 \cdot 3\end{array}$ & $\begin{array}{l}170.9 \\
166.3\end{array}$ & $\begin{array}{l}340 \cdot 3 \\
343 \cdot 4\end{array}$ \\
\hline 298.000 & 8.25498 & .8997 & .1988 & 21105.5 & 21891.7 & 161.710 & 43.3 & 162.1 & 346.4 \\
\hline 299.000 & 8.45407 & .9379 & .1993 & 21148.9 & 21954.0 & 161.855 & 43.3 & 158.2 & 349.4 \\
\hline 300.000 & 8.65362 & .9762 & .1998 & 21192.2 & 22016.4 & 162.000 & 43.4 & 154.6 & 352.2 \\
\hline
\end{tabular}


Table CIIa. Saturation properties of ethylene as a function of temperature

COEXISTING FHASE FROFERTIES, LIQUII

\begin{tabular}{|c|c|c|c|c|c|c|c|c|c|}
\hline TEMFEFATURE & FKESSURE & IENSITY & $\begin{array}{l}\text { LATENT } \\
\text { HEAT }\end{array}$ & $\begin{array}{c}\text { INTE.FNAL } \\
\text { ENEFIGY }\end{array}$ & ENTHALFY & ENTFOFY & CV & $C F$ & $\begin{array}{l}\text { VEL_OCITY } \\
\text { OF SOUNA }\end{array}$ \\
\hline$\kappa$ & MF'A & MOL/IIM3 & J/MOL & $\mathrm{J} / \mathrm{MOL}$ & $J / M O L$ & J/MOL.バ & \multicolumn{2}{|c|}{$J / M O L \cdot K K$} & $M / S$ \\
\hline 279.700 & 4.75152 & 10.481 & 3216.9 & 20308.5 & 20761.9 & 158.952 & 47.3 & 502.2 & 255.9 \\
\hline 279.800 & 4.76211 & 10.440 & 3168.9 & 20329.8 & 20785.9 & 159.035 & 47.6 & 519.9 & 253.6 \\
\hline 279.900 & 4.77273 & 10.398 & 3120.0 & 20351.4 & 20810.4 & 159.119 & 47.8 & 539.1 & 251.3 \\
\hline 280.000 & 4.78338 & 10.356 & 3069.9 & 20373.4 & 20835.3 & 159.204 & 48.1 & 560.0 & 249.0 \\
\hline 280.100 & 4.79404 & 10.312 & 3018.8 & 20395.8 & 20860.7 & 159.291 & 48.4 & 583.0 & $246+6$ \\
\hline 280.200 & 4.80473 & 10.267 & 2966.4 & 20418.7 & 20886.7 & 159.380 & 48.8 & 608.2 & 244,2 \\
\hline 280.300 & 4.81545 & 10.221 & 2912.7 & 20442.1 & 20913.2 & 159.471 & 49.1 & 636.0 & 241.7 \\
\hline $280+400$ & 4.82619 & 10.174 & 2957.5 & 20465.9 & $20940 \cdot 3$ & 159.564 & 49.5 & 666.9 & 239.2 \\
\hline 280.500 & 4.83695 & 10.125 & 2800.8 & 20490.4 & 20968.1 & 159.659 & 49.8 & 701.5 & 236.7 \\
\hline 280.600 & 4.84774 & 10.074 & 2742.4 & 20515.5 & 20996.7 & 159.757 & 50.3 & 740.2 & $234 \cdot 1$ \\
\hline 280.700 & 4.85855 & 10.022 & 2682.1 & 20541.2 & 21026.0 & 159.858 & 50.7 & 784.1 & 231.4 \\
\hline 280.800 & 4.86939 & 9.969 & 2619.7 & 20567.7 & 21056.2 & 159.961 & 51.1 & 834.1 & 228.7 \\
\hline 280.900 & 4.88026 & 9.913 & 2555.0 & 20595.1 & 21087.4 & 160.069 & 51.6 & 891.6 & 225.9 \\
\hline 281.000 & 4.89114 & 9.854 & 2487.8 & 20623.3 & 21119.7 & 160.180 & 52.2 & 958.3 & 223.0 \\
\hline 281.100 & 4.90206 & 9.793 & 2417.7 & 20652.7 & 21153.2 & 160.295 & 52.8 & 1036.8 & 220.0 \\
\hline 281.200 & 4.91300 & 9.730 & 2344.3 & $20683+2$ & 21188.1 & 160.415 & 53.4 & 1130.3 & 216.9 \\
\hline 281.300 & 4.92397 & 9.662 & 2267.1 & $20 / 15+1$ & 21224.7 & 160.541 & 54.1 & 1243.3 & 213.7 \\
\hline 281.400 & 4.93496 & 9.591 & 2185.5 & 20748.6 & 21263.1 & 160.674 & 54.9 & 1382.8 & $210 \cdot 3$ \\
\hline 281.500 & 4.94598 & 9.515 & 2098.6 & 20784.0 & 21303.8 & 160.814 & 55.8 & 1558.7 & $206 \cdot 8$ \\
\hline 281.600 & 4.95703 & 9.434 & 2005.5 & 20821.7 & 21347.1 & 160.964 & 56.8 & 1787.1 & 203.0 \\
\hline 281.700 & 4.96810 & 9.345 & 1904.7 & 20862.2 & 21393.8 & 161.125 & 58.0 & 2094.6 & 199.0 \\
\hline 281.800 & 4.97921 & 9.248 & 1794.0 & $20906+3$ & 21444.8 & 161.302 & 59.5 & 2528.8 & 194.7 \\
\hline 281.900 & 4.99034 & 9.138 & 1670.2 & 20955.3 & 21501.4 & 161.499 & 61.3 & 3183.9 & 189.9 \\
\hline $282+000$ & 5.00150 & 9.012 & 1528.0 & 21011.1 & $21566+1$ & 161.724 & 63.6 & 4273.4 & 184.4 \\
\hline 282.100 & 5.01270 & 8.861 & 1357.2 & 21077.6 & 21643.3 & 161.993 & 66.8 & 6396.2 & 178.0 \\
\hline 282.200 & 5.02393 & 8.662 & 1134.4 & 21163.6 & 21743.6 & 162.344 & 72.2 & 12021.7 & 169.5 \\
\hline $282 \cdot 300$ & 5.03519 & 8.330 & 766.1 & 21305.2 & 21909.6 & 162.928 & 85.6 & 50818.0 & 154.5 \\
\hline 282.345 & 5.04030 & 7.634 & 0.0 & $21610+1$ & 22270.3 & 164.203 & & & 0.0 \\
\hline
\end{tabular}


Table CIIb. Saturation properties of ethylene as a function of temperature

COEXISTING FHASE FROFERTIES, VAFOF

\begin{tabular}{|c|c|c|c|c|c|c|c|c|c|}
\hline TEMF'ER'ATURE & PRESSURE & IIENSITY & $\begin{array}{l}\text { LATENT } \\
\text { HEAT }\end{array}$ & $\begin{array}{l}\text { INTEF'NAL } \\
\text { ENEFGY }\end{array}$ & ENTHALFY & ENTROFY & cu & $C F$ & $\begin{array}{l}\text { UELOCITY } \\
\text { OF SOUNI }\end{array}$ \\
\hline$k$ & MF'A & MOL/IIM3 & $\mathrm{J} / \mathrm{MOL}$ & J/MOL & J/MOL & $\mathrm{J} / \mathrm{MOL} \cdot \mathrm{K}$ & \multicolumn{2}{|c|}{$J / M O L \cdot K$} & $M / S$ \\
\hline 281.400 & 4.93496 & 5.720 & 2185.4 & 22585.8 & 23448.5 & 168.440 & 62.7 & 2253.7 & 199.0 \\
\hline 281.500 & 4.94598 & 5.792 & 2098.6 & 22548.4 & 23402.4 & 168.269 & 63.6 & 2545.1 & 197.7 \\
\hline $\begin{array}{l}281.600 \\
281.700 \\
281.800\end{array}$ & $\begin{array}{l}4.95703 \\
4.96810 \\
4.97921\end{array}$ & $\begin{array}{l}5.869 \\
5.953 \\
6.046\end{array}$ & $\begin{array}{l}2005.5 \\
1904.7 \\
1794.0\end{array}$ & $\begin{array}{l}22508.0 \\
22464.0 \\
22415.2\end{array}$ & $\begin{array}{l}23352.7 \\
23298.5 \\
23238.8\end{array}$ & $\begin{array}{l}168.086 \\
167.887 \\
167.668\end{array}$ & $\begin{array}{l}64.7 \\
65.9 \\
67.4\end{array}$ & $\begin{array}{l}2921.0 \\
3423.2 \\
4125.8\end{array}$ & $\begin{array}{l}196.2 \\
194.5 \\
192.4\end{array}$ \\
\hline 281.900 & 4.99034 & 6.151 & 1670.2 & 22360.3 & 23171.6 & 167.424 & 69.2 & 5173.2 & 190.0 \\
\hline $\begin{array}{l}282.000 \\
282.100\end{array}$ & $\begin{array}{l}5.00150 \\
5.01270\end{array}$ & $\begin{array}{l}6.273 \\
6.420\end{array}$ & 1528.0 & 22296.7 & 23094.0 & 167.142 & 71.5 & 6887.8 & 186.9 \\
\hline $282 \cdot 100$ & 5.01270 & 6.420 & 1357.2 & 22219.7 & 23000.5 & 166.804 & 74.7 & 10155.8 & 182.7 \\
\hline 282.200 & 5.02393 & 6.614 & 1134.4 & 22118.4 & 22878.0 & 166.364 & 80.0 & 18527.2 & 176.2 \\
\hline 282.300 & 5.03519 & 6.940 & 766.1 & 21950.2 & 22675.7 & 165.641 & 93.0 & 72255.4 & 162.1 \\
\hline 282.345 & 5.04030 & 7.634 & 0.0 & 21610.2 & 22270.4 & 164.203 & & & 0.0 \\
\hline
\end{tabular}


Table CIIIa. Saturation properties of ethylene as a funtion of pressure

COEXISTING PHASE FROFERTIES, LIRUII

\begin{tabular}{|c|c|c|c|c|c|c|c|c|c|}
\hline EMFERATURE & F'RESSURE & IIENS I TY & $\begin{array}{l}\text { LATENT } \\
\text { HEAT }\end{array}$ & $\begin{array}{c}\text { INTERNAL } \\
\text { ENERGY }\end{array}$ & ENTHALFY & ENTFOFY & CU & CP & $\begin{array}{l}\text { UELOCITY } \\
\text { OF SOUNI }\end{array}$ \\
\hline к & MF'A & MOL/IIM3 & $\mathrm{J} / \mathrm{MOL}$ & $\mathrm{J} / \mathrm{MOL}$ & \rfloor$/ M O L$ & J/MOL.K & \multicolumn{2}{|c|}{ J/MOL.K゙ } & $M / S$ \\
\hline 279.686 & 4.75000 & 10.487 & 3223.7 & 20305.5 & 20758.4 & 158.941 & 47.3 & 499.8 & 256.2 \\
\hline 279.780 & 4.76000 & 10.443 & 3178.6 & 20325.5 & 20781.1 & 159.018 & 47.5 & 516.2 & 254.0 \\
\hline 279.874 & 4.77000 & 10.409 & 3132.7 & 20345.8 & 20804.0 & 159.097 & 47.8 & 534.0 & 251.9 \\
\hline 279.968 & 4.78000 & 10.369 & 3085.9 & 20366.4 & 20827.4 & 159.177 & 48.0 & 553.2 & 249.7 \\
\hline 280.062 & 4.79000 & 10.329 & 3038.3 & 20387.3 & 20851.0 & 159.258 & 48.3 & 574.0 & 247.5 \\
\hline 280.156 & 4.80000 & 10.287 & 2989.7 & 20408.5 & 20875.1 & 159.340 & 48.6 & 596.7 & 245.3 \\
\hline $\begin{array}{l}280.249 \\
280.342\end{array}$ & $\begin{array}{l}4.81000 \\
4.82000\end{array}$ & $\begin{array}{l}10.245 \\
10.201\end{array}$ & $\begin{array}{l}2940.2 \\
2889.5\end{array}$ & $\begin{array}{l}20430.1 \\
20452.1\end{array}$ & $\begin{array}{l}20899.6 \\
20924.6\end{array}$ & $\begin{array}{l}159.424 \\
159.510\end{array}$ & $\begin{array}{l}48.9 \\
49.3\end{array}$ & $\begin{array}{l}621.5 \\
648.7\end{array}$ & $\begin{array}{l}243.0 \\
240.7\end{array}$ \\
\hline $\begin{array}{l}280.342 \\
280.435\end{array}$ & $\begin{array}{l}4.82000 \\
4.83000\end{array}$ & $\begin{array}{l}10.201 \\
10.156\end{array}$ & 2837.6 & 20474.5 & $\begin{array}{l}20924.0 \\
20950.1\end{array}$ & $\begin{array}{l}159.510 \\
159.597\end{array}$ & $\begin{array}{l}48.3 \\
49.6\end{array}$ & $\begin{array}{l}648.7 \\
678.7\end{array}$ & 238.3 \\
\hline 280.528 & 4.84000 & 10.111 & 2784.5 & 20497.4 & 20976.1 & 159.687 & 50.0 & 712.0 & 236.0 \\
\hline 280.621 & 4.85000 & 10.064 & 2729.9 & 20520.8 & 21002.7 & 159.778 & 50.3 & 748.9 & 233.5 \\
\hline 280.713 & 4,86000 & 10.015 & 2673.9 & 20544.7 & 21030.0 & 159.871 & 50.7 & 790.4 & 231.0 \\
\hline 280.806 & 4.87000 & 9.965 & 2616.1 & 20569.2 & 21057.9 & 159.967 & 51.2 & 837.1 & 228,5 \\
\hline 280.898 & 4.88000 & 9.914 & 2556.6 & 20594.4 & 21086.6 & 160.066 & 51.6 & 890.1 & 225.9 \\
\hline 280.989 & 4.89000 & 9.861 & 2495.0 & 20620.3 & 21116.2 & 160.168 & 52.1 & 950.8 & 223.3 \\
\hline 281.081 & 4.90000 & 9.805 & 2431.1 & 20647.0 & 21146.8 & 160.273 & 52.6 & 1021.0 & 220.5 \\
\hline 281.173 & 4.91000 & 9.747 & 2364.7 & 20674.7 & 21178.4 & 160.382 & 53.2 & 1102.9 & 217.7 \\
\hline 281.264 & 4.92000 & 9.687 & 2295.4 & 20703.4 & 21211.3 & 160.495 & 53.9 & 1199.9 & 214.8 \\
\hline 281.355 & 4.93000 & 9.624 & 2222.8 & 20733.3 & 21245.5 & 160.613 & 54.5 & 1316.1 & 211.8 \\
\hline 281.446 & 4.94000 & 9.557 & 2146.4 & 20764.5 & 21281.4 & 160.737 & 55.3 & 1458.0 & 208.7 \\
\hline 281.536 & 4.95000 & 9.486 & 2065.5 & 20797.4 & 21319.2 & 160.867 & 56.2 & 1634.7 & 205.4 \\
\hline 281.627 & 4.96000 & 9.411 & 1979.3 & 20832.2 & $21359 \cdot 3$ & 161.006 & 57.1 & 1860.5 & 202.0 \\
\hline 281.717 & 4.97000 & 9.329 & 1886.5 & 20869.5 & 21402.2 & 161.155 & 58.3 & 2158.1 & 198.3 \\
\hline 281.807 & 4.98000 & 9.240 & 1785.6 & 20909.7 & 21448.6 & 161.315 & 59.6 & 2566.6 & 194.3 \\
\hline 281.897 & 4.99000 & 9.142 & 1674.2 & 20953.7 & 21499.6 & 161.492 & 61.2 & 3159.1 & 190.0 \\
\hline 281.987 & 5.00000 & 9.031 & 1548.5 & 21003.1 & 21556.8 & 161.691 & 63.2 & 4087.2 & 185.2 \\
\hline 282.076 & 5.01000 & 8.900 & 1401.8 & 21060.3 & 21623.2 & 161.923 & 65.9 & 5722.8 & 179.7 \\
\hline 282.165 & 5.02000 & 8.739 & 1220.9 & 21130.2 & 21704.7 & 162.208 & 69.9 & 9256.3 & 172.8 \\
\hline 282.254 & 5.03000 & 8.514 & 969.4 & 21227.0 & 21817.8 & 162.604 & 77.3 & 21237.6 & 163.0 \\
\hline 282.343 & 5.04000 & 7.909 & 301.1 & 21486.9 & 22124.2 & 163.686 & 127.5 & $1.8003+6$ & 128.3 \\
\hline 282.345 & 5.04030 & 7.634 & 0.0 & 21610.1 & 22270.3 & 164.203 & & & 0.0 \\
\hline
\end{tabular}


Table CIIIb. Saturation properties of ethylene as a function of pressure

COEXISTING FHASE F'ROFEFTIES, VAFOR

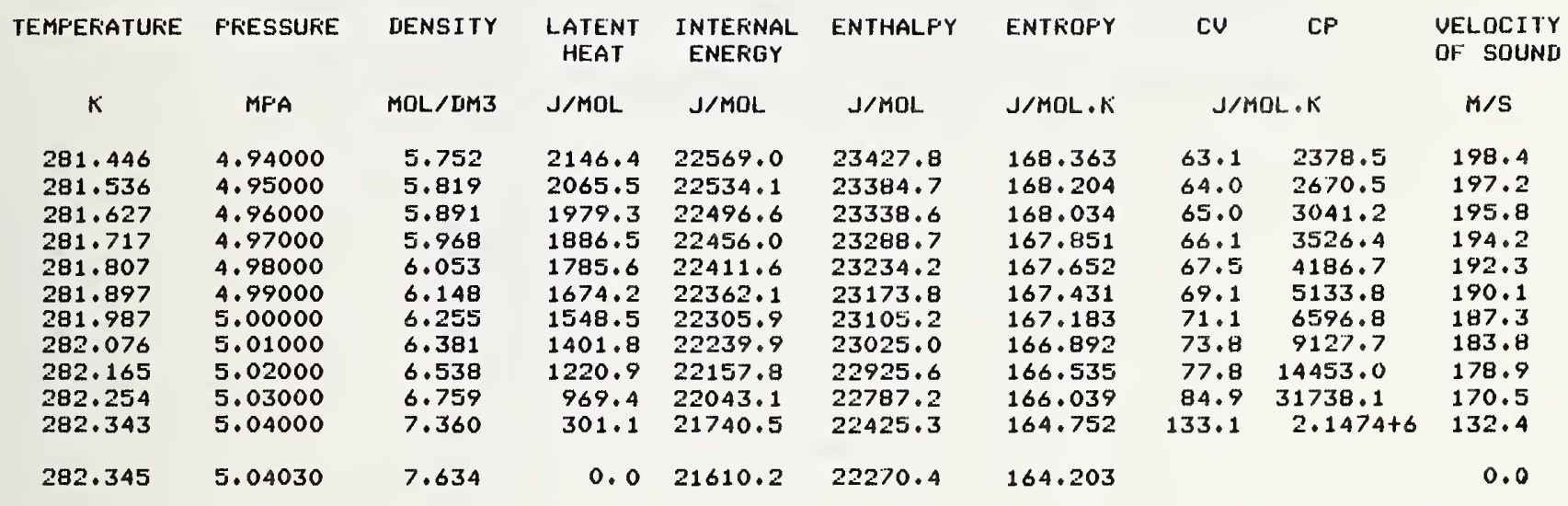


APPENDIX D

Listing of Computer Program for Table Generation 
IMFLICIT FEAL*B(A-H,O-Z)

C FEAL ANAMES, QNAMES

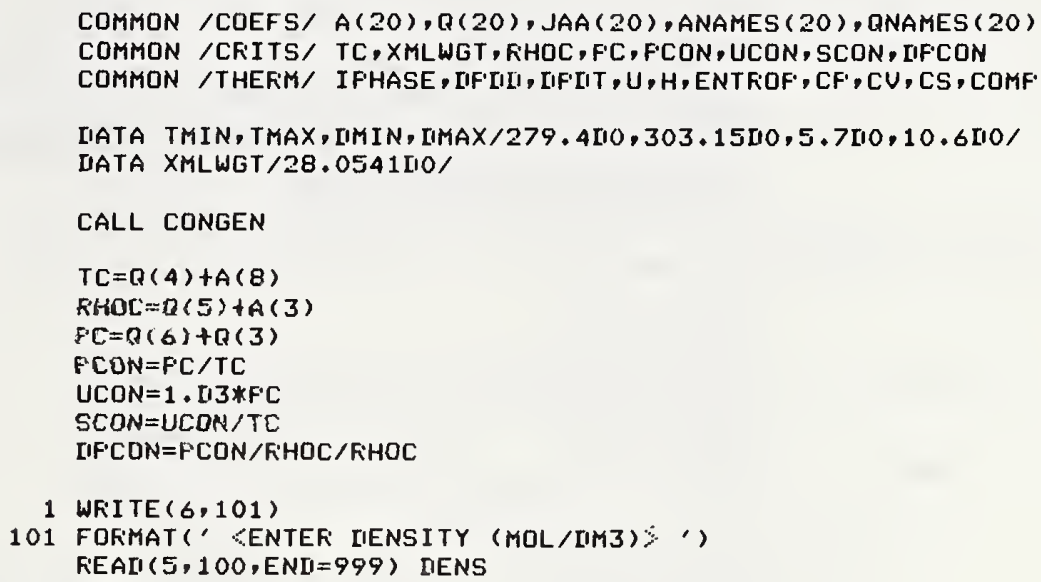

IF (IIENS, LE, DMAX, ANI, IENS, GE, IMIN) GO TO 11

WFITE $(6,102)$

102 FOKMAT ( ' * * THE DENSITY INFUT IS OUTSIIIE FANGE OF ', 1 'ERUATION' 'S UALIIITY**'') GO TO 1

C

11 IIIF IND $=0$

WRI I TE $(6,130)$

130 FORMAT(" ENTER START TEMF', STOF' TEMF', \& INCREMENT') FEAII $(5,100)$. T1,T2,TINC IF (T1.LT.TMIN) T1=TMIN 
C THIS F'ROGFAM CALCULATES THE COEXISTENCE F'ROFEFTIES

C INDEFENIIENT VAFIABLE IS TEMFERATURE

C

IMF LICIT FEAL*B (A-H,O-Z)

REAL TITLE

FEAL ANAMES, RNAMES

COMMON/COEFS/A (20), $Q(20)$, JAA (20), ANAMES (20), RNAMES (20)

COMMON/SATUK/IUAF, HEAT, KHHOL, FHOG

COMMON/THEFIM/I FHASE, LIF'III, LF LIT, U, H, S, CF, CU, CS, COMF

COMMON/CFI TS/TC, XMLWGT, K'HOC, F'C, F'CON , UCON, SCON, IJFCON

LIATA IIMIN, LIMAX/5.510,10.5110/

C

IJATA XMLWGT, TMIN/28,0541[10,279.71.10/

CALL CONGEN

$Q(3)=A(8) *(R(7)$

$T C=Q(4)+A(8)$

$\mathrm{F} H O C=Q(5)+A(3)$

$F C=R(6)+Q(3)$

$\mathrm{F} \cdot \mathrm{CON}=\mathrm{F} \cdot \mathrm{C} / \mathrm{TC}$

$U C O N=1 \cdot[13 * F C$

SCON=UCON/TC

c

IFFCON=F'CON/FHOC $/$ FHOC

WFI I TE $(6,333)$

333 FOFMAT ( "ENTEF SELECTION FAFAMETEF.....1=LIR,-1=VAF’'

REALI $(5,1)$ IUAF

IF (IVAF'.EQ, -1). TITLE='VAF'OR' '

IF (IVAF.EQ.1) TITLE='LIQUII'

IF (IVAF, EQ, -1) TMIN=281.4

201 FOKMAT ( $1 H 1 / 1 / /, 4 X$,'COEXISTING FHASE F'OF'EFTIES, ',A6//4X,' TEMFEFAT 1UF'E', $2 X, ' F ' F E S S U F$ DENSITY LATENT INTEKNAL ENTHALF'Y',3X, 2 'ENTROFY CU CF' UELOCITY'/39X,'HEAT ENEKGY',38X, 3' OF SOUNL'//8X,'K MFA MOL/LM3 J/MOL J/MOL ',

c $\left.43 X_{1}, J / M O L \quad J / M O L \cdot K \quad J / M O L \cdot K \quad M / S, /\right)$ IF IN $=0$

WRITE $(6,3.34)$

334 FOFMAT (' $\angle E N T E R$ STAFT TEMF, ,STOF 'TEMF', \& INCFEMENT $\%$ REALI $(S, 1)$ T1,T2, TINC

IF (T1.LT, TMIN) T1=TMIN

WRITE $(6,201)$ TITLE

$T=T 1-T I N C$

$100 \quad T=T+T$ INC

IF (T.GT.T2) IFIN=1

IF ( T, GE.TC ) IFIN=2

$I F(T, G E \cdot T C) \quad T=T C-1 \cdot[1-14$

II $=\mathrm{F}$ HOC

CALL THEFMO $(O, T, I, F, F, T H E T A)$

IF (IFIN.EQ.2) HEAT $=0$. TIO $^{\circ}$

IF (IFIN.EQ, 2) WFITE (6,202) T,FC, FHOC, HEAT, U,H,S, HEAT

202 FOKMAT(1X/F13.3,F11.5,F10.3,F10.1,F9.1,F 10.1,F11.3,17X,F9.1//// $)$ IF(T.LT.TC . ANI. CF.LT.99999.99) WFITE $(6,203)$

$1 T, F, I, H E A T, U, H, S, C U, C F, C S$

203 FOKMAT(F13.3,F11.5,F10.3,F10.1,F9.1,F10.1,F11,3,F8.1,F9.1,F9.1) I I CF $=0$

IF(T,LT,TC , ANEI, CF',GT.99999,99,ANI,IFIN,NE,2) IICF:=1

IF (IICF',EQ.1) ICF=ILOG $10\left(\mathrm{CF}^{\circ}\right)$

IF ( I ICF'.EQ. 1) $\quad C F=C F / 10,110 * * I C F$

IF (I ICF' EQ. 1) WFI I TE $(6,204) \mathrm{T}, \mathrm{F}^{\prime}, \mathrm{IH}, \mathrm{HEAT}, \mathrm{U}, \mathrm{H}, \mathrm{S}, \mathrm{CV}, \mathrm{CF}, \mathrm{ICF}, \mathrm{CS}$

204 FOKMAT (F13.3,F11,5,F10.3,F10.1,F9,1,F10,1,F10.3,F8,1,F9,4, ' +', I1

1

$, F B .1)$

C IF (IFIN.EQ.0) GO TO 100

WFI I TE $(6,210)$

210 FOFMAT(', $20(/))$ STOF

1 FOKMAT（）

ENT 
C 1. NOT ON THE SATUFATION CUFIVE (ISAT=0) :

C GIUEN THE IEMFEFIATURE T(K) ANTI TENSITY

C CALCULATES FRESSUFE F (MPA) ANII ITS IIEFIUUATUES W.F.T II AT

C CONSTANT T IIFIIIIMFA/(MOL/IIMZ)) ANI W.F.T T AT CONSTANT II

C [IFIIT (MFA/K), ENEFGY U ANI EN!THALFY H(J/MOL), ENTFOFY ANI

C SFECIFIC HEATS CF ANI CU(J/MOL.KI), VELOCITY OF SOUNI CS(M/S),

C ANI COMFFESSIEILITY $\operatorname{COMF(1/MFA).~}$

C IF THE ENIRY FOINT IS IN THE 2-FHASE FIEGION (IFHASE=2) IT

C. FETUFINS $C F=C O M F=C S^{\prime}=0$.

C IF IIIFINII=1 IT CALCULATES ONLY F ANI [IFII.

C ?. ON THE SATURATION CUFUE (ISAT.NE.O) :

C GIVEN THE TEMFEFATUFE T, IT CALCULATES THE IIENSITY [I, LATENT HEAT

C ANL ALL THE ABOUE FROFERTIES ON THE VAFOF SIIIE (ISAT=-1), OR THE

C LIRUIE SILIE (ISAT=1).

IMFLICIT IIOUELE FFECISION $(A-H, O-Z)$

COMMON/THEFIA/IFHASE, LI' III, IIF'LIT, U, H, ENTKOF', CF, CU, CS, COMF

COMMON/SATUR/ISAT, HEAT, IULIQ, DUAF

COMMON/COEFS/A $(20), Q(20)$

COMMON/CFITS/TC, XMLWGT, FHOC, F'C, F'CON, UCON, SCON, IIFCON

IIIMENSION $S(2), X K(2), S[I(2)$

EQUIVALENCE (F.W1, A(5)), (F.W2, A(4)), (FW3,A(2)),

$1(A M C, A(13)),(A M 1, A(14)),(A M 2, A(15)),(A M 3, A(16))$,

$(F \cdot 00, Q(11)),(F \cdot 20, Q(12)),(F 40, Q(13))$,

$(F \circ 1, Q(18)),(F \cdot 21, Q(19)),(F 41, Q(20))$,

$(A A, A(10)),(X K O), A(7)),(X K 1, A(12)),(F W 11, Q(9))$,

(ALFHA,Q(10)), (ALHT,Q(15)), (BESR,A(9))

C

DATA XMLWGT/28.0541L10/

$\mathrm{XK}(1)=\mathrm{XKO}$

$X K(I)=X K 1$

$T E E=(T-T C) / T C$

$T W=-T C / T$

IIT $W=1$. $[10+T W$,

IF (ISAT, NE. O) GO TO 10

F.HC) $=[1 / F$ :HOC:

CALL CONUEF ( $F$ H , TEE, AMU, TH1, FI , FHO1, S, FHOII, EFIF )

GO TO 12

$10 \quad$ TH $1=1 \cdot[10$

IF (ISAT,$E R \cdot-1)$ TH $1=-0.99999999999999999[10$

IF (ISAT,ER. 1) TH1 $=0.99999999999999999110$

$R 1=[1 T W /(1$, DO-EESQ $)$

CALI. SS (F:1, TH1, S, SII)

$F H O=T H 1 *\left(X K O * F 1 * * A(6)+X K 1 * F^{\prime} 1 * * R(16)\right)+A(1) *(S(1)+S(2))$

$\mathrm{F} H \mathrm{HO}=1$. IIO+FW11*[TW+K:HO

II $=$ KIHO*FIHOC

AMU $=0.110$

$12 T$ T $1=$ TH $1 *$ TH1

$T T 2=T T 1 * T T 1$

F'WO $=1 \cdot\left[10+\left[1 T W *\left(F W 1+\left[1 T W *\left(F \cdot W 2+\left[1 T W * F^{\prime} W 3\right)\right)\right.\right.\right.\right.$

FWMU $=A M U * F \cdot H O L I$

F'OTH=F'OO+F'2O*TT $1+F \cdot 40 * T^{2} 2$

$F 1 T H=F 01+F 21 * T T 1+F 41 * T T 2$

[IF'WO $=X K$ O $* F^{\prime} O T H * F: 1 * *(2,-A L F \cdot H A)$

LFW $1=X K 11 * F 1$ TH*Fi $1 * *(2,-A L H I)$

$\Pi F W=A A *([I F)+[I F \cdot W 1)$

$F W=F^{\prime} W O+F^{\prime} W M U+I I F ' W$

IF (I IIF INII.EQ.1) GO TO 11

【IF'O[IT $=F^{\prime} W 1+\left[1 T w *\left(2,\left[10 * F^{\prime} W 2+3, n 0 * F^{\prime} w 3 *[1 T W)\right.\right.\right.$

IMOLT $=A M 1+[1 T W *(2,[10 * A M 2+3$, DOO*AM3*[ITW $)$

$U W=$ IIFO IIT - F'HO LMOLI $+F W 11 * A M U+S(1)+S(2)$

$H W=F W-T W * U W$

$A M W=A M U+A M C+[T W *(A M 1+[1 T W *(A M 2+[1 T W * A M 3))$

C 
IF'HASE $=1$

CALL AUX(F.1, TH1, II2FIIT2, IIZFIIMT, DIFELIM2, AA, XK, SII, CUCOEX)

IIFIIII $=$ IIFCON*II*T/IISF.IMT2

IF ( IIIFINI.EQ.1) GO TO 15

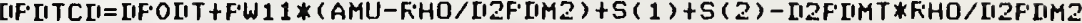
IIF'WITW $W=F \cdot W-T W *$ IIF'IITCE

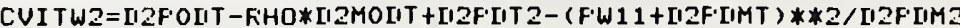

CUW=CUITW2*TW*TW

$C F \cdot W=C U W+$ II2F'IIMZ*IIF'WIITW*IIF'WLITW/ (FHO*FHO )

COMF' $=1$, $[10 /$ ( II*IIF'IIII)

CS $=1$. II3*IISDF T (CF'W/CUW*IIF IIII) / ISSRF'T ( XMLWGT)

IF (ISAT,EQ.0) GO TO 14

FHO $1=1$. $[10+F \cdot W 11 *[1 T W+A(1) *(S(1)+S(2))$

RHO2 $=X K O * F 1 * * A(6)+X K 1 * R 1 * * R(16)$

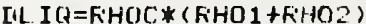

IIUAF $=$ FiHOC $*($ KHO $1-$ RHO2 $)$

IIF'IIT 2=F'W-TW* (UW+F'HO*IIMOIIT)

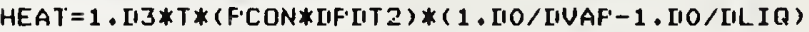

GO TO 14

13

IF'HASE $=$ ?

IIF. IIII $=0$.

IF ( IIIFINI.EQ.1) GO TO 15

IIF'IITCI $=U W+$ F'HO* IMMOITT

IIF'WI'T W=F'W-TW*IIF'IITCII

CUIO $=(2,-A L F \cdot H A) *(1,-A L F \cdot H A) * F^{\prime} 1 * *\left(-A L F^{\prime} H A\right) * X N O * F^{\prime} O T H$

CUI $1=(2,-A L H I) *(1,-A L H I) * F 1 * *(-A L H I) * X K 1 * F 1 T H$

CUI TW2 $=[12 F O[I T-R H O * I 2 M O I I T+A A *(1, /(1,-$ EESR $)) * * 2 *(C V I O+C U I 1)$ CUW $=$ CUITW2*TW*TW.

$C F \cdot W=0$.

LOMF $=0$.

$\mathrm{CS}=0$.

C

[IF' IIT $=$ F. CON*IIF'WIITW

$S C O N I=S C O N / I$

$\mathrm{U}=\mathrm{UW} * \mathrm{UCON} / \mathrm{II}$

$H=H W * S C O N I * T$

ENTR'OF:=SW*SCONII

CU $=$ CUW*SCONII

$C F=C F W * S C O N I$

$F \cdot=F \cdot W * F C O N * T$

FE TUFN

ENI 
SUBROUT INE CONUER ( $R H O$, TEE, AMU, TH1, K1 , FHO1S, S1, RHOLI, ERROR 1)

C THIS ROUTINE TRAANSFORMS TEMPERATURE ANII IIENSITY TO THE

C FARAMETRIC VAFIAELES $R$ AND THETA ACCORIING TO THE REVISED

C ANI EXTENUED SCALING EQUATIONS.

IMFLICIT REAL $* 8(A-H, O-Z)$

COMMON /COEFS/ $A(20), Q(20)$

IIMENSION $S 1(2), S I(2)$

EQUIVALENCE (RETA, $A(6)),(\operatorname{IELTA}, A(11)),(X K 1, A(12)),(C C, A(1))$

1 , (ALHI, Q(15)), (ALPHA, Q(10)), (BESQ, A(9)), (F11, Q(9))

2 , (IIELI, Q(14)), (F1W, Q(18)), (P2W,Q(19)), (F4W, Q(20))

3. $(A A, A(10)),(X K O, A(7)),(S 00, A(17)),(S 20, A(18)),(B E T A I, Q(16))$

TSTAR = TEE+1. T10

DTSTIN $=1$. IIO $-(1$. DO/TSTAR $)$

RI $1=$ ITSTIN

IF (IITSTIN.LT, 0,) RI=DTSTIN/(1, -BESQ)

$\mathrm{TH} 1=0$.

IF (DTSTIN.LT.O.) TH1=1 .

CALL SS(R1,TH1, S1, SD)

FHOII $=1$. DO + P11*IITSTIN

RHOIIT $=$ FHODI + CC*S1(1) + CC*S1(2)

[IRHO = RHO - RHODIT

$A M U=0.110$

IF (DTSTIN.GT . O. IO) GO TO 1

RHO1CO $=$ XKOO*R1**EETA + XK1*R1**BETAI

TWOFAZ = RHO1CD

IF (DAES (DFHO).GT.TWOFAZ) GO TO 1

FHO1S = DSIGN (FH01CO, UKHO $)+$ CC*S1(1)

$\mathrm{TH} 1=\operatorname{IISIGN}(1, \operatorname{IIO}, \mathrm{DRHO})$

ERROR1 $=1.110$

GD TO 999

1 CONTINUE

IF (IIRHO.NE.O.DO) GO TO 2

$\mathrm{TH} 1=0 . \mathrm{DO}$

$\mathrm{R} 1=\mathrm{DTSTIN}$

FiHO1S $=\operatorname{CC} * S 1(1)$

2 CONTINUE

C RULE FOF' FIRST F'ASS

$Y 1=$ IITSTIN

DIENI = RHO - RHOIIT

CALL RTHETA(F'1, TH1, LEN1, Y1)

$T T=T H 1 * T H 1$

AMU = AA* R1**(EETA*IIELTA)*TH1*(1, I0-TT)

$Y 1=$ ITSTIN + CC*AMU

CALL SS(FI,TH1,S1,SD)

RHOWEG $=\times K 1 *($ RI $* *$ EETAI $) * T H 1+C C * S 1(2)$

FHO1S $=$ DEN $1+C C * S 1(1)+$ RHOWEG

ERROR1 = FHO - FHODI - RHO1S

IF ( [IAES (ERFOR'1).LT.1.[I-5) GO TO 999

C FULE FOR SECOND F'ASS

IIEN12 = RHO - FHODI - CC*S1(1) + RHOWEG

IF (DEN12.EQ. IIEN1) [IEN12= IIEN1 - 1. [I-06

CALL FITHETA(R1, TH1, IIEN12,Y1)

$T T=T H 1 * T H 1$

AMU = AA* $K 1 * *($ BETA*IELTA $) * T H 1 *(1,[10-T T)$

$Y 1=$ IITSTIN + CC*AMU

CALL SS(F1,TH1,S1,SD)

FHOWEG $=$ XK1*F1**BETAI*TH1 + CC*S1(2)

FHO1S2 = IIEN12 + CC*S1(1) + FHOWEG

EFROF2 = RHO - FHOLI - RHO1S2

IF ( IIAES(EF'ROR2).GT.1.I1-5） GO TO 998

ERROR1 = EFFOF'2

RHO1S $=$ FHO1S2

GO TO 999 
YYU LUNTINUL

C RULE FOR NTH F'ASS

IIEN2 = [LEN12

IO 44 ISIG $=1,10$

SLOFE = (EF'OR'2-EF'R'OF'1)/(IIEN2 - IEN1)

HOLD = UEN2

DEN2 = UEN1 - (EF'F'OF'1/SLOPE)

CALL FTHETA (R'1, TH1, IIEN2,Y1)

$T T=T H 1 * T H 1$

AMU $=A A * R 1 * *($ BETA*UELTA $) * T H 1 *(1, D 0-T T)$

$Y_{1}=$ IITSTIN + CC *AMU

CALL SSLF1,TH1,S1, SII)

FHOWEG $=$ XK'1*R1**EETAI*TH1 + CC*S1 (2)

FHO1S $=$ IIEN2 + CC*S1(1) + RHOWEG

EFIOFI 1 = EFIROR2

EF'FIR'2 = F'HO - F'HODI - F'HO1S

IF ( IIAES (EF'F'OF'2) .LT,1.[1-6) GO TO 999

DEN1 = HOLII

44 CONT INUE

IF (IIABS (EFFIOF'2) , GT, 1,II-6) WRITE $(6,66)$ EFROR2, RHO, DTSTIN, DEN2, CC

66 FOKMAT $(1 X, '$ 'CONUER IIOES NOT CONUER'GE',5E12.6)

999 CONTINUE

RETUR'N

ENI

EOF AT LINE 87

*

BLOCKLITA

TIOURLE F'RECISION A,Q

COMMON /COEFS/A (20), Q (20), JAA (20), ANAMES (20), BNAMES (20)

C THIS SUBFOUTINE SUFFLIES THE F'AFAMETERS USEII IN THE EQUATION

C OF STATE.

IJATA $A /-.007811[10,0 .[10,0,[10,-18.1475110,5.3350110, .325110,1,12289$

$100,0,110,1.3757110,19,3214110,4,8200, .54617110,-36,4889110,-27.395534110$

$2,-12,209110,-11.880200,4 * 0,50 /$

DIATA $Q /-.008369110,-.00121810,0.010,282,3452110,7.634110,5.0403110$

$1, .113090110,-.777110,12 * 0.10 /$

DATA ANAMES/GHC, 6 HCOT3

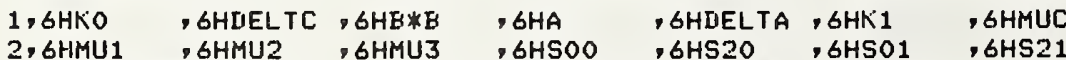

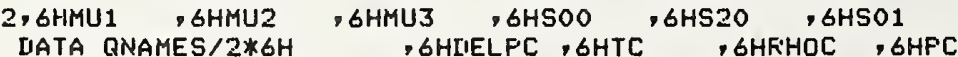

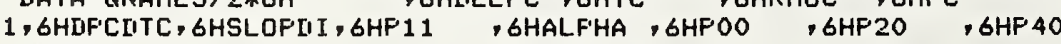

2,6HDELTAI, 6HALF'HAI, 6HEETA I, 6HGAMMAI, 6HF'01, 6HP21, , 6HF'41

END

EOF AT LINE 17

* 
SUEROUTINE CONGEN

C THIS SUBROUTINE CALCULATES ALL QUANTITIES NOT UEPENEIENT ON R OR THETA IMPLICIT IIOUELE PFECISION (A-H,O-Z)

COMMON /COEFS/ A(20), Q (20)

IIATA IIELF', IJELPM, IIELF'H, DELFMH/1.0-6,-1, D-6, 1, 00000100, 99999900 EQUIVALENCE (IIELI, $Q(14)),(D F H O C, A(3)),($ EETA,A $(6)),(D E L T A, A(11))$

1, (COTEE2, $A(4)),($ IIFIIT, A(5)), (BESQ,A(9))

$2,(C C, A(1)),(A A, A(10)),(T C K, Q(4)),(F H O C, Q(5)),(P C, Q(6)),(X K O, A(7))$

3, (ALHI, Q(15)), (EETI, Q(16)), (GAMI, Q(17)), (PO1,Q(18)), (P21, Q(19))

$4,(A L F H A, Q(10)),(P 00, Q(11)),(P 20, Q(12)),(P 40, Q(13)),(P 41, Q(20))$

$5,(500, A(17)),(520, A(18)),(501, A(19)),(521, A(20))$

ALFHA $=2.00-A(6) *(A(11)+1.00)$

GAMMA = EETA*(IIELTA - 1. IIO)

DELI $=0.5000$

$A L H I=A L F H A$ - IIELI

BETI = EETA + DELI

GAMI = GAMMA - UELI

ERR = 2. DO*EETA*UELTA - 1. DO

FOO = (HETA*(DELTA-3.DO)-BESQ*ALFHA*GAMMA)/2.DO*

1 BESQ*EESQ*(2, IIO-ALF'HA)*(1, IIO-ALF'HA)*ALF'HA)

F'20 $=-($ EETA* $($ DELTA-3, )-BESQ*ALFHA*ERR $) /(2, * E E S Q *(1,-A L F H A) *(A L F H A))$

F'40 $=(E F F-2) /$,2 . /ALFHA

$500=(2,-A L P H A) * P O O$

S20 = -BETA*(DELTA-3.)/2./BESQ/ALFHA

$U A=Q(1)$

$[\mathrm{B}=\mathrm{Q}(2)$

$R A=\amalg A /(1, D 10-E E S Q)$

$\mathrm{RB}=[\mathrm{IE} /(1, \mathrm{DO}-\mathrm{EESQ})$

SWO $=500+520$

DF $0=5 W 0 *(R A * *(1,-A L F H A)-R B * *(1,-A L P H A))$

F01 = (BETA*(IIELTA-3,[10)-3, I10*IIELI-A(9)*ALHI*(GAMI)

$1 /(2, \operatorname{DO} * A(9) * A(9) *(2$, DO-ALHI $) *(1$, D1O-ALHI $) * A L H I)$

F.21 $=-(($ EETA*(IIELTA-3, D10 $)-3$, DO*UELI I A (9)*ALHI*ERR )

$1(2.10 * A(9) *(1,110-A L H I) * A L H I))$

F.41 $=\left(.5 * E F^{\prime} F\right.$ - 1.) /ALHI

SO1 $=(2,-A L H I) * F 01$

S21 $=-($ EETA*IELTA-3.*EETI $) / 2 . /$ BESQ/ALHI

FTW $=P 01+F 21+P 41$

SW1 $=501+S 21$

$D R 1=S W 1 *(R A * *(1,-A L H I)-F E * *(1,-A L H I))$

C

GO TO 10

ENTRY QQGEN

10 IF (XKO.LE.0. DO ) XKO $=0.000100$

CC $\quad Q(6)=A(13)$

CC $\quad Q(8)=A(14)$

D $1=C C * A A * \times K O * D R O$

II $2=C C * A A * A(12) *[1 / 1$

$Q(9)=(Q(B) *(1 . /(1,-[B)-1 . /(1,-D A))+D 1+D 2) /(D B-D A)$

$Q(7)=(A(5)+1,10) * Q(6) / Q(4)$

$Q(3)=A(8) * Q(7)$

FETURN

END

EOF AT LINE 53

* 
SUEROUTINE AUX (F1, TH1, LIFDT2, D2FEIMT, D2FLIM2, AA, XK, SL, CUCOEX)

C THIS ROUTINE CALCULATES SECOND IJERIVATIUES OF THE SCALED

C EQUATION OF STATE

IMF'LICIT FEAL*B ( $A-H, O-Z)$

COMMON /COEFS/ $A(20), Q(20)$

IIIMENSION XK(2),S(2), SI (2),W(2),Y(2),Z(2),COEX(2)

EQUIUALENCE (CC,A(1)), (BETA,A(6)), (BESG,A(9)), (DELTA,A(11))

1, (ALFHA, $Q(10)),(S 00, A(17)),(S 20, A(18)),(S 01, A(19)),(S 21, A(20))$

DELI $=0.010$

$S(1)=S 00+520 * T H 1 * T H 1$

$S(2)=S 01+521 * T H 1 * T H 1$

$\operatorname{Sn}(1)=2, * T H 1 * S 20$

$\operatorname{SD}(2)=2 * * T H 1 * S 21$

$W W=0.510$

$Y Y=0 . \mathrm{DO}$

$Z Z=0 . D O$

GAMMA = BETA* (DELTA - 1.DO)

$T T 1=T H 1 * T H 1$

TER = 2. DO*BETA*IELTA - 1. INO

$G=(1,+(B E S G * T E R-3) * T T$,1 - EESQ*(TER-2.)*TT1*TT1)

CUCOEX $=0.10$

DO $30 I=1,2$

ALHI = ALFHA - IIELI

BETI = $\mathrm{BETA}+$ IELI

GAMI = GAMMA - IDELI

$W(I)=(1,-A L H I) *(1,-3, * T T 1) * S(I)-\operatorname{BETA} * \operatorname{IELTA} *(1,-T T 1) * T H 1 * S \mathrm{~L}(\mathrm{I})$

$W(I)=(W(I) *(F 1 * *(-A L H I))) / G$

$W(I)=W(I) * \times K(I)$

$W W=W W+W(I)$

$Y(I)=$ EETI*(1,DI0-3, INO*TT1)*TH1 - BETA*DELTA*(1, IIO-TT1)*TH1

$Y(I)=(Y(I) *(R 1 * *(B E T I-1 . D 0))) * X K(I) / G$

$Y Y=Y Y+Y(I)$

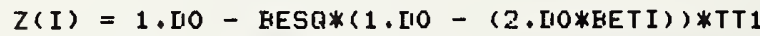

$Z(I)=(Z(I) *(F 1 * *(-G A M I))) * \times K(I) / G$

$Z Z=Z Z+Z(I)$

$A 1=($ BETA* (IIELTA-3, IO) -3, IO*LELI-EES( $* A L H I * G A M I)$

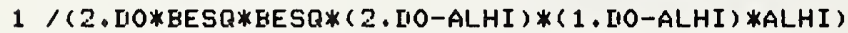

$A 2=1+(($ BETA* $($ IELLTA-3. IO $)-3$. IO $*$ IIELI-EESQ*ALHI*TER $)$,

1 (2. DO*BESQ*(1, DO-ALHI)*ALHI ))

$A 2=-A 2$

$A 4=1$, DOO+( $(T E F-2,[10) /(2$, DO *ALHI $))$

$F 1=A 1+A 2+A 4$

$\operatorname{COEX}(I)=((2, \mathrm{DO}-\mathrm{ALHI}) *(1, \mathrm{DO}-\mathrm{ALHI}) *(\mathrm{R} 1 * *(-\mathrm{ALHI})) * \mathrm{~F} 1 * X K(I))$

CUCOEX $=$ CUCOEX $+\operatorname{COEX}(I)$

30

LIELI $=0.5 \mathrm{DO}$

CONTINUE

IIFET2 = AA*WW

II2F.UMT $=Y Y+A A * C C * W W$

I2FUM2 $=Z Z / A A+2 \cdot D O * C C * Y Y+(C C * * 2) * A A * W W$

RETURN

ENI

EOF AT LINE 51 
C GIVEN THE TEMFEFATURE T(K), FRESSURE F(MPA), ANII AN INITIAL

C GUESS IIENSITY IIGUESS $(K G / M 3)$, THIS ROUTINE FINUS THE

C COFRESFONIIING IIENSITY II(KG/M3) IN THE FANGE (IMMIN, IIMAX).

C IF T IS BELOW THE CRITICAL ISOTHERM, IIGUESS MUST BE SET

C EQUAL TO THE CFITICAL [IENSITY, OTHERWISE IT MAY YIELI

C EFFIONEOUS FESULTS. IF THE ENTRY FOINT IS IN THE 2-FHASE

C REGION CFITICAL IIENSITY IS F'ETURNEI FOF' $D$.

c

IMFLICIT F'EAL $* 8(A-H, O-Z)$

COMMON/THERIM/IF'HASE, [IF' [III

[I= [IGUESS

IIO $10 \mathrm{I}=1,20$

CALL THEFIMO $\left(1, T, \mathrm{II}_{1}, \mathrm{~F}^{\prime} \mathrm{F}^{\prime}, \mathrm{FF}^{\prime}, \mathrm{THETA}\right)$

$P D I F=P F-F$

IF (IF'HASE,EQ, 2) GO TO 18

[IELII=-F[IIF/IIF'III]

$[I=[I+[I E L I I$

IF (II.LT . DIMIN) $\quad \mathrm{J}=\operatorname{LIMIN}$

IF (II, GT . IIMAX) II=IIMAX

IF ( IIAES ([IELII/L) , LT , 1, II-6) GO TO 19

GO TO 10

18 IF (IIAES (FIIIF) , LE , O, [10) GO TO 19

IF (FIIIF. LT , O+ DIO) II IIMAX

IF (FIIIF, GT , O, DIO) $\quad[1=[1 M I N$

10 CONTINUE

WRITE $(6,21)$ T,F,PF, D

21 FOFIMAT ( $5 X$, 'DIF INI IIOES NOT CONUEFGE':', $4 F 12.4$ )

19 RETURN

ENII

EOF AT LINE 32

SUEROUTINE TFINII (F',T)

IMFLICIT DOUELE PFECISION ( $A-H, O-Z$ )

COMMON/THEFM/ IFHASE, IIF III, IIFUT,U,H, ENTROF', CF , CU, CS, COMF COMMON/SATUF/ I UAF, HEAT, FHOL, FHHOG

COMMON/COEFS/A(20), Q (20)

$c$

COMMON/CRITS/TC, XMLWGT, RHOC, FC, FCON, UCON, SCON, IIFCON

$\mathrm{T}=\mathrm{TC}-1 \cdot \mathrm{L} / \mathrm{O}$

II=FiHOC

ISAUE $=$ IVAF

IVAF' $=2$

IIO $10 \quad \mathrm{I}=1,20$

CALL THEF'MO(O,T, [I, F'P, R1, TH1)

[IT $=\left(F^{\prime} F^{\prime}-F^{\prime}\right) /$ IIF [IT

$\mathrm{T}=\mathrm{T}-\mathrm{IIT}$

IF (T.GT.TC) GO TO 12

IF ( IIAES (IIT/T) ,LT.1.D-8) GO TO 20

GO TO 10

$12 \quad \mathrm{~T}=\mathrm{TC}-0.001510$

10 CONTINUE

WFITE $(6,11)$ F', F'P, T

11 FOFMAT $(1 X / 1 X$ 'TFIND IIOES NOT CONUERGE :',3F $12,6 /)$

20 IUAF $=$ ISAVE

FEETURN

EN[! 
C MAIN FROGFAM TO GENEFIATE TAELES IN IITFFEFENT UNITS IMPLICIT FEEL $* 8(A-H, O-Z)$

FEEAL ANAMES, QNAMES

C

COMMON /COEFS/ A(20), Q(20), JAA(20), ANAMES (20), QNAMES(20)

COMMON /UNITS, IT, III,IF, IH,NT,NI, NF, NH, FT, FI, FF, FH

COMMON /CFITS/ TC, XMLWGT, FHHOC, FC, FCON, LCON, SCON, IIFCON

COMMON /THEFM/ IFHASE, DIFII, IFIT, U,H,ENTEOF, CF, CU, CS, COMF

C

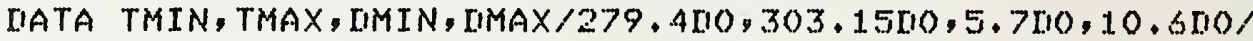
IIATA XMLWGT/23.0541DO/

C

CALL CONGEN

c

$r C=Q(4)+A(B)$

$\mathrm{FH} H O C=Q(5)+A(3)$

$\mathrm{F} C=Q(6)+Q(3)$

$P C O N=F C / T C$

UCON $=1,[13 * F F$

$S C O N=U C O N / T C$

IIFCON $=$ FCON / FHOC / FHOC

C

CALL, UNIT

C

1 WFITE $(6,101)$

101 FOFMATS' OENTEF DENSITY (MOL. LIMZ)' ')

FEAI $(5,100$, ENII=999) [IFNSIN

100 FOFIMAT ( )

UENS = DENS INKFI

C

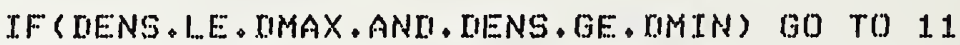
WFITE $(6,102)$

102 FOFMATS" 1'EQUATION' 'S VALITIITY**')'

C GO TO 1

C

11 IDF INI $=0$

WFI TE $(6,130)$

130 FOFMAT (' ENTEF STAFT TEMF, , STOF TEMF,, \& INCFEMENT') REAI (5, 100) TT1, TT2, TTTNC

$\mathrm{T} 1=\mathrm{TTT}(\mathrm{TT} 1)$

$\mathrm{T} 2=\mathrm{TTT}(\mathrm{TT} 2)$

IF (T1.LT, TMIN ) TI = TMIN

IF (T2.GT. TMAX) T2=TMAX

$\operatorname{TT} 1=\operatorname{Tr} T(T 1)$

$\operatorname{TT} 2=\operatorname{TTI}\left(T_{2}\right)$

C

WFITE $(6,110)$ IIENSIN

110 FORMAT $\left(1, y(1), T_{20}, 15.2,{ }^{\prime}\right.$ TSOCHOFE', 1 )

WRTTE $(6,111)$

111 FOFMATS' ', T2, 'TEMFEFATUFE', T15, 'FFESSURE', T25, 'ISOTHEFM', $1 T 35$, ISOCHOFE', T45, 'INTEFNAL. , T55, 'ENTHALFY', T65, 'ENTFOFY', 2T76, 'CW', TS6, 'CF', T94, 'SOUNO')

WFI I TE $(6,113)$

* 


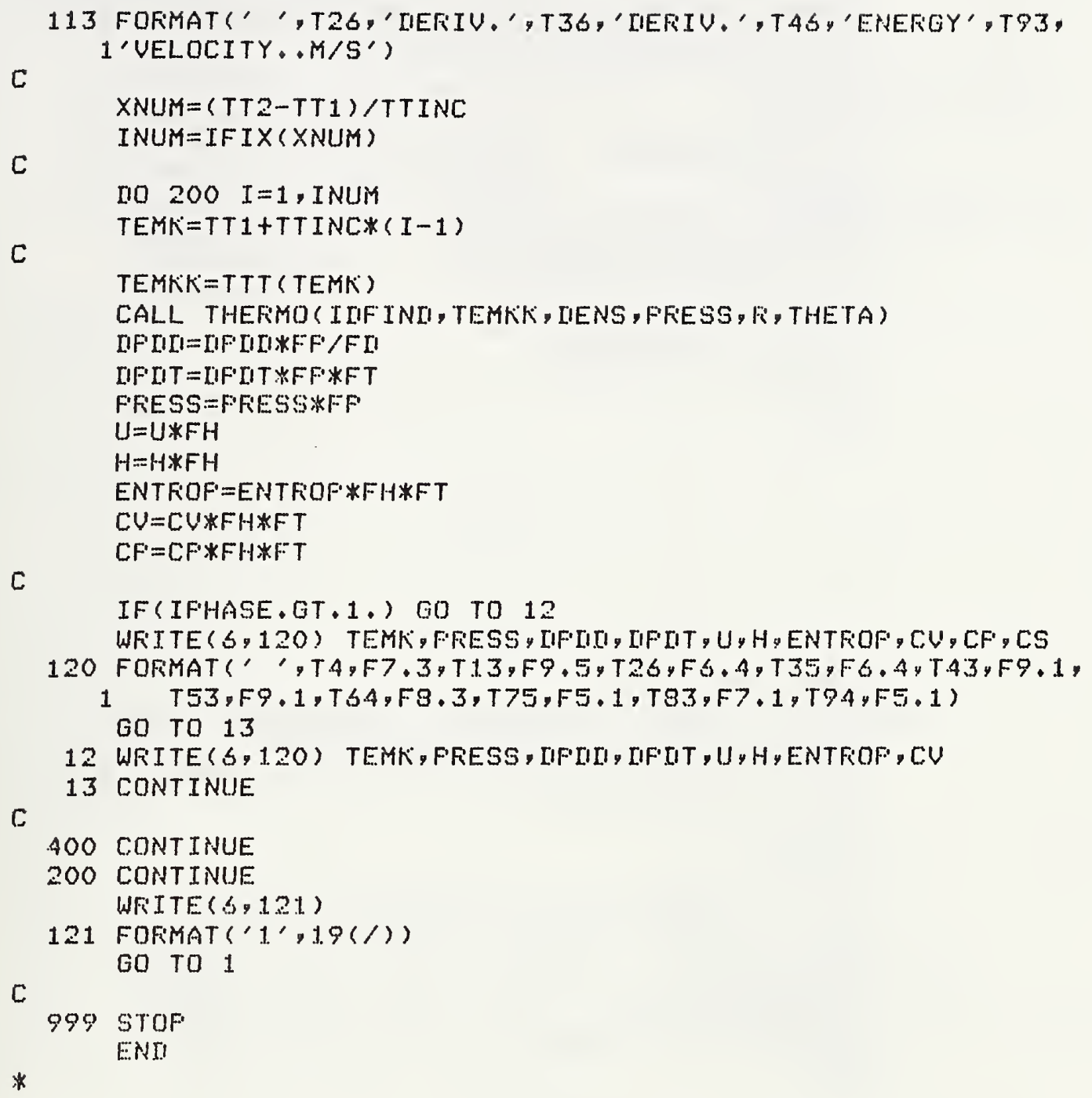

C

IF (IFHASE,GT.1.) GO TO 12

WFITE(S, 120) TEMK, FFESS, IFIII, IFIIT, U,H, ENTFOF, CU, CF, CS

120 FOFMAT(' ', TA,F7.3,T13,F9,5,T26,FG.4,T35,F6.4, T.43,F9,1, 
FUNCTTON TTT(T)

C FUNCTION TO CONUERT INFUT TEMFEFATUFES IN EXTEFNAL UNITS TO IEE $K$ IIOUBLE FFECISION T, TTT, FT, FII,FF, FH

COMMON /UNITS/ IT,II,IF,IH,NT,NI,NF,NH,FT,FI,FF,FH GO TO $(1,2,3,4): I T$

$1 \quad \mathrm{TTT}=\mathrm{T}$

$F T=1,1.10$

FIETUFIN

$2 \quad \mathrm{TTT}=\mathrm{T}+273.15 \mathrm{TH}$

$F T=1,[10$

FIETUFN

$3 \mathrm{TTT}=\mathrm{T} / 1,8 \mathrm{NO}$

$F T=.5555555555556110$

FETUFiN

$4 T T T=(T+459.67110) / 1.800)$

$F T=.555555555555650$

FETUFN

ENII

FUNCTION TTI (T)

C FUNCTION TO CONUEFT TNTEFNAL TEMFEFATURES IN LEG $K$ TO EXTEFNAL UNTT DOUBLE FFECISION T, TTI, FT, FI, FF" $F H$

COMMON , UNITS, IT, III,IF, IH,NT, NI, NF, NH, FT, FI, FF, FH

GO TO $(5,6,7,8)$, IT

$5 \mathrm{TTI}=\mathrm{T} * 1 . \mathrm{I10}$

FEETUFiN

S TTI $=\mathrm{T}-273.15 \mathrm{N10}$

$T T I=T T I * 1$, TIO

FETUFiN

$7 \quad \mathrm{TTI}=\mathrm{T} * 1,8 \mathrm{~N} \mathrm{O}$

FIETUFN

$8 T T I=T * 1.850-459.6720$

FETUFiN

ENI 


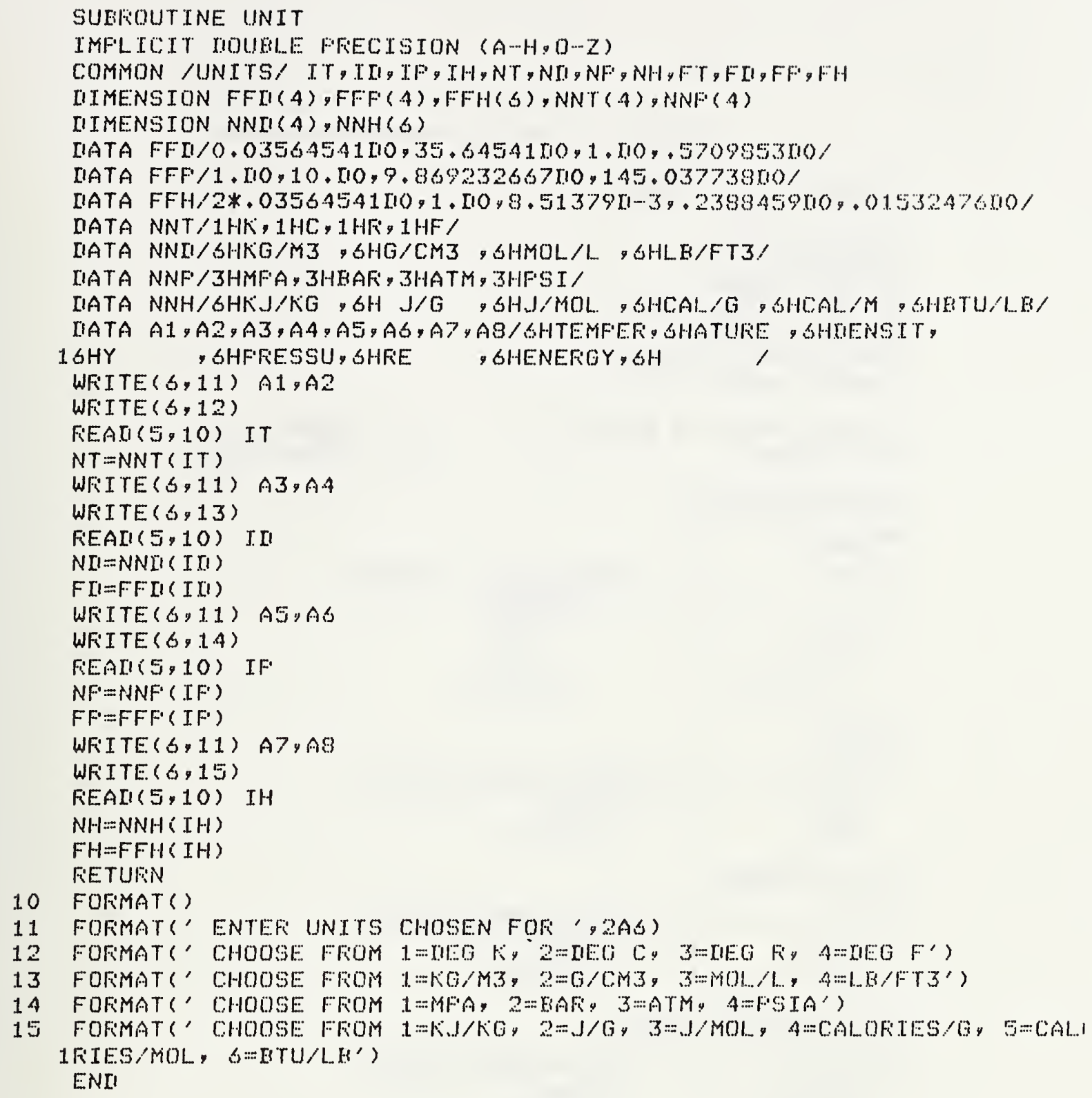


Units used in the Tables

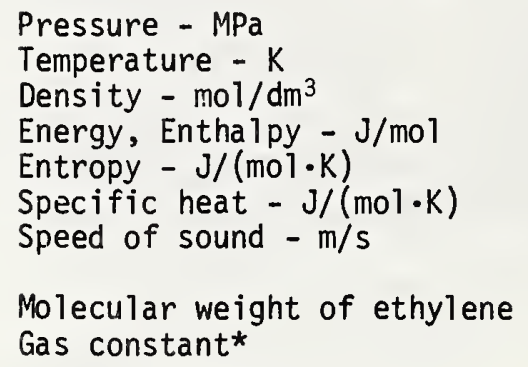

Reduction Factors

\begin{tabular}{l|c} 
Pressure & \\
\hline $\mathrm{MPa}$ to & Multiply table value by \\
\hline $\mathrm{Pa}$ & $10^{6}$ \\
bar & 10 \\
atmosphere & 9.869233 \\
$\mathrm{psi}, 1 \mathrm{bf} / \mathrm{in}^{2}$ & 145.037738
\end{tabular}

Density

\begin{tabular}{l|l}
$\mathrm{mol} / \mathrm{dm}^{3}$ to & Multiply table value by \\
\hline $\mathrm{kg} / \mathrm{m}^{3}$ & 28.0541 \\
$1 \mathrm{bm} / \mathrm{ft}^{3}$ & 1.7513585
\end{tabular}

Energy, Enthalpy

\begin{tabular}{l|l}
$\mathrm{J} / \mathrm{mol}$ to & Multiply table value by \\
\hline $\mathrm{kJ} / \mathrm{kg}$ & 0.0356454 \\
$\mathrm{BTU} / \mathrm{bm}$ & 0.01532476
\end{tabular}

Entropy, Specific heats

\begin{tabular}{c|c}
$\mathrm{J} /(\mathrm{mol} \cdot \mathrm{K})$ to & Multiply table value by \\
\hline $\mathrm{KJ} /(\mathrm{kg} . \mathrm{K})$ & 0.0356454 \\
$\mathrm{BTU} /(1 \mathrm{bm} \cdot \mathrm{R})$ & 0.00851375
\end{tabular}

Temperature

\begin{tabular}{l|l}
$K$ to & Transform table value by \\
\hline${ }^{\circ} \mathrm{C}$ & $\left(\mathrm{T},{ }^{\circ} \mathrm{C}\right)=(\mathrm{T}, \mathrm{K})-273.15$ \\
${ }^{\circ} \mathrm{F}$ & $\left(\mathrm{T},{ }^{\circ} \mathrm{F}\right)=\frac{9}{5}[(\mathrm{~T}, \mathrm{~K})-273.15]+32$ \\
$R$ & $(\mathrm{~T}, \mathrm{R})=\frac{9}{5}(\mathrm{~T}, \mathrm{~K})$
\end{tabular}

Speed of sound

\begin{tabular}{c|c}
$\mathrm{m} / \mathrm{s}$ to & Multiply table value by \\
\hline $\mathrm{ft} / \mathrm{s}$ & 3.28083
\end{tabular}

*The actual value of the gas constant is not required for the programs in this Tech. Note. The value given here is that used in reducing the original Burnett PVT data $[5,24]$ and in NBS Tech. Note $1045[7]$. 
FIGURES 


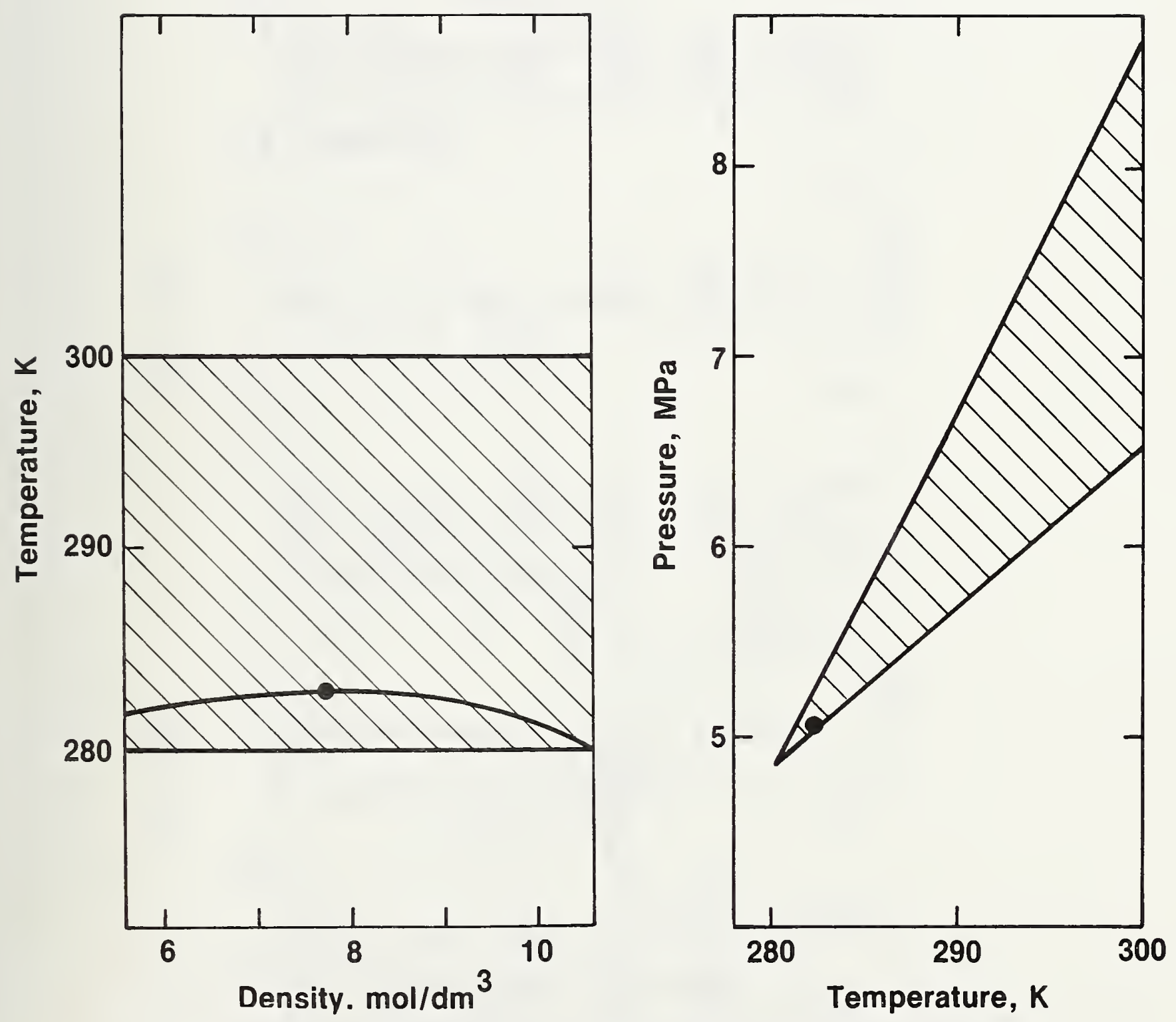

Fig. 1 Range of validity of the present formulation. Left: in temperature - density space. Right: in pressure - temperature space. 

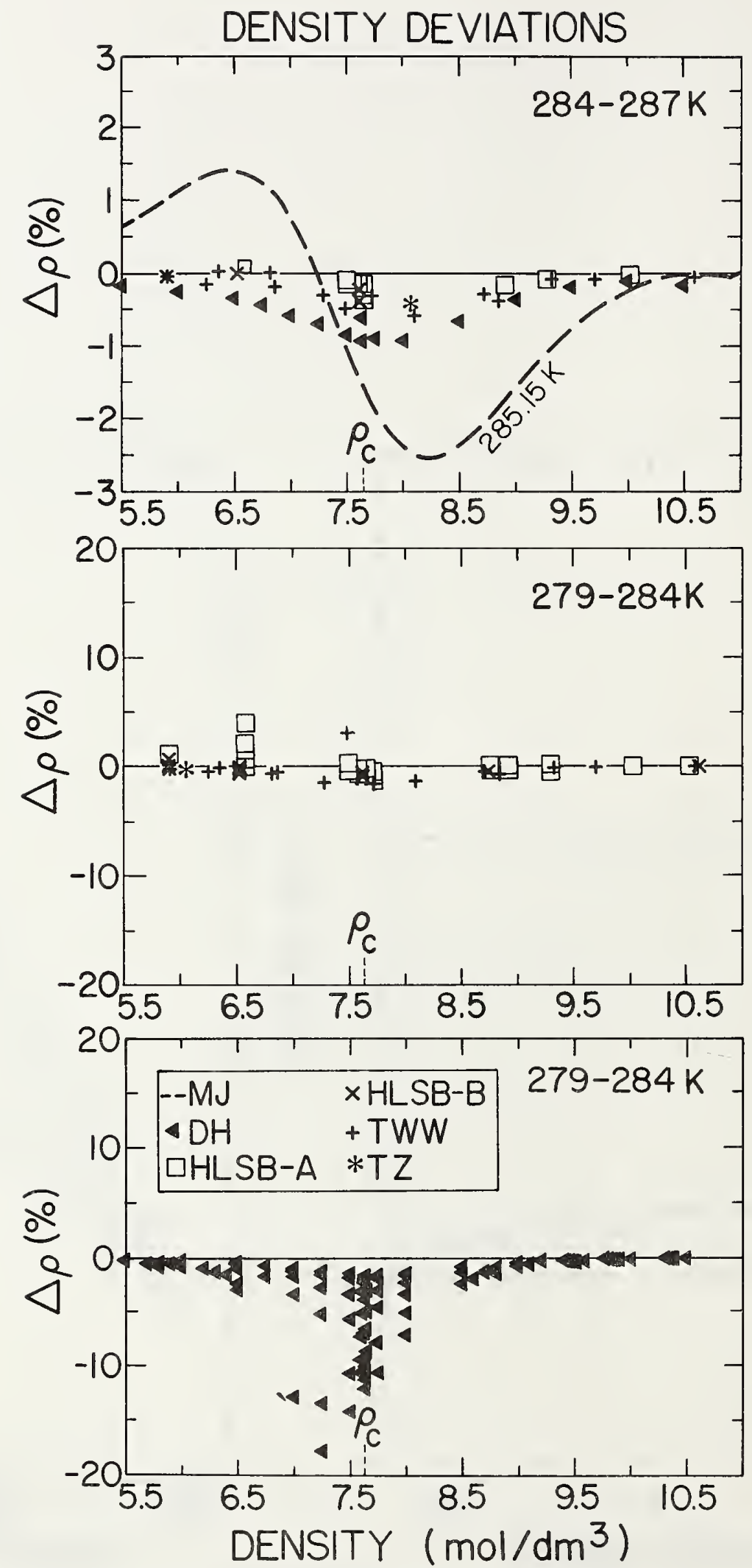

Fig. 2a Departures of the experimental densities of Douslin and Harrison ( $\bullet$ ), Hastings et al. ( $\square$, sample $A$ and $X$, sample B), Trappeniers et al. $(+)$ and Thomas and Zander ( $*$ ) from our formulation (baseline) if compared at the same temperature and pressure. The dashed curve represents the departures of the McCarty-Jacobsen formulation from our own. 

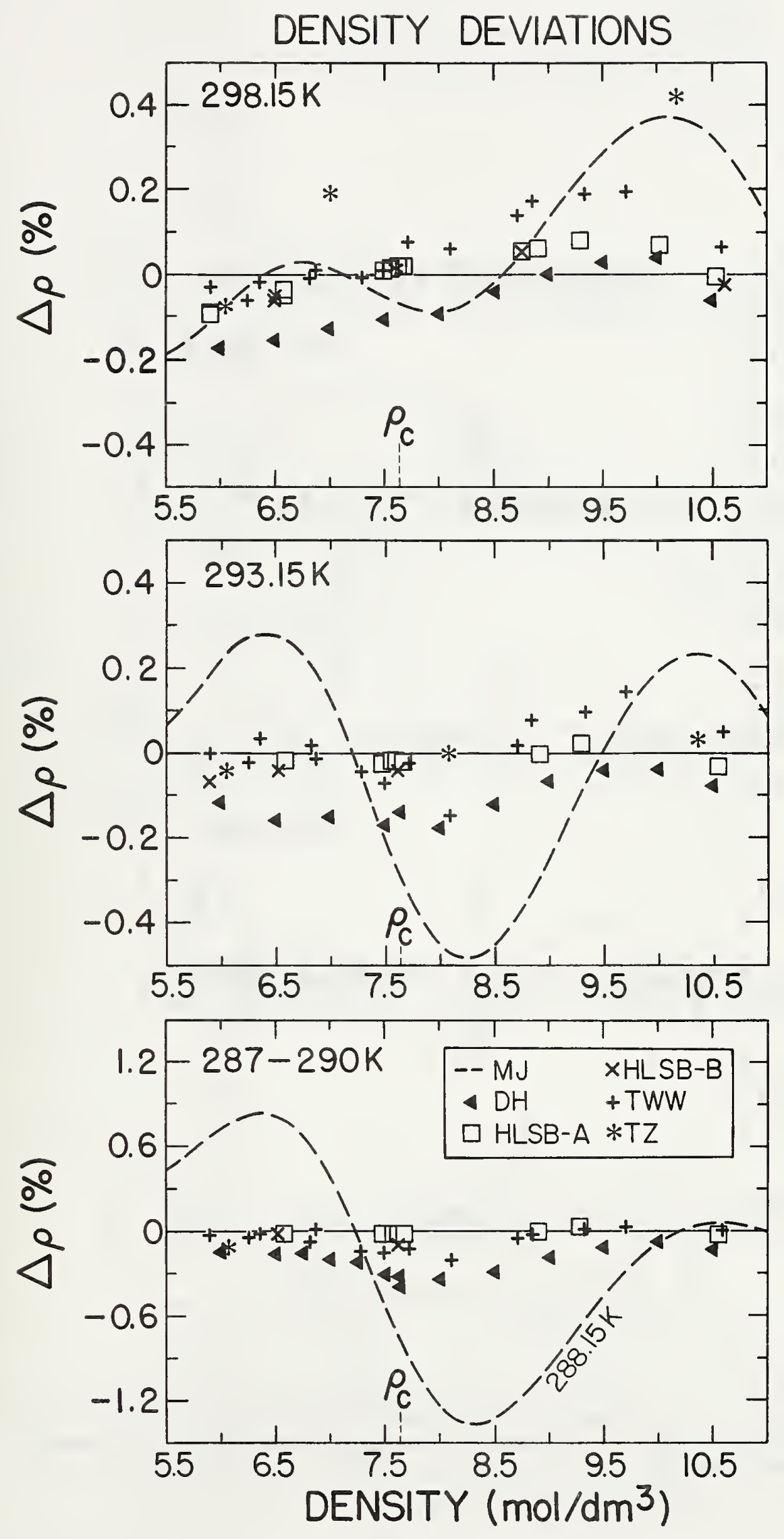

Fig. $2 b$ As in Fig. 2a.

77 


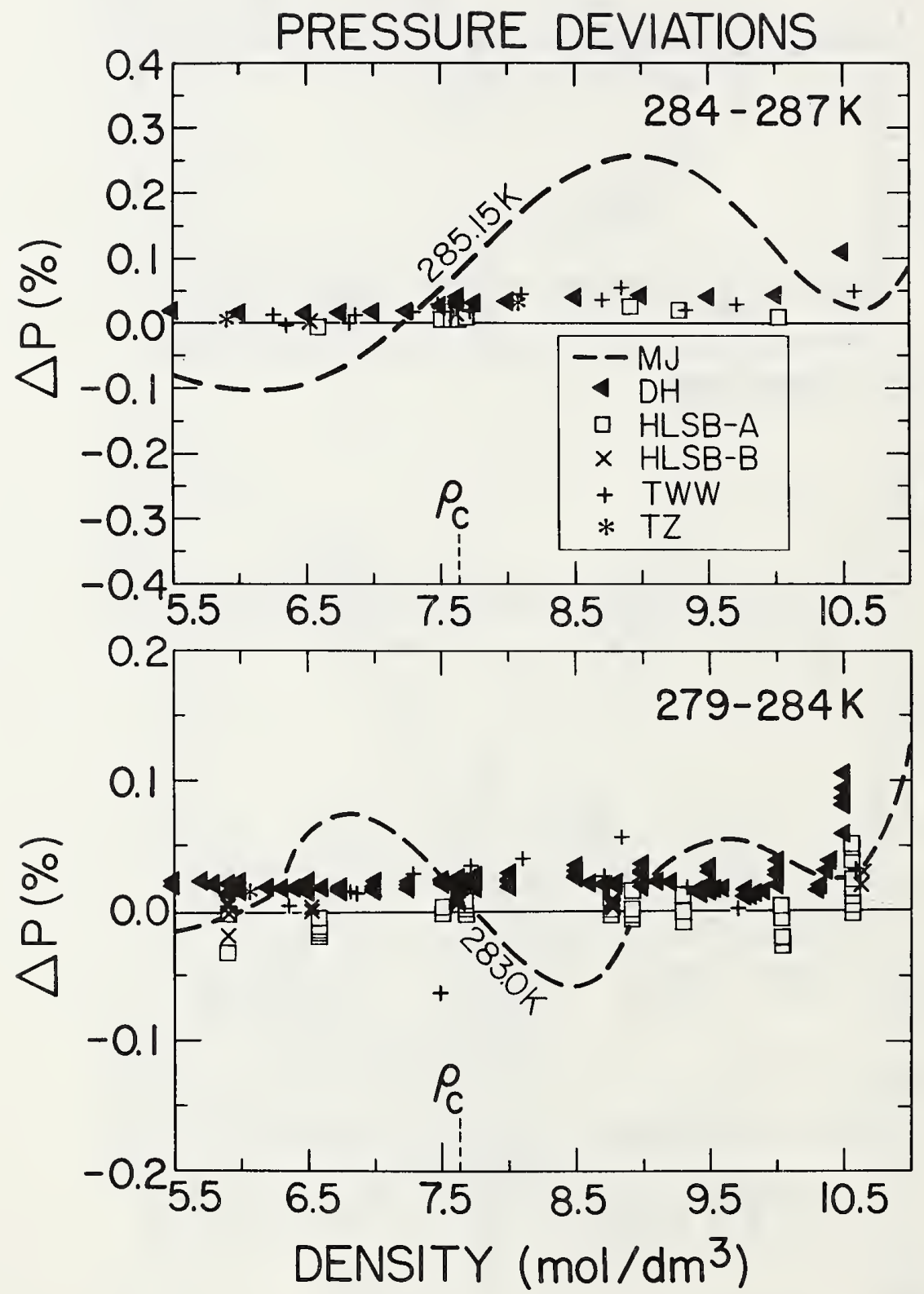

Fig. 3a Departures of the experimental pressures of Douslin and Harrison ( $)$, Hastings et al. ( $\square$, sample $A$ and $X$, sample B), Trappeniers et al. $(+)$ and Thomas and Zander $(*)$ from our formulation (base line) if compared at the same temperature and density. The dashed curve represents the departures of the McCarty-Jacobsen formulation from our own. 

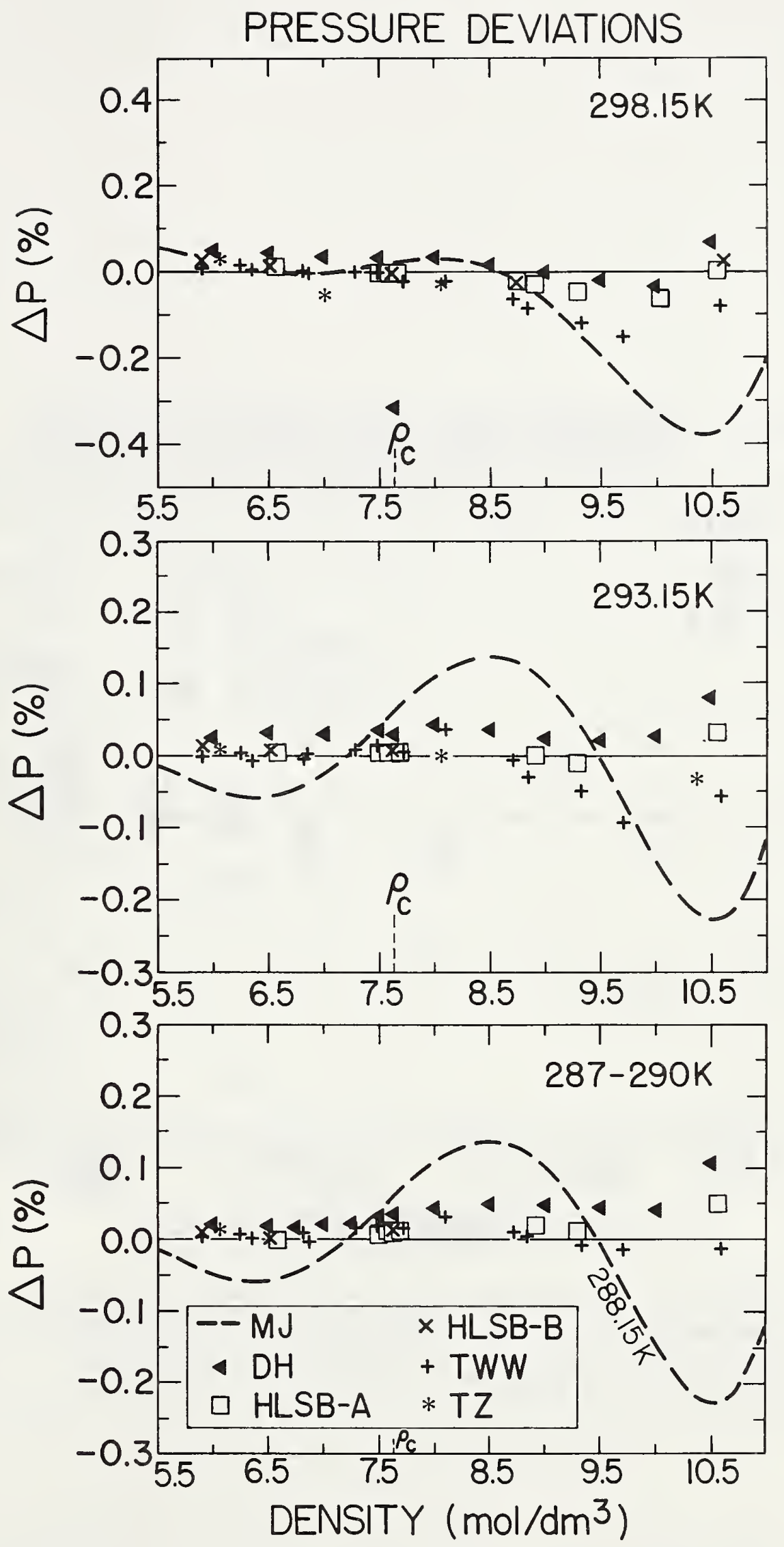

Fig. Bb As in Fig. Ja.

79 


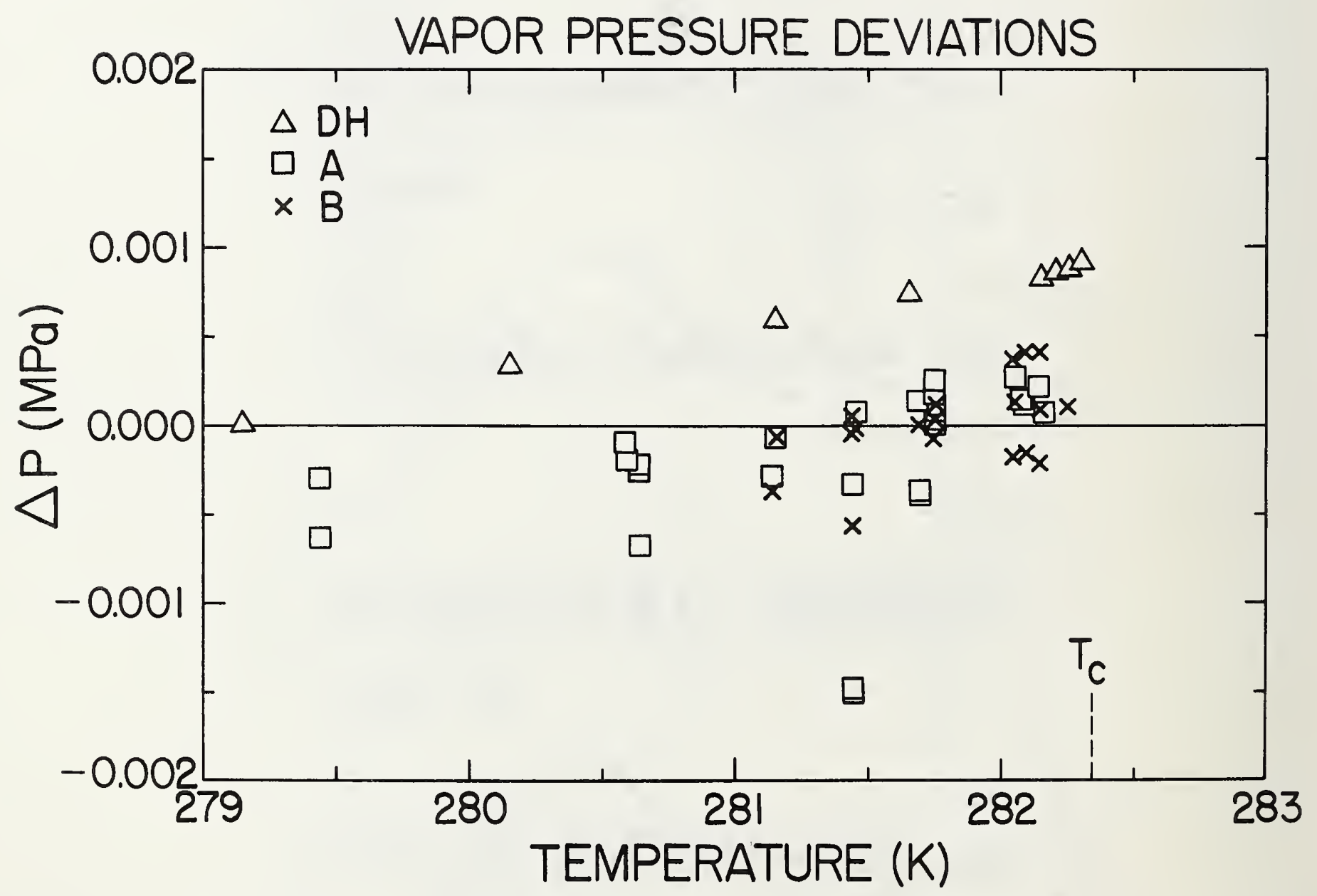

Fig. 4 Departures of the experimental vapor pressures of Dous] in and Harrison $(\Delta)$ and Hastings et al. ( $\square$, sample $A$ and $X$, sample $B$ ) from the present formulation. 


\section{DEVIATIONS OF COEXISTING DENSITIES}

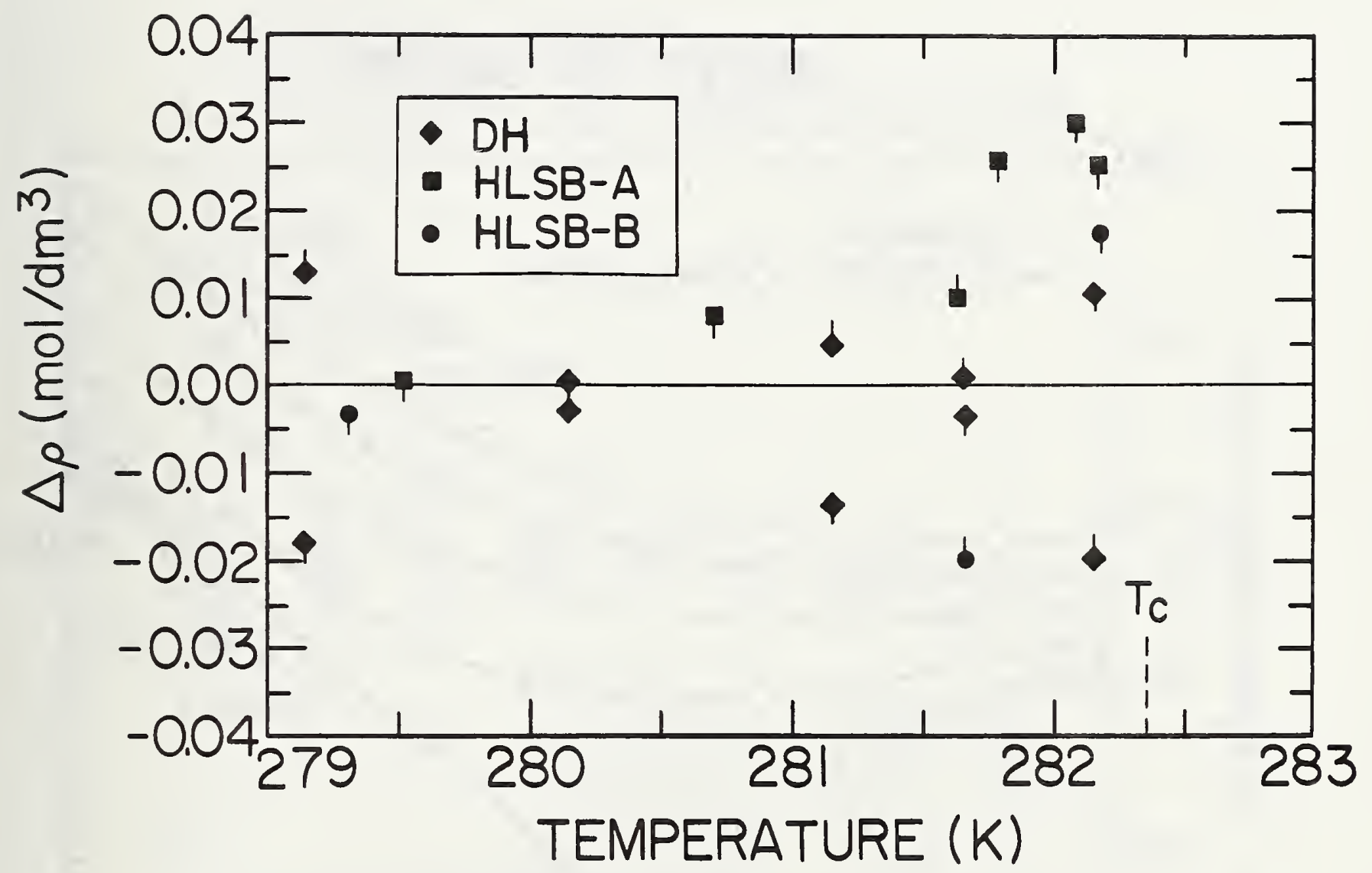

Fig. 5 Departures of experimental coexisting densities of Dous 7 in and Harrison ( $)$ and Hastings et al. ( from the present formulation. Upward-pointing line segments denote vapor densities; downward-pointing ones denote liquid densities. 


\section{SPEED OF SOUND}

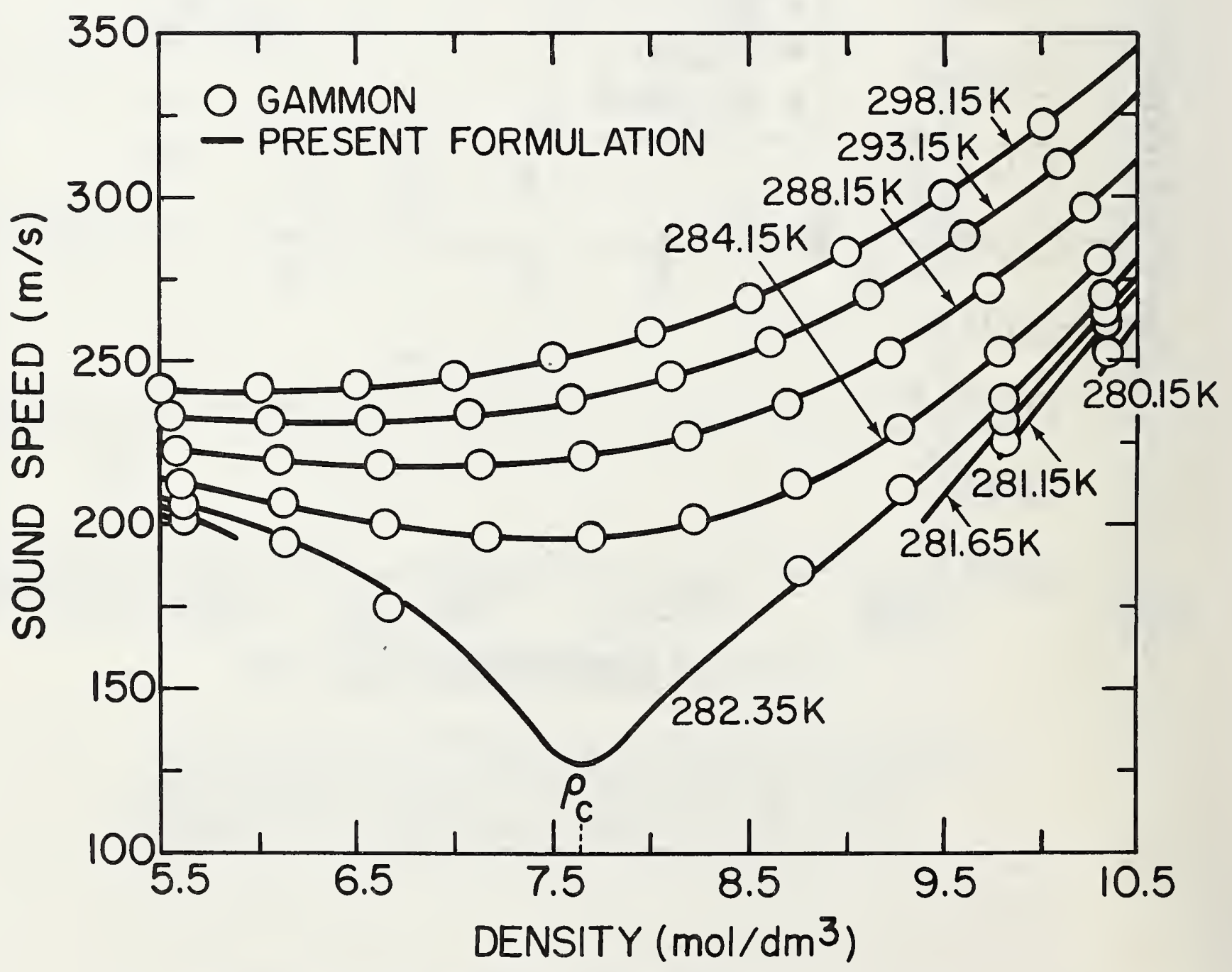

Fig. o The speed of sound as measured by Gammon compared with the present formulation. 


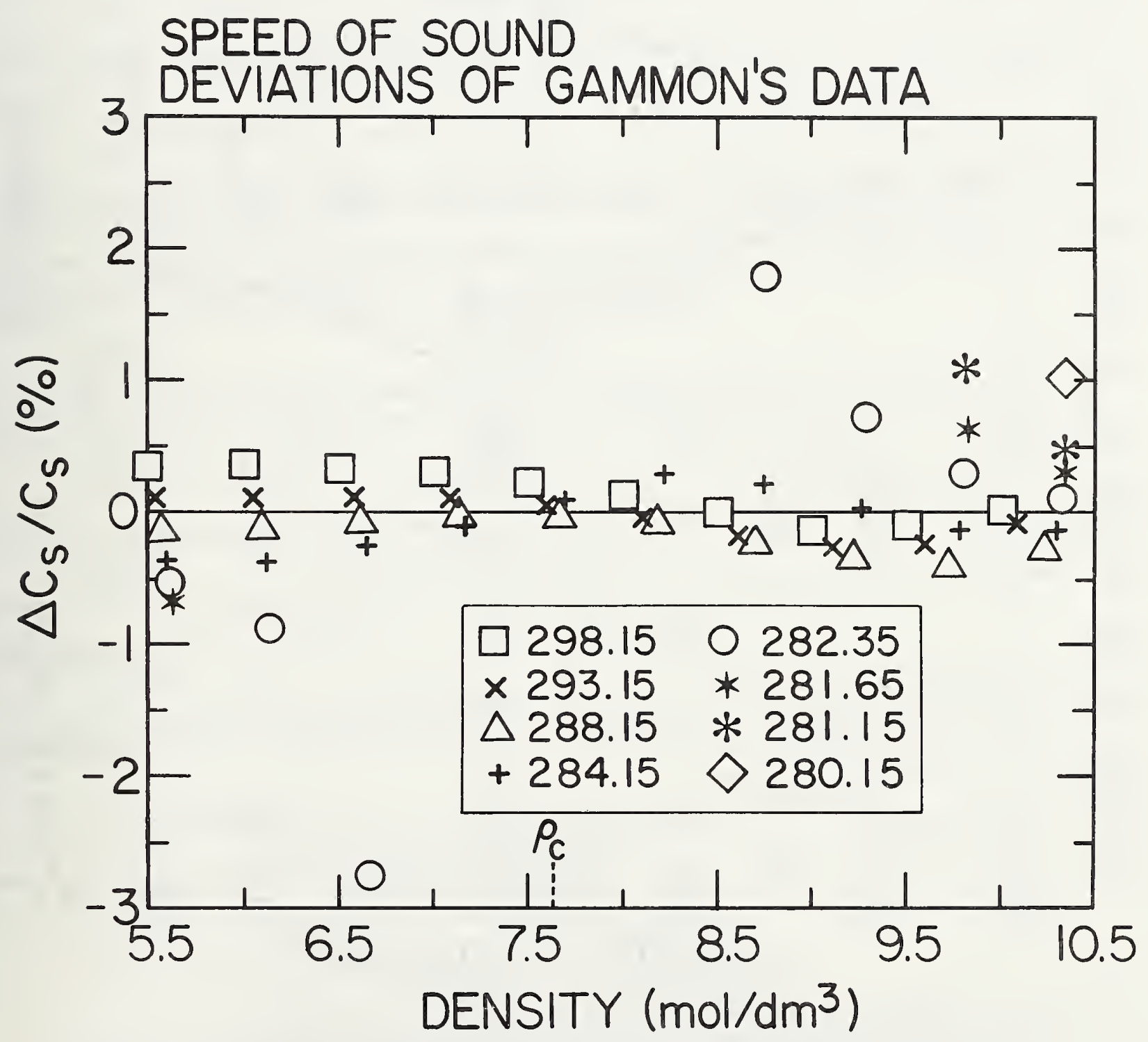

Fig. 7 Departures of Gammon's speed of sound data, in \%, from the present formulation. 

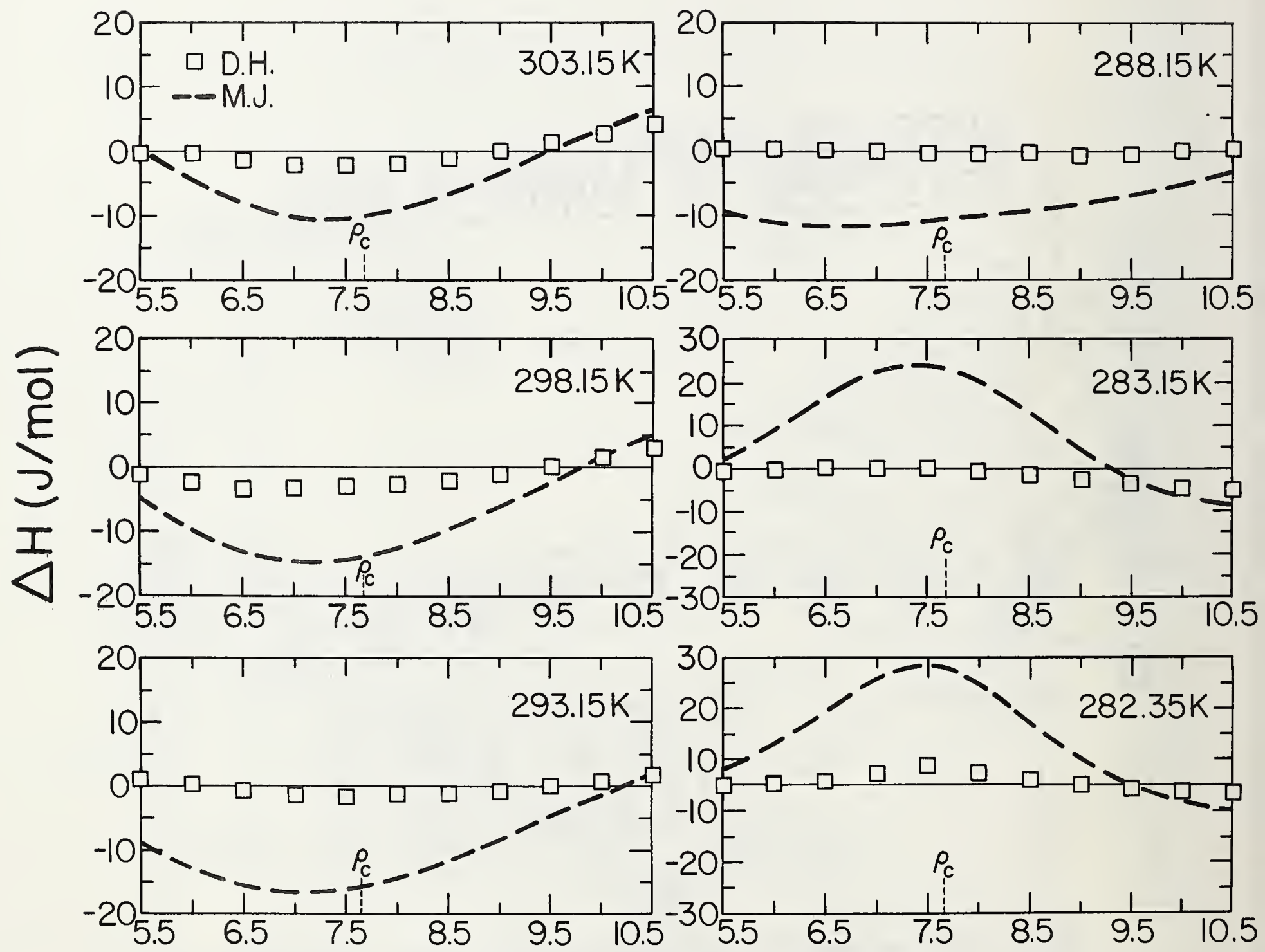

\section{DENSITY $\left(\mathrm{mol} / \mathrm{dm}^{3}\right)$}

Fig. 8 Comparison of the enthalpies of ethylene according to different formulations. The base line is the present formulation, which was anchored to the Dous 7 in and Harrison data at $7 \mathrm{~mol} / \mathrm{dm}^{3}, 288.15 \mathrm{~K}$. The squares are from the Douslin-Harrison calculation and the dashed curves from the McCarty-Jacobsen formulation. 

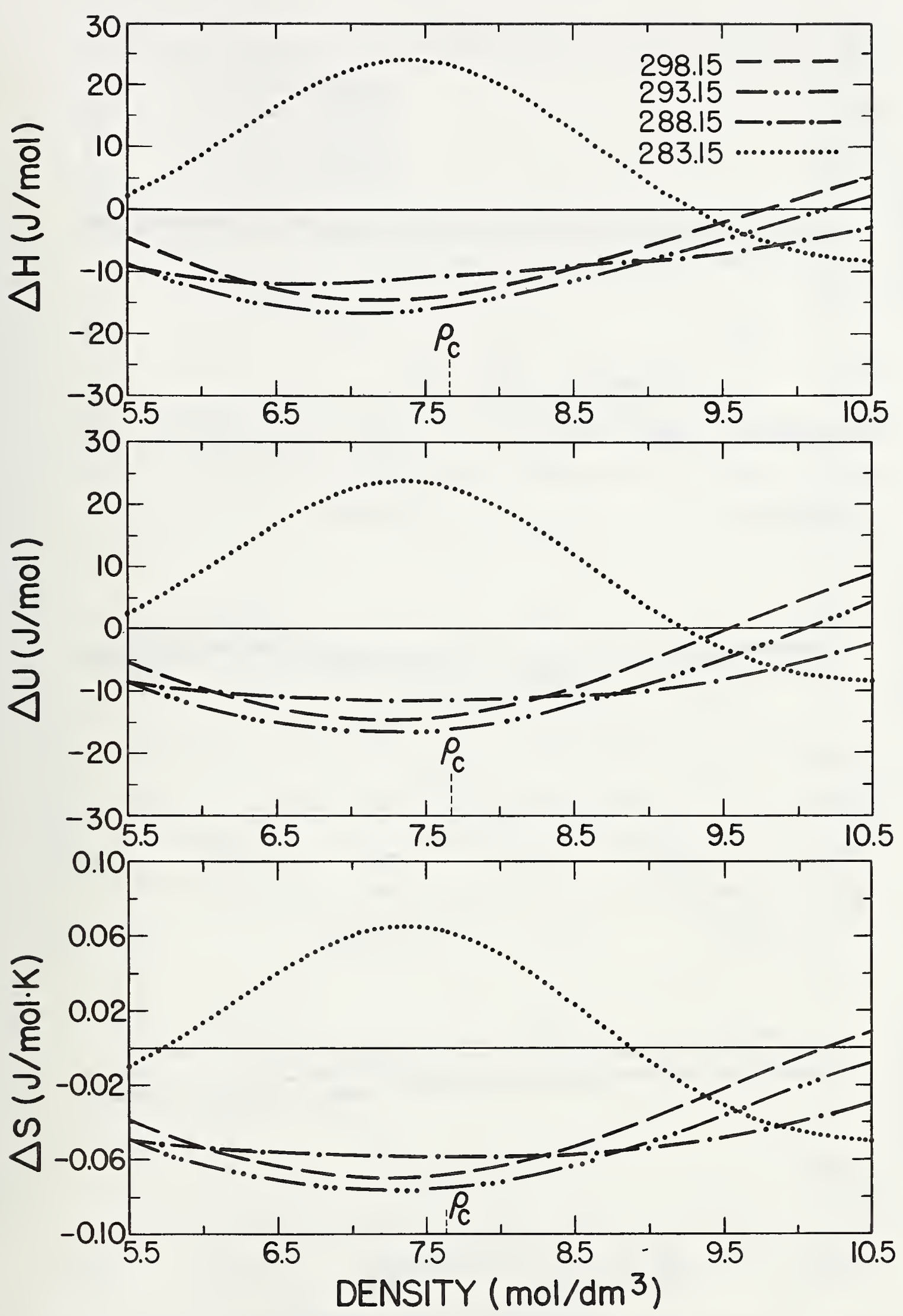

Fig. 9 The difference between the prediction of the McCarty and Jacobsen surface for several first-derivative thermodynamic properties and the present formulation. 

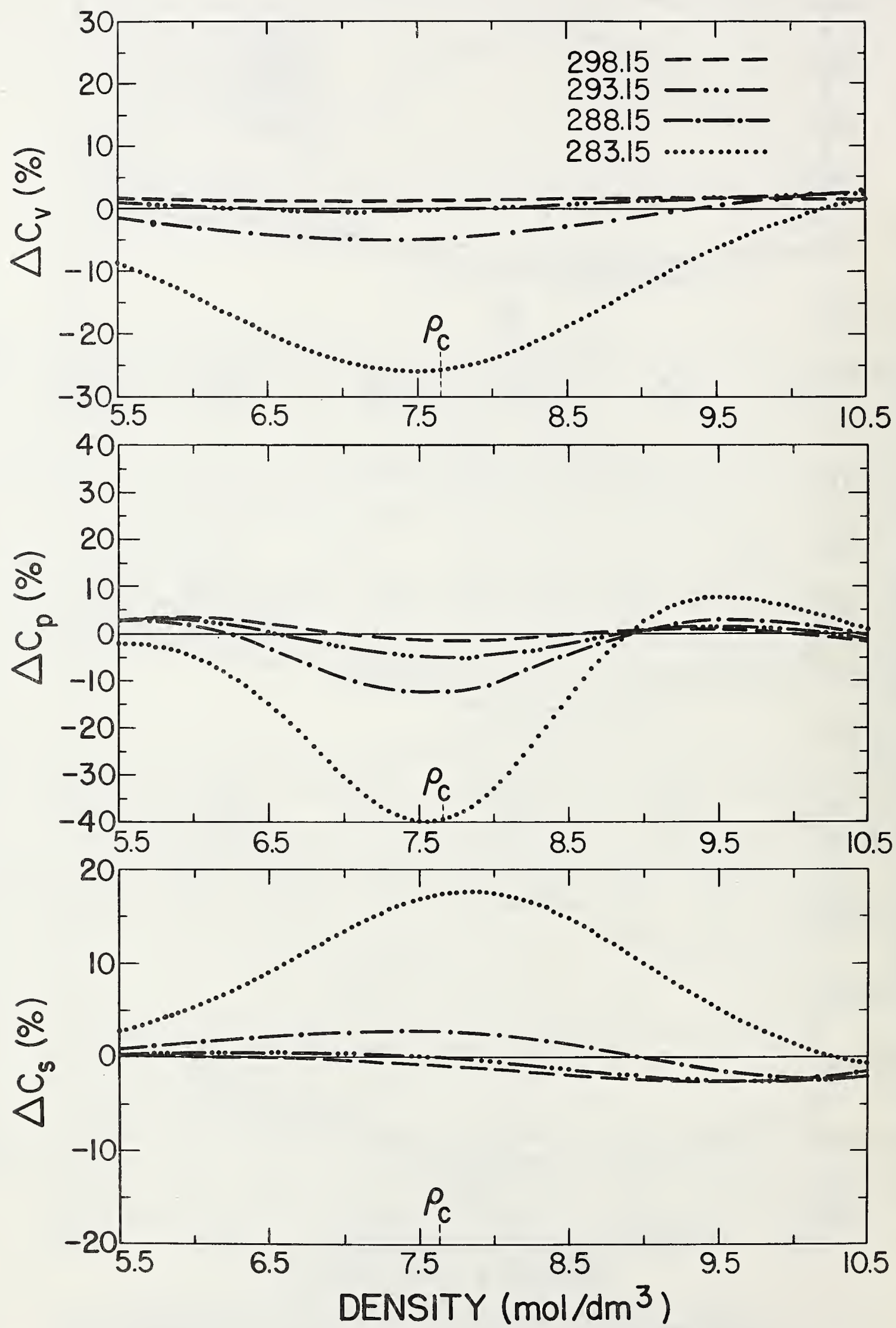

Fig. 10 Percentage differences between the McCarty-Jacobsen and the present formulation for second-derivative values. 


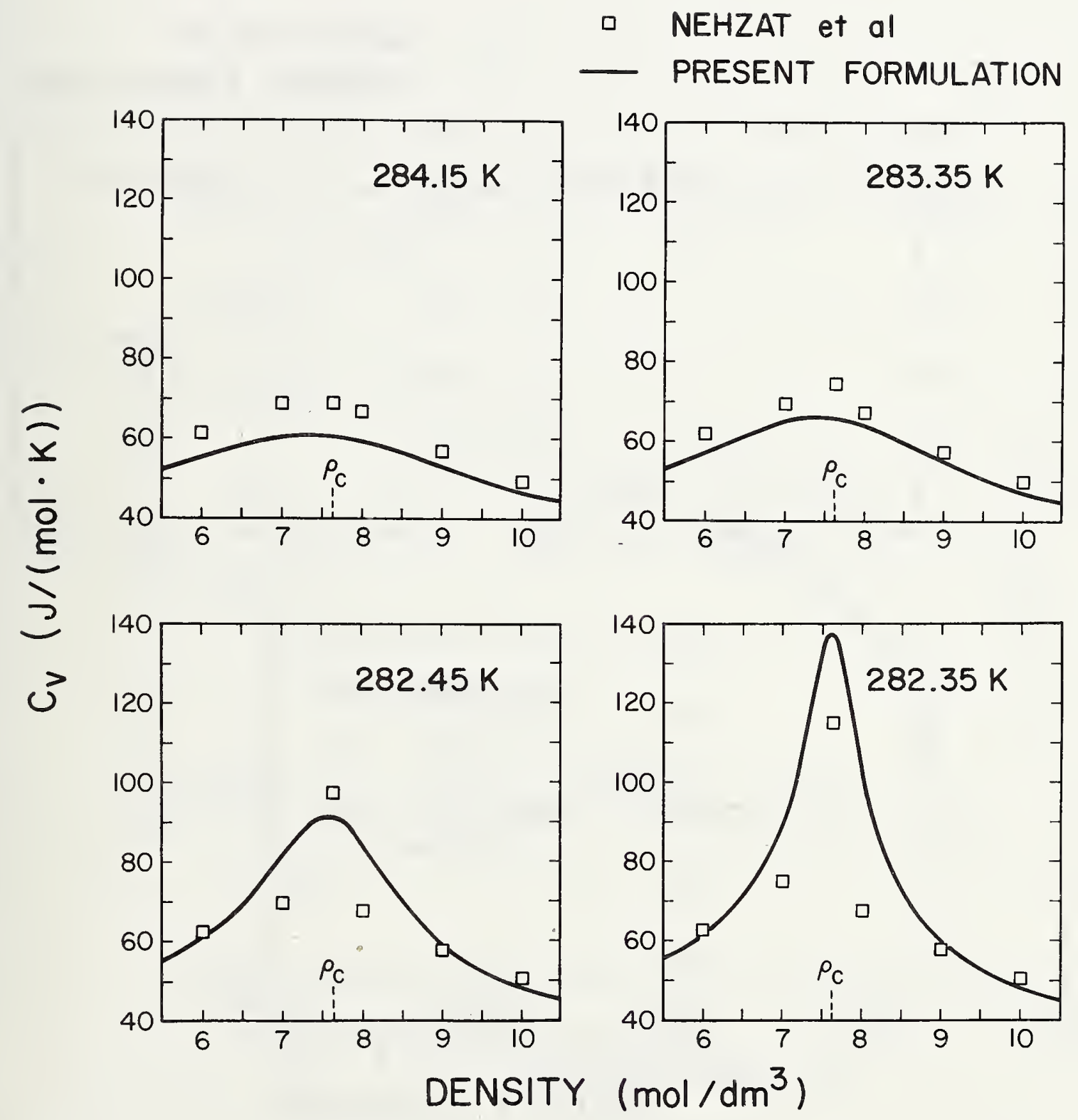

Fig. 11 Comparison of the specific heat $C_{y}$ predicted by Nehzat et al. [23] with our formulation in the one-phase region above the critical temperature. 


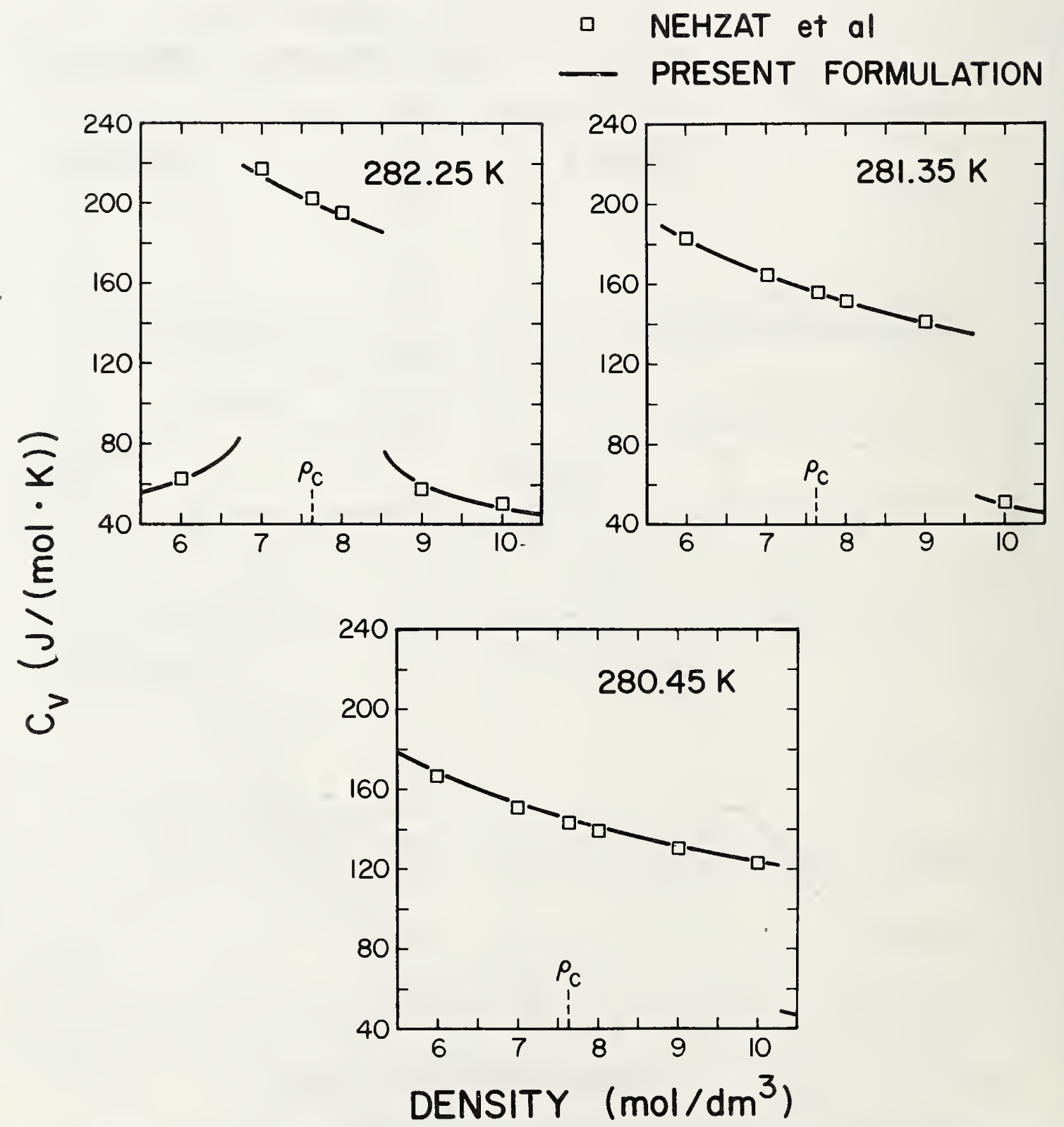

Fig. 12 Comparison of the specific heat $C_{y}$ predicted by Nehzat et al. [23] with our formulation in the two-phase region below the critical temperature. 


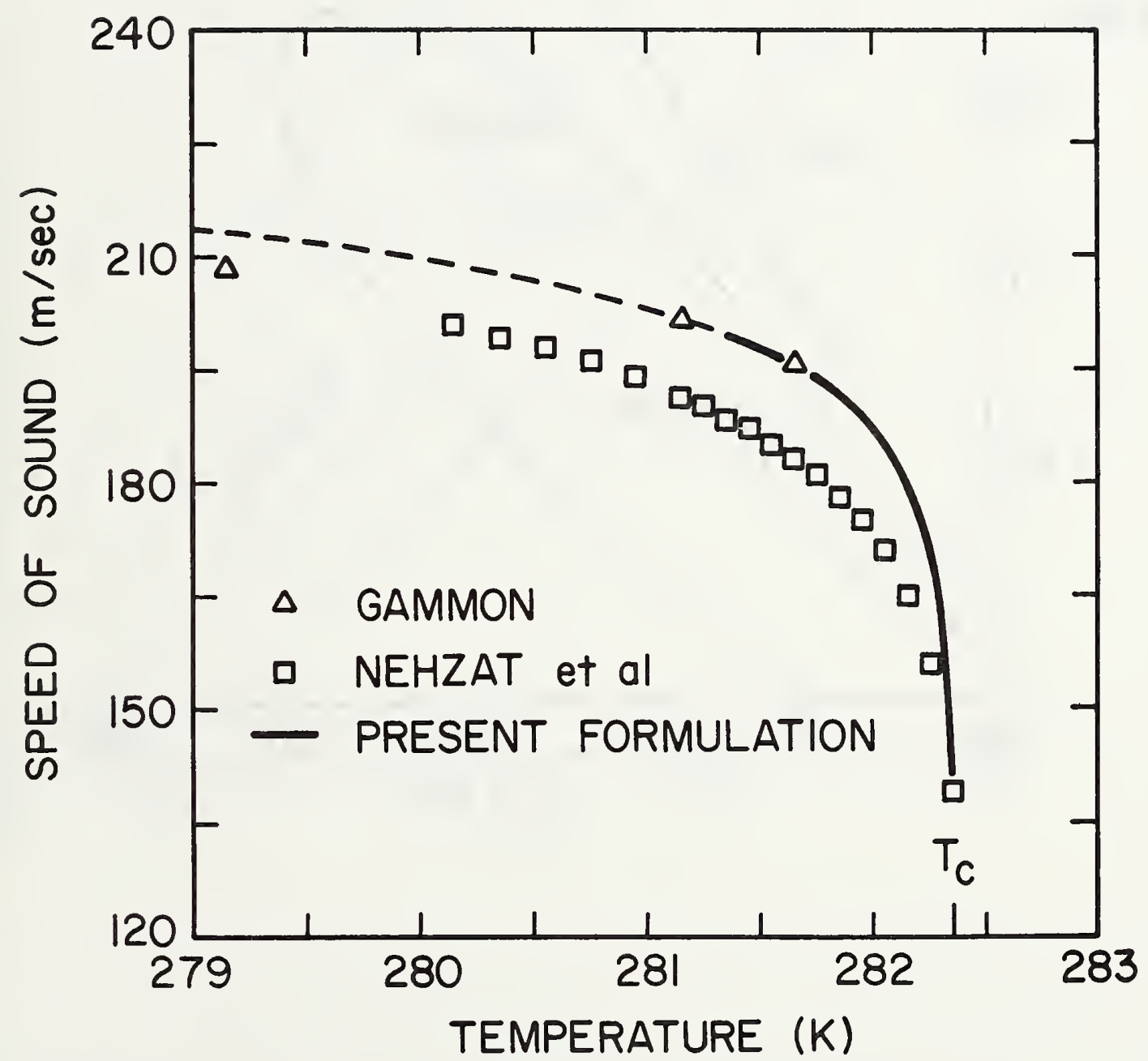

Fig. 13 Comparison of the formulation of Nehzat et a1. [23] and of our formulation with the experimental speed of sound data of Gammon for the saturated vapor below the critical temperature. The dashed part of the curve indicates our formulation when extrapolated outside its range of validity. 


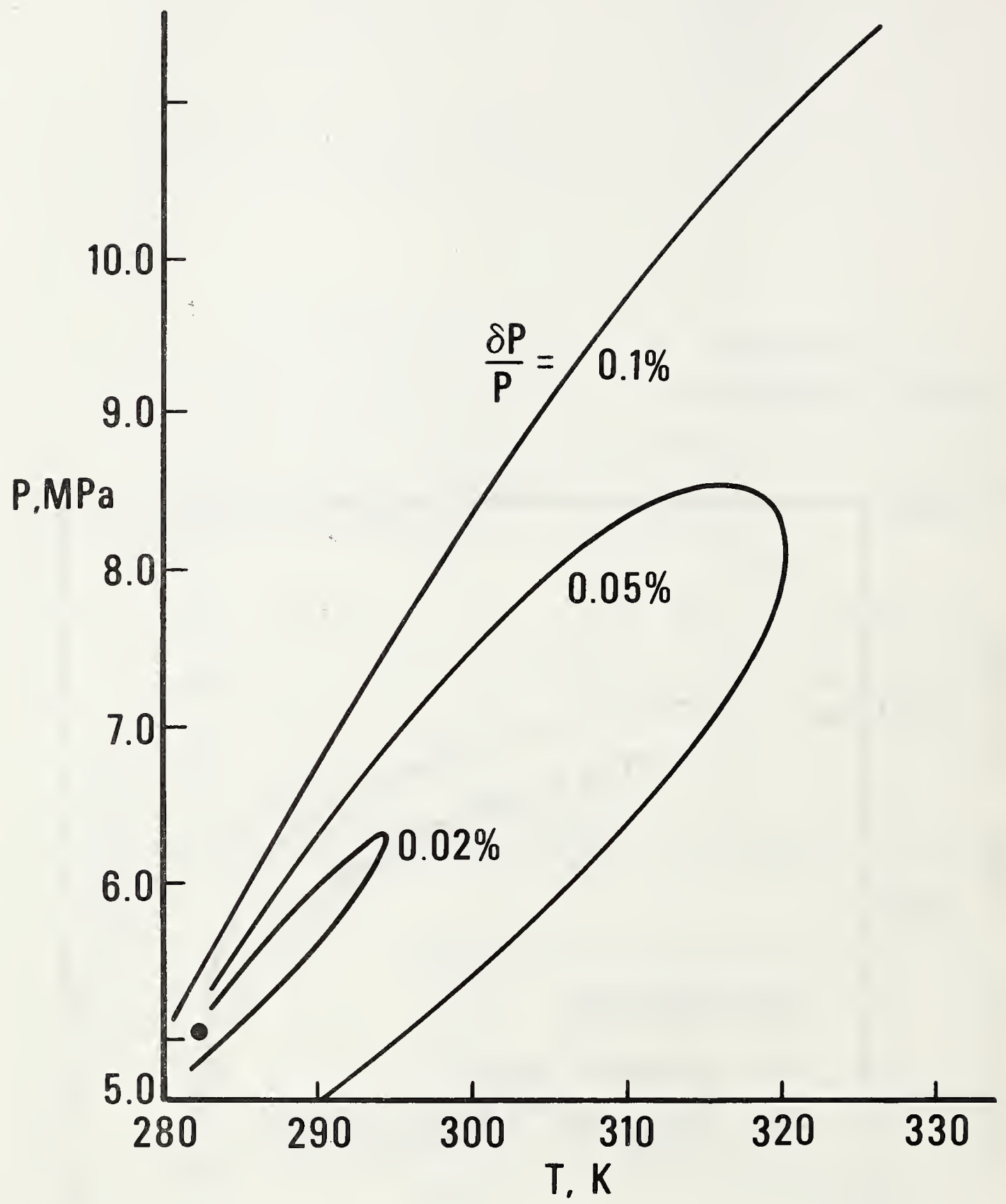

Fig. 14 The regions of P-T space that should be avoided in custody transfer if the pressure is measured to, respectively, $0.1 \%, 0.05 \%$ or $0.02 \%$ and an accuracy of $0.1 \%$ is desired in density. 


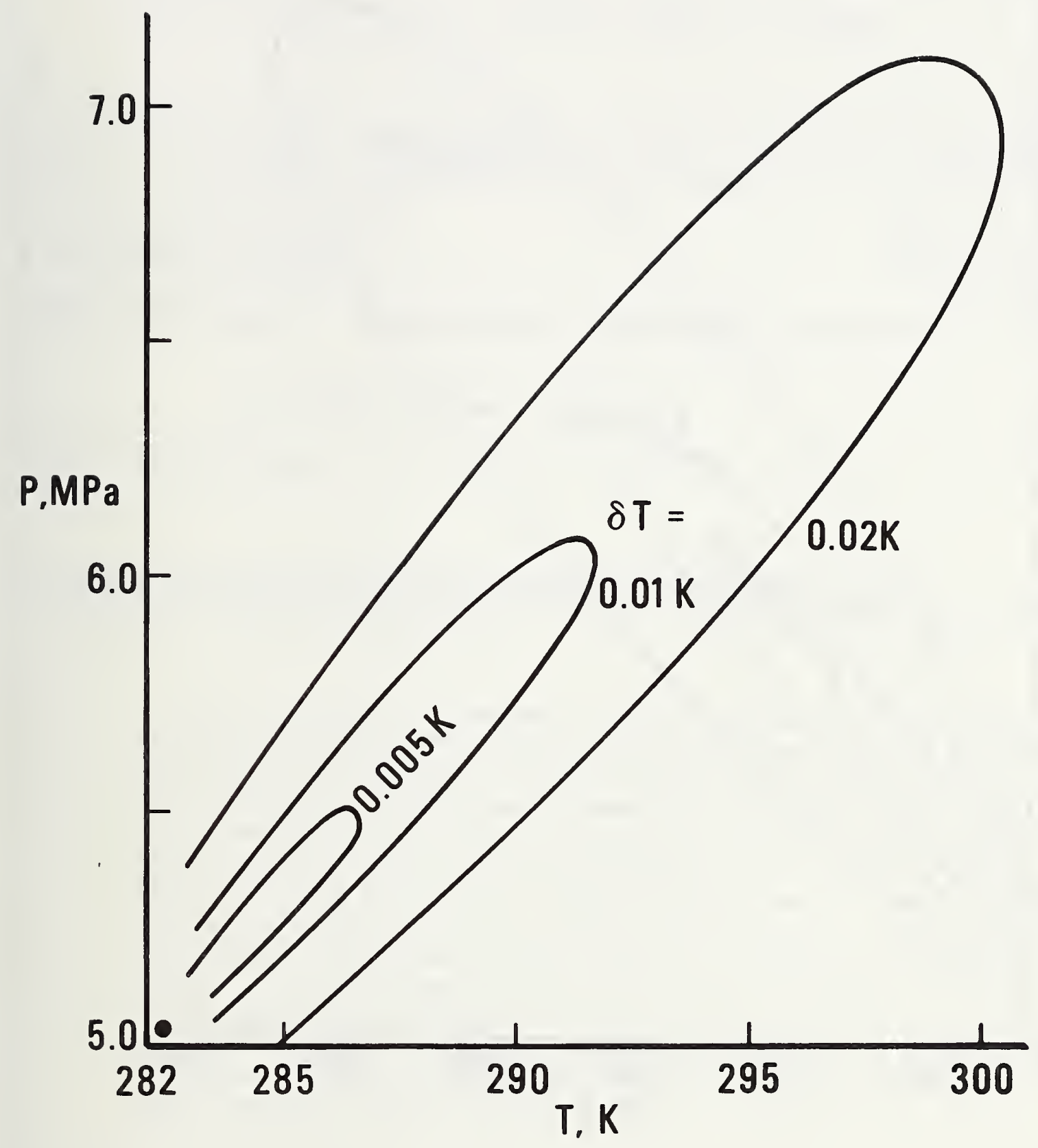

Fig. 15 The regions of P-T space that should be avoided in custody transfer if the temperature is measured to, respectively, $0.02 \mathrm{~K}, 0.01 \mathrm{~K}$ and $0.005 \mathrm{~K}$ and an accuracy of $0.1 \%$ is desired in density. 


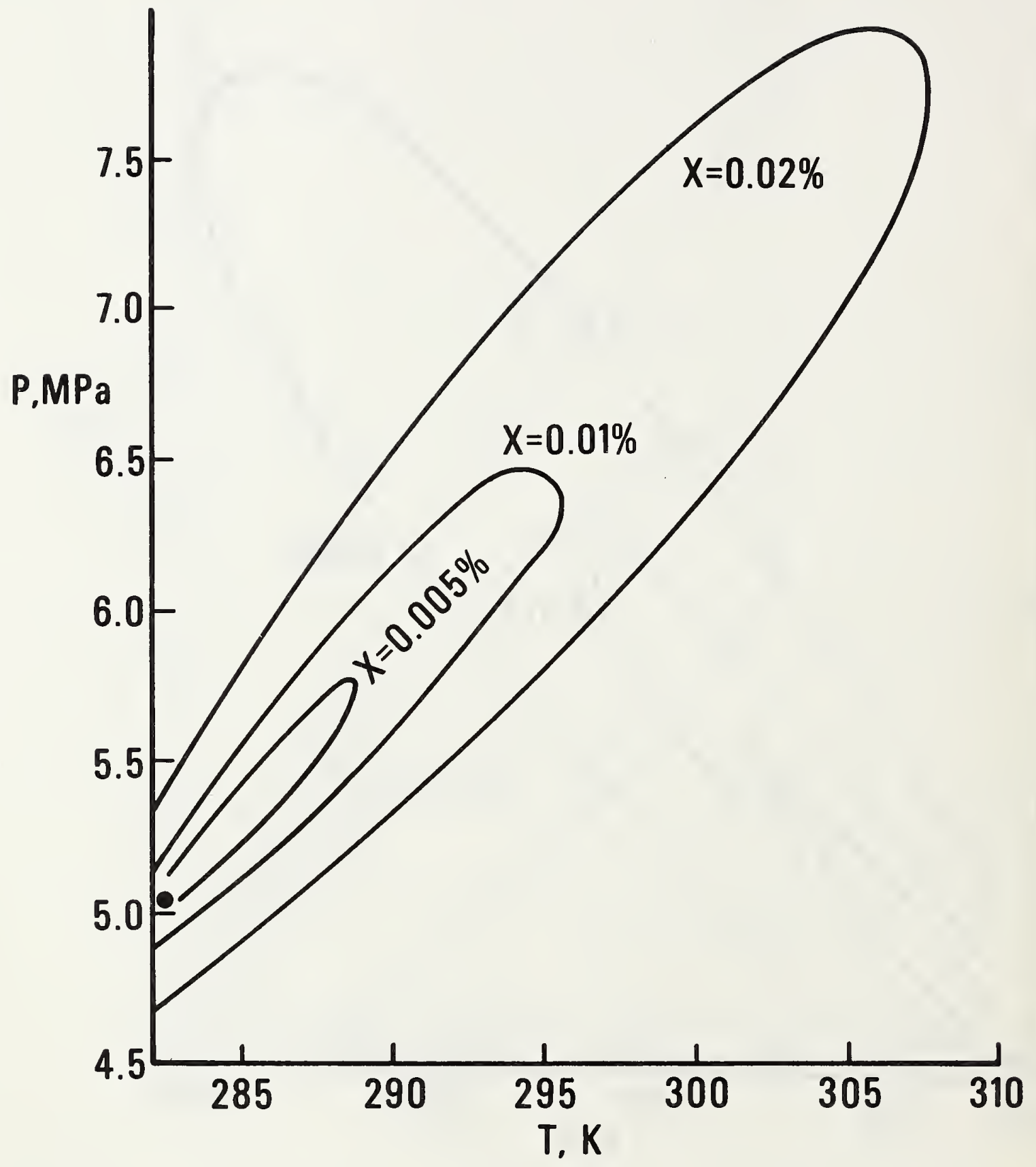

Fig. 16 The regions of P-T space that should be avoided in custody transfer if respectively $0.005 \%, 0.01 \%$ or $0.02 \%$ (in mole fraction) of an impurity very different in volatility from ethylene is present and an accuracy of $0.1 \%$ is desired in density. 
NBS-114A IREV. $2 \cdot 8 \mathrm{C}$ !

\begin{tabular}{|c|c|c|c|}
\hline U.S. DEPT. OF COMM. & 1. PUBLICATION OR & 2. Performing Organ. Report No & 3. Publication Date \\
BIBLIOGRAPHIC DATA & REPORT NO. & & May 1984 \\
SHEET (See instructions) & NBS TN 1189 & & \\
\hline
\end{tabular}

4. TITLE AND SUBTITLE

A Thermodynamic Surface for the Critical Region of Ethylene

5. $A U T H O R(S)$

J.M.H. Levelt Sengers, G.A. 01 chowy, B. Kamgar-Parsi, and J.V. Sengers

6. PERFORMING ORGANIZATION (If joint or other thon NBS, see instructions)

7. ContractGrant No.

MATIONAL BUREAU OF STANDARDS

DEPARTMENT OF COMMERCE

WASHINGTON, D.C. 20234

8. Type of Report \& Period Covered

Final

9. SPONSORING ORGANIZATION NAME AND COMPLETE ADDRESS (Street, City, Stote, ZIP)

See footnote on page 1.

10. SUPPLEMENTARY NOTES

Document describes a computer program; SF-185. FIPS Software Summary, is attached.

11. ABSTRACT (A 200-word or less factual summary of most significant information. If document includes a significant bibliogrophy or literature survey. mention it here)

Tables are presented of thermodynamic properties of ethylene in the range $279-300 \mathrm{~K}$ in temperature, $5.75-10.5 \mathrm{~mol} / \mathrm{dm}^{3}$ in density, which range includes the critical point. The tables presented here are based on the critical-point scaling laws and incorporate the critical anomalies as presently known from renormalization-group theory. The tables complement the formulation of the equation of state of fluid ethylene by McCarty and Jacobsen (NBS Tech. Note 1045, 1981) which does not claim accuracy near the critical point. The predictions of the present formulation are compared with four sets of recent PVT data, and with speed-of-sound and enthalpy data. Tables are presented of pressure, energy, enthalpy, entropy, specific heats and speed of sound as function of temperature along finely-spaced isochores. The computer program required for table generation is included. Even if the surface were perfect, the reliability of densities calculated at experimental pressures and temperatures of limited accuracy declines rapidly as the critical point is approached. Contour plots in P-T space are presented of regions to be avoided in custody transfer for given uncertainties in pressure, temperature and sample composition.

12. KEY WORDS (Six to twelve entries; alphabetical order; capitalize only proper names; and separate key words by semicolons) critical region; custody transfer; density; enthalpy; ethylene; equation of state; impurity; scaling laws; specific heat; speed of sound; supercritical extraction; thermodynamic properties

13. AVAILABILITY

Unlimited

$\square$ For Official Distribution. Do Not Release to NTIS

2 Order From Superintendent of Documents, U.S. Government Printing Office, Washington, D.C. 20402.

14. NO. OF PRINTED PAGES

\section{5}

15. Price

Order From National Technical Information Service (NTIS), Springfield, VA. 2216I 



NBS TECHNICAL NOTE 1189

A Thermodynamic Surface for the Critical Region of Ethylene May 1984

\section{Erratum}

Equation (A. 28), top p. 18, should read:

$$
w_{i}(\theta)=\left[\left(1-\alpha_{i}\right)\left(1-3 \theta^{2}\right) s_{i}(\theta)-\beta \delta\left(1-\theta^{2}\right) \theta s_{i}^{\prime}(\theta)\right] / q(\theta) .
$$





\section{NBS TECHNICAL PUBLICATIONS}

\section{PERIODICALS}

JOURNAL OF RESEARCH-The Journal of Research of the National Bureau of Standards reports NBS research and development in those disciplines of the physical and engineering sciences in which the Bureau is active. These include physics, chemistry, engineering, mathematics, and computer sciences. Papers cover a broad range of subjects, with major emphasis on measurement methodology and the basic technology underlying standardization. Also included from time to time are survey articles on topics closely related to the Bureau's technical and scientific programs. As a special service to subscribers each issue contains complete citations to all recent Bureau publications in both NBS and nonNBS media. Issued six times a year. Annual subscription: domestic \$18; foreign $\$ 22.50$. Single copy. $\$ 5.50$ domestic; $\$ 6.90$ foreign.

\section{NONPERIODICALS}

Monographs-Major contributions to the technical literature on various subjects related to the Bureau's scientific and technical activities.

Handbooks-Recommended codes of engineering and industrial practice (including safety codes) developed in couperation with interested industries, professional organizations, and regulatory bodies.

Special Publications-Include proceedings of conferences sponsored by NBS, NBS annual reports, and other special publications appropriate to this grouping such as wall charts, pocket cards, and bibliographies

Applied Mathematics Series-Mathematical tables, manuals, and studies of special interest to physicists, engineers, chemists, biologists, mathematicians, computer programmers, and others engaged in scientific and technical work

National Standard Reference Data Series-Provides quantitative data on the physical and chemical properties of materials, compiled from the world's literature and critically evaluated. Developed under a worldwide program coordinated by NBS under the authority of the National Standard Data Act (Public Law 90-396).

NOTE: The principal publication outlet for the foregoing data is the Journal of Physical and Chemical Reference Data (JPCRD) published quarterly for NBS by the American Chemical Society (ACS) and the American Institute of Physics (AIP). Suhscriptions, reprints, and supplements available from ACS, 1155 Sixteenth St., NW, Washington, DC 20056
Building Science Series-Disseminates technical irformation developed at the Bureau on building materials, components, systems, and whole structures. The series presents research results, test methods, and performance criteria related to the structural and environmental functions and the durability and safety characteristics of building elements and systems.

Technical Notes-Studies or reports which are complete in themselves but restrictive in their treatment of a subject. Analogous to monographs but not so comprehensive in scope or definitive in treatment of the subject area. Often serve as a vehicle for final reports of work performed at NBS under the sponsorship of other government agencies.

Voluntary Product Standards-Developed under procedures published by the Department of Commerce in Part 10. Title 15, of the Code of Federal Regulations. The standards establish nationally recognized requirements for products, and provide all concerned interests with a basis for common understanding of the characteristics of the products. NBS administers this program as a supplement to the activities of the private sector standardizing organizations

Consumer Information Series-Practical information, based on NBS research and experience, covering areas of interest to the consumer. Easily understandable language and illustrations provide useful background knowledge for shopping in today's technological marketplace.

Order the above NBS publications from: Superintendent of Documents. Government Printing Office, Washington, DC 20402.

Order the following NBS publications-FIPS and NBSIR's-from the National Technical Information Service, Springfield, VA 22161.

Federal Information Processing Standards Publications (FIPS PUB)-Publications in this series collectively constitute the Federal Information Processing Standards Register. The Register serves as the official source of information in the Federal Government regarding standards issued by NBS pursuant to the Federal Property and Administrative Services Act of 1949 as amended, Public Law 89-306 (79 Stat. 1127), and as implemented by Executive Order 11717 (38 FR 12315, dated May 11, 1973) and Part 6 of Title 15 CFR (Code of Federal Regulations).

NBS Interagency Reports (NBSIR)-A special series of interim or final reports on work performed by NBS for outside sponsors (both government and non-government). In general, initial distribution is handled by the sponsor: public distribution is by the National Technical Information Service, Springfield, VA 22161, in paper copy or microfiche form. 
U.S. Department of Commerce

National Bureau of Standards

Washington, D.C. 20234

Official Business

Penalty for Private Use $\$ 300$ 